\title{
ENTROPY THEORY FOR THE PARAMETRIZATION OF THE EQUILIBRIUM STATES OF PIMSNER ALGEBRAS
}

\author{
EVGENIOS T.A. KAKARIADIS
}

\begin{abstract}
We consider Pimsner algebras that arise from $\mathrm{C}^{*}$-correspondences of finite rank, as dynamical systems with their rotational action. We revisit the Laca-Neshveyev classification of their equilibrium states at positive inverse temperature along with the parametrizations of the finite and the infinite parts simplices by tracial states on the diagonal. The finite rank entails an entropy theory that shapes the KMS-structure. We prove that the infimum of the tracial entropies dictates the critical inverse temperature, below which there are no equilibrium states for all Pimsner algebras. We view the latter as the entropy of the ambient $\mathrm{C}^{*}$-correspondence. This may differ from what we call strong entropy, above which there are no equilibrium states of infinite type. In particular, when the diagonal is abelian then the strong entropy is a maximum critical temperature for those. In this sense we complete the parametrization method of Laca-Raeburn and unify a number of examples in the literature.
\end{abstract}

\section{INTRODUCTION}

The Fock space construction gives a concrete quantization of systems in terms of Hilbertian operators. Originating from Quantum Mechanics, it has seen an important generalization to Hilbert bimodules over $\mathrm{C}^{*}$-algebras, better known as $\mathrm{C}^{*}$-correspondences. The key element is the existence of a $\mathrm{C}^{*}$-algebra $A$ acting "externally" on $X$ and of an $A$-valued inner product. Rieffel [35] originally envisioned $\mathrm{C}^{*}$-correspondences as a tool to identify $\mathrm{C}^{*}$-algebras in terms of their representation theory. Pimsner [33] much later extended the theory to accommodate a range of examples of Operator Algebras arising from $\mathrm{C}^{*}$-dynamics and graphs. The Pimsner algebras generalize the well known Toeplitz- and Cuntz-algebras and they have been under considerable study since their introduction. By now they form a topic in its own respect with several interactions with graph theory and ring theory. The $\mathrm{C}^{*}$-correspondence machinery is now viewed as an effective way for quantizing geometric structures that evolve in discrete time.

Nevertheless, the interplay of $\mathrm{C}^{*}$-algebras with Quantum Statistical Mechanics goes well beyond that point. Taking motivation from ideal gases, there is an analogue of a Kubo-MartinSchwinger condition for states of $\mathrm{C}^{*}$-algebras that admit an $\mathbb{R}$-action, even when moving beyond the trace class operators. See for example the seminal monographs of Bratelli-Robinson [3, 4]. The parametrization of equilibrium states has been an essential task in the past 30 years, as they can serve as an invariant for $\mathbb{T}$-equivariant isomorphisms. To give only but a fragment of a very long list we mention the Cuntz-algebra $[\mathbf{1 0}, \mathbf{3 1}], \mathrm{C}^{*}$-algebras of different types of dynamical systems $[2,11,13,16,20,22,26,27,34,37,38]$, graph $C^{*}$-algebras $[14,15,18,36], C^{*}$-algebras related to number systems $[\mathbf{1}, \mathbf{7}, \mathbf{2 4}, \mathbf{2 5}]$ and to subshifts $[\mathbf{9}, \mathbf{2 9}]$, and Pimsner algebras [17, 23].

The major steps for classifying the equilibrium states of Pismner algebras were established in the seminal paper of Laca and Neshveyev [23]. Their arguments were further refined by Laca and Raeburn [25] in their study of $\mathrm{C}^{*}$-algebras arising from number systems. The approach of Laca-Raeburn has been very influential, and effectively applicable in a big variety of examples, e.g. $[\mathbf{1 4}, \mathbf{1 5}, \mathbf{2 0}, \mathbf{2 6}, \mathbf{2 7}]$. However in each occasion ad-hoc data is used to trigger the algorithm. The aim of this paper is to show how these ideas combine with the notion of entropy of Pinzari, Watatani and Yotetani [34] that is induced when the ambient $\mathrm{C}^{*}$-correspondences have finite rank; an assumption that holds in the aforementioned cases. The KMS-structure of the Pimsner algebras in $[14,15,18,20,26,27]$ follows as an application of this analysis.

2010 Mathematics Subject Classification. 46L30, 46L55, 46L08, 58 B34.

Key words and phrases: KMS-condition, equilibrium states, $\mathrm{C}^{*}$-correspondences, Pimsner algebras. 
1.1. Motivation. The Toeplitz-Pimsner algebra $\mathcal{T}_{X}$ is the $\mathrm{C}^{*}$-algebra generated by the left creation operators of $X$ and $A$ acting on the Fock space $\mathcal{F} X$. In addition, there is a range of Pimsner algebras that encodes desirable redundancies. Every quotient of $\mathcal{T}_{X}$ by $\mathbb{T}$-equivariant relations gives rise to a $J$-relative Cuntz-Pimsner algebra $\mathcal{O}(J, X)$, where $J \subseteq \phi_{X}^{-1}(\mathcal{K} X)$ for the left action $\phi_{X}$ of $A$ and the compact operators $\mathcal{K} X$. Among those the Cuntz-Pimsner algebra $\mathcal{O}_{X}$ is of central importance and arises when $J$ is Katsura's ideal [21]. It is the smallest $\mathbb{T}$-equivariant quotient of $\mathcal{T}_{X}$ that admits a faithful copy of $A$ and $X$ [19]. In general $\mathcal{O}_{X} \neq \mathcal{O}\left(\phi_{X}^{-1}(\mathcal{K} X), X\right)$ but they coincide with $\mathcal{O}(A, X)$ when $X$ is injective and $\phi_{X}(A) \subseteq \mathcal{K} X$.

Laca and Neshveyev [23] studied actions implemented by one-parameter unitaries for injective $\mathrm{C}^{*}$-correspondences. Their main tool was the use of induced traces from $[\mathbf{6}, \mathbf{8}, \mathbf{3 2}]$. In this way they were able to classify the equilibrium states of $\mathcal{T}_{X}$ in terms of their restrictions on the diagonal by using iterations of the inducing map at each level of the Fock space. Following Exel-Laca [13], they proved a Wold decomposition into a finite part (given by a series of iterations of a tracial state on the diagonal) and an infinite part (where iterations are stable). They showed that $\mathcal{T}_{X}$ admits a rich KMS-structure from which they could derive that of $\mathcal{O}_{X}$ (when $X$ is injective) through the infinite part. A characterization was also given for ground states.

Later Laca-Raeburn [25] refined the main tools of [23] for a specific class of Pimsner algebras coming from number systems. From then on the interest was restricted to dynamics implemented by the rotational action. Most notably they use the statistical approximations of [23] to parameterize the finite part by tracial states on $A$. As we shall explain later there is a difference between the parametrizations in [23] and in [25]. Likewise, weak*-homeomorphic parametrizations were given for both ground states and $\mathrm{KMS}_{\infty}$-states in [25].

In turn, a number of subsequent works, e.g. $[\mathbf{1 4}, \mathbf{1 5}, \mathbf{2 0}, \mathbf{2 6}, \mathbf{2 7}]$, were greatly influenced by the parametrizations of $[\mathbf{2 3}, \mathbf{2 5}]$ and applied their method to other examples of Pimsner algebras. A re-appearing theme is the existence of two critical temperatures $\beta_{c} \geq \beta_{c}^{\prime}$ for which:

(a) for $\beta>\beta_{c}$ the algorithm of [25] gives all equilibrium states for $\mathcal{T}_{X}$;

(b) for $\beta=\beta_{c}^{\prime}$ there is an association with averaging states; and

(c) there are no equilibrium states below $\beta_{c}^{\prime}$.

At the other extreme $\mathcal{O}(A, X)$ is not amenable to the construction of (a) but it provides the states for (b). Such an example is the averaging state on the Cuntz-algebra $\mathcal{O}_{d}$ which is the only possible equilibrium state (and it appears at $\beta=\log d$ ).

The critical temperatures often coincide and can be associated to structural data of the original construct. For example, an Huef-Laca-Raeburn-Sims [14] show that $\beta_{c}=\beta_{c}^{\prime}$ is the logarithm of the Perron-Fröbenius eigenvalue when the graph is irreducible. In a continuation [15] the authors also show that a more rich structure appears for general graphs. That was also verified by KajiwaraWatatani [18] who studied the KMS-structure of Cuntz-Krieger $\mathrm{C}^{*}$-algebras. In the process they achieve also a parametrization of the infinite part of $\mathcal{O}(J, X)$ for $J$ inside Katsura's ideal under some assumptions on the $\mathrm{C}^{*}$-correspondence. However this does not cover the infinite part in the non-injective case, i.e., it does not cover the case $J=\phi_{X}^{-1}(\mathcal{K} X)$. These works motivate the following question:

Q. How $A$ and $X$ dictate the critical temperature(s) beyond which we don't have equilibrium states of Pimsner algebras?

In the current paper we show how this is done under the assumption that $X$ attains a finite set $\left\{x_{1}, \ldots, x_{d}\right\}$ of vectors in its unit ball such that $1_{X}=\sum_{i \in[d]} \theta_{x_{i}, x_{i}}$. Equivalently, when the adjointable operators of $X$ are compact. This is satisfied in the aforementioned examples, and sometimes on the much stronger side of the vectors being orthogonal. We are not assuming orthogonality here.

Also, we mention that we consider just the dynamics coming from the rotational $\mathbb{T}$-action for which there is a physical interpretation. Recall that the starting point for Gibbs states is the action implemented by $r \mapsto e^{i r(H-\kappa N)}$, where $H$ is the Hamiltonian, $N$ is the number operator and $\kappa$ is the chemical potential. When $H$ is the Hamiltonian of a Quantum Harmonic Oscillator then it admits the solution $H=h \omega(1 / 2+N)$ for the energy dimension $h \omega / 2$ of the ground state, and 
the action is implemented by $r \mapsto e^{i r h \omega / 2} e^{i r(h \omega-\kappa) N}$. Since $N$ is unbounded some effort is required to make the action precise. This can be seen for example in Proposition 2.2 where it is shown that $r \mapsto \gamma_{e^{i r s}}$ realizes any action implemented by $r \mapsto e^{i r(c+s N)}$ for $c \in \mathbb{C}$ and $s \in \mathbb{R}$. In what follows we make the normalization $h \omega-\kappa=1$. Recall that $\kappa<h \omega / 2$ for any Quantum Harmonic Oscillator and thus substituting $\beta$ by $(h \omega-\kappa) \beta$ covers all cases.

1.2. Decomposition and parametrization. We write $\mathrm{E}_{\beta}(\mathcal{O}(J, X))$ for the $(\sigma, \beta)$-KMS states of the $J$-relative Cuntz-Pimsner algebra $\mathcal{O}(J, X)$ with respect to the action $r \mapsto \sigma_{r}:=\gamma_{e^{i r}}$. Every $\mathcal{O}(J, X)$ is the quotient of $\mathcal{T}_{X}$ by a $\mathbb{T}$-equivariant ideal and hence in order to understand $\mathrm{E}_{\beta}(\mathcal{O}(J, X))$ it suffices to do so for $\mathrm{E}_{\beta}\left(\mathcal{T}_{X}\right)$. We need to revisit in detail the main points of [18, 23] and in particular see how the method of $[\mathbf{2 5}]$ extends to unify $[\mathbf{1 4}, \mathbf{2 0}, \mathbf{2 6}, \mathbf{2 7}]$.

In what follows fix $\left\{x_{1}, \ldots, x_{d}\right\}$ be a finite unit decomposition. Then $\left\{x_{\mu}|| \mu \mid=n\right\}$ yields a unit decomposition for $X^{\otimes n}$, where we write $x_{\mu_{n} \cdots \mu_{1}}=x_{\mu_{n}} \otimes \cdots \otimes x_{\mu_{1}}$ for a word $\mu=\mu_{n} \cdots \mu_{1}$ on the $d$ symbols. Consequently the projections $p_{n}: \mathcal{F} X \rightarrow X^{\otimes n}$ and the compacts $\mathcal{K}(\mathcal{F} X)$ are in $\mathcal{T}_{X}$. The finite and the infinite parts of the Wold decomposition at $\beta>0$ of [23] form respectively the convex sets:

$$
\mathrm{E}_{\beta}^{\mathrm{fin}}\left(\mathcal{T}_{X}\right):=\left\{\varphi \in \mathrm{E}_{\beta}\left(\mathcal{T}_{X}\right) \mid \sum_{k=0}^{\infty} \varphi\left(p_{k}\right)=1\right\} \quad \text { and } \quad \mathrm{E}_{\beta}^{\infty}\left(\mathcal{T}_{X}\right):=\left\{\varphi \in \mathrm{E}_{\beta}\left(\mathcal{T}_{X}\right) \mid \varphi\left(p_{0}\right)=0\right\} .
$$

In particular $\mathrm{E}_{\beta}^{\infty}\left(\mathcal{T}_{X}\right)$ corresponds to the states annihilating $\mathcal{K}(\mathcal{F} X)$ (and thus to those that factor through $\mathcal{O}(A, X)$ ), and $\mathrm{E}_{\beta}^{\mathrm{fin}}\left(\mathcal{T}_{X}\right)$ corresponds to those that restrict to states on $\mathcal{K}(\mathcal{F} X)$ (see Theorem 4.6). We then construct the parametrization of each convex set by a specific convex set in the tracial states $\mathrm{T}(A)$ of $A$. This is linked to the formal series

$$
c_{\tau, \beta}:=\sum_{k=0} e^{-k \beta} \sum_{|\mu|=k} \tau\left(\left\langle x_{\mu}, x_{\mu}\right\rangle\right) \text { for } \tau \in \mathrm{T}(A) \text { and } \beta>0 .
$$

We thus need to consider the sets that arise from two extreme cases:

$$
\mathrm{T}_{\beta}(A):=\left\{\tau \in \mathrm{T}(A) \mid c_{\tau, \beta}<\infty\right\} \text { and } \operatorname{AVT}_{\beta}(A):=\left\{\tau \in \mathrm{T}(A) \mid e^{\beta} \tau(\cdot)=\sum_{i \in[d]} \tau\left(\left\langle x_{i}, \cdot x_{i}\right\rangle\right)\right\} .
$$

Notice that $c_{\tau, \beta}=\sum_{k=0}^{\infty} 1$ for every $\tau \in \operatorname{AVT}_{\beta}(A)$.

The parametrization of $\mathrm{E}_{\beta}^{\mathrm{fin}}\left(\mathcal{T}_{X}\right)$ is constructive and follows from $[\mathbf{2 3}, \mathbf{2 5}]$. In Theorem 6.1 we show that there is a bijection

$$
\Phi: \mathrm{T}_{\beta}(A) \rightarrow \mathrm{E}_{\beta}^{\mathrm{fin}}\left(\mathcal{T}_{X}\right) \text { such that } \Phi(\tau)\left(p_{0}\right)=c_{\tau, \beta}^{-1} .
$$

In particular $\Phi$ can be reconstructed by

$$
\Phi(\tau)\left(t\left(\xi^{\otimes n}\right) t\left(\xi^{\otimes m}\right)^{*}\right)=\delta_{n, m} c_{\tau, \beta} \sum_{k=0}^{\infty} e^{-(k+n) \beta} \sum_{|\mu|=k} \tau\left(\left\langle\eta^{\otimes m} \otimes x_{\mu}, \xi^{\otimes n} \otimes x_{\mu}\right\rangle\right)
$$

for all $\xi^{\otimes n} \in X^{\otimes n}$ and $\eta^{\otimes m} \in X^{\otimes m}$. When $\mathrm{E}_{\beta}^{\text {fin }}\left(\mathcal{T}_{X}\right)$ is weak*-closed then $\Phi$ is a weak*homeomorphism. As a new outcome of this analysis we derive that the map $\Phi$ preserves convex combinations (by weighting over the $c_{\tau, \beta}$ ), and thus it preserves the extreme points.

Theorem 6.1 uses the crux of the arguments of [23, proof of Theorem 2.1] but as with $[\mathbf{1 4}, \mathbf{1 5}$, $\mathbf{2 0}, \mathbf{2 5}, \mathbf{2 6}, \mathbf{2 7}]$ there are slight differences. First of all the correspondence between $\mathrm{E}_{\beta}\left(\mathcal{T}_{X}\right)$ and a subset of $\mathrm{T}(A)$ is provided in [23, Theorem 2.1], yet as a correspondence $\mathrm{E}_{\beta}\left(\mathcal{T}_{X}\right) \rightarrow \mathrm{T}(A)$ given by restriction $\left.\Phi \mapsto \Phi\right|_{A}$, and it is not linked to $\mathrm{T}_{\beta}(A)$. In the comments preceding [23, Definition 2.3 ] it is hinted how a $\tau$ might be obtained from $\Phi$ but the suggested map requires normalization (by the possibly non-constant $c_{\tau, \beta}$ ). The constructive approach we take here tackles this point. Secondly, $\Phi$ is obtained through induced representations of Toeplitz-Pimsner algebras rather than the theory of induced traces from $[\mathbf{6}, \mathbf{8}, \mathbf{3 2}]$.

The infinite part is dealt with in Theorem 7.1 where an affine weak*-homeomorphism is constructed:

$$
\Psi:\left\{\tau \in \operatorname{AVT}_{\beta}(A)|\tau|_{I}=0\right\} \rightarrow \mathrm{E}_{\beta}^{\infty}\left(\mathcal{T}_{X}\right) \text { such that }\left.\Psi(\varphi)\right|_{A}=\tau,
$$


for the ideal of $A$

$$
I:=\left\{a \in A \mid \lim _{n}\left\|\phi_{X}(a) \otimes \operatorname{id}_{X}^{\otimes n-1}\right\|=0\right\} .
$$

The ideal $I$ is the kernel of the canonical quotient $q: \mathcal{T}_{X} \rightarrow \mathcal{O}(A, X)$ and arises from the fact that every $\varphi \in \mathrm{E}_{\beta}^{\infty}\left(\mathcal{T}_{X}\right)$ factors through $q$. The proof follows the lines of [18, Theorem 3.18] with the additional use of $I$. The main tool is that the fixed point algebra is the inductive limit of the $\mathcal{K} X^{\otimes n}$ when $X$ is injective. It has been implicitly applied in $[\mathbf{2 9}, \mathbf{2 0}]$ to obtain equilibrium states at the critical temperature.

The affine weak*-homeomorphism has been obtained in [23, Theorem 2.1 and Theorem 2.5], when $X$ is injective and non-degenerate, but with an entirely different line of attack. At the end of [23, proof of Theorem 2.1] it is shown that any equilibrium state can be given as a limit of finite type states on $\sigma^{\varepsilon}$ perturbed actions so that $\lim _{\varepsilon \rightarrow 0} \sigma^{\varepsilon}=\sigma$. Hence they verify that [23, Formula (2.2)] gives a well defined extension of a state from $A$ to $\mathcal{T}_{X}$. Then [23, Theorem 2.5] asserts that [23, Formula (2.2)] gives a state $\varphi$ of infinite type if and only if $\left.\varphi\right|_{A} \in \operatorname{AVT}_{\beta}(A)$. Theorem 7.1 on the other hand constructs directly the extension within the same action $\sigma$ without any conditions on $X$. Moreover this method applies to parametrize the gauge-invariant tracial states on $\mathcal{O}(A, X)$.

By passing to a $\mathbb{T}$-equivariant quotient we derive a similar characterization for any $J$-relative Cuntz-Pimsner algebra $\mathcal{O}(J, X)$ through the following scheme:

$$
\begin{array}{cc}
\mathrm{T}_{\beta}(A) \cap\left\{\tau \in \mathrm{T}(A)|\tau|_{J}=0\right\} \longrightarrow & \Phi \\
\operatorname{AVT}_{\beta}(A) \cap\left\{\tau \in \mathrm{T}(A)|\tau|_{I}=0\right\} \longrightarrow & \oplus_{\text {-convex }}^{\mathrm{fin}}(\mathcal{O}(J, X)) \\
& =\mathrm{E}_{\beta}^{\infty}(\mathcal{O}(J, X)) .
\end{array}
$$

Figure. Parametrization of equilibrium states of $\mathcal{O}(J, X)$.

Of course this has to be taken with care as it may be that $\mathrm{E}_{\beta}^{\infty}(\mathcal{O}(J, X))$ or $\mathrm{E}_{\beta}^{\text {fin }}(\mathcal{O}(J, X))$ is empty for some choices of $\beta$ and $J$. This brings us to the main point of the discussion that captured our interest in the first place.

1.3. Entropy. Taking motivation from the classical case, entropy has been used in various guises. See the excellent monograph of Neshveyev-Størmer in this respect [30]. Our approach is closer to that of Pinzari-Watatani-Yotetani [34] who considered imprimitivity bimodules with finite left and right unit decompositions. The starting point is that the statistical approximation (1.5) works only when $c_{\tau, \beta}<\infty$. The ratio test may not be conclusive for all formal series $c_{\tau, \beta}$ but it can be used to define the following notions of entropies ${ }^{1}$. The entropy of a tracial state $\tau$ of $A$ is given by

$$
h_{X}^{\tau}:=\limsup _{k} \frac{1}{k} \log \sum_{|\mu|=k} \tau\left(\left\langle x_{\mu}, x_{\mu}\right\rangle\right) .
$$

Notice that $h_{X}^{\tau} \leq \beta$ if $\tau \in \mathrm{T}_{\beta}(A)$, and that $h_{X}^{\tau}=\beta$ if $\tau \in \operatorname{AVT}_{\beta}(A)$. Moreover $h_{X}^{\tau}$ is independent of the choice of the unit decomposition. On the other hand for a fixed unit decomposition $x=$ $\left\{x_{1}, \ldots, x_{d}\right\}$ we can define

$$
h_{X}^{x}:=\limsup _{k} \frac{1}{k} \log \left\|\sum_{|\mu|=k}\left\langle x_{\mu}, x_{\mu}\right\rangle\right\|_{A}
$$

where the limsup is actually a limit. The strong entropy of $X$ is then given by

$$
h_{X}^{s}:=\inf \left\{h_{X}^{x} \mid x=\left\{x_{1}, \ldots, x_{d}\right\} \text { is a unit decomposition for } X\right\} .
$$

If $A$ is abelian then $h_{X}^{s}$ is the same for all unit decompositions. We define the entropy of $X$ as the critical temperature below which we do not attain equilibrium states for any Pimsner algebra, i.e.,

$$
h_{X}:=\inf \left\{\beta>0 \mid \mathrm{E}_{\beta}\left(\mathcal{T}_{X}\right) \neq \emptyset\right\} \quad(\text { with } \inf \emptyset:=\infty) .
$$

\footnotetext{
${ }^{1}$ As we are concerned about convergence of series we will make the convention that $\lim \sup _{k} k^{-1} \log a_{k}=0$ if $a_{k}=0$ eventually.
} 
By weak*-compactness the infimum is actually a minimum. In Proposition 5.7, Corollary 6.3, Proposition 7.2 and Corollary 7.3 we show that:

(i) $h_{X}^{\tau} \leq h_{X}^{s} \leq \log d$ for every $\tau \in \mathrm{T}(A)$.

(ii) If $\beta>h_{X}^{s}$ then $\mathrm{T}_{\beta}(A)=\mathrm{T}(A)$ and thus $\mathrm{E}_{\beta}^{\infty}\left(\mathcal{T}_{X}\right)=\emptyset$ and $\mathrm{E}_{\beta}\left(\mathcal{T}_{X}\right)=\mathrm{E}_{\beta}^{\mathrm{fin}}\left(\mathcal{T}_{X}\right)$.

An essential application of [34] gives also that $\mathrm{E}_{h_{X}^{s}}^{\infty}\left(\mathcal{T}_{X}\right) \neq \emptyset$ when $A$ is abelian and $h_{X}^{s}>0$. In Corollary 7.3 we provide one of the main conclusions of this analysis; namely, that the entropy of $X$ can be recovered from the state entropies in the following way:

$$
h_{X}=\max \left\{0, \inf \left\{h_{X}^{\tau} \mid \tau \in \mathrm{T}(A)\right\}\right\} .
$$

In fact, if $h_{X}>0$ or if $h_{\tau} \geq 0$ for all $\tau \in \mathrm{T}(A)$ then $h_{X}=\min \left\{h_{X}^{\tau} \mid \tau \in \mathrm{T}(A)\right\}$. Consequently, if $h_{X}^{\tau}=h_{X}^{s}$ for all $\tau \in \mathrm{T}(A)$ then $\mathrm{E}_{\beta}^{\infty}\left(\mathcal{T}_{X}\right)=\emptyset$ whenever $\beta>h_{X}$. This gives the KMS-states theory of $\mathcal{O}_{d}$ in a nutshell, and reflects what is known for specific cases in the literature. In Section 8 we emphasize by examples that:

(iii) The infimum over all $h_{X}^{x}$ is required in the definition of $h_{X}^{s}$, as the notion of an orthonormal basis is not well defined for Hilbert modules over non-abelian $\mathrm{C}^{*}$-algebras.

(iv) If $A$ is abelian and $X$ attains an orthonormal basis then $h_{X}^{\tau}=h_{X}=h_{X}^{s}=\log d$ for all $\tau \in$ $\mathrm{T}(A)$. Moreover $\mathrm{E}_{\beta}\left(\mathcal{T}_{X}\right)=\mathrm{E}_{\beta}^{\mathrm{fin}}\left(\mathcal{T}_{X}\right) \neq \emptyset$ for all $\beta>\log d$, and $\mathrm{E}_{\log d}\left(\mathcal{T}_{X}\right)=\mathrm{E}_{\log d}^{\infty}\left(\mathcal{T}_{X}\right) \neq \emptyset$.

(v) There may be both finite and infinite parts for $\mathcal{T}_{X}$ when $\beta \in\left(h_{X}, h_{X}^{s}\right)$.

As a second application we show how the entropy theory fully recovers the KMS-structure of Pimsner algebras of irreducible graphs $[\mathbf{1 4}, \mathbf{1 8}]$, and that of Pimsner algebras related to dynamical systems or self-similar actions of $[\mathbf{2 0}, \mathbf{2 6}, \mathbf{2 7}]$. For these examples we derive item (v) above, where the value $d$ is specified by the intrinsic data of the related $\mathrm{C}^{*}$-correspondence. We also take a look at the KMS-simplices for reducible graphs that have been identified in [15]. The main tool there is to study hereditary closures of connected components and quotients by passing to subgraphs. As a third application we show how the theory of [15] is recovered just by using entropies, and let us provide a description here.

Let $G_{1}, \ldots, G_{m}$ be the irreducible components of $G$ with respect to which $G$ takes up an upper triangular form ${ }^{2}$. Each component comes with its Perron-Fröbenius eigenvalue $\lambda_{G_{s}}$ and it is well known that

$$
h_{G}:=\limsup _{k} \frac{1}{k} \log \left(\sum_{i, j \in G}\left(G^{k}\right)_{i j}\right)=\max \left\{\log \lambda_{G_{1}}, \ldots, \log \lambda_{G_{m}}\right\}
$$

for the entropy $h_{G}$ of the graph. The first step is to identify $h_{X}$ and $h_{X}^{s}$ (Theorem 8.9). We verify that $h_{G}$ equals the strong entropy of the graph $\mathrm{C}^{*}$-correspondence. Next we say that an irreducible component $G_{s}$ is a sink if there are no paths emitting from $G_{s}$ that end at a vertex outside $G_{s}$. If there exists a zero sink component then $h_{X}=0$; otherwise

$$
h_{X}=\min \left\{\log \lambda_{G_{s}} \mid G_{s} \text { is a non-zero sink irreducible component of } G\right\} .
$$

A direct computation shows that the averaging traces correspond exactly to positive eigenvectors of the transpose matrix $G^{t}$. In Proposition 8.6 it is shown en passant that

$$
h_{X}^{\tau}=\max \left\{\log \lambda_{G_{s}} \mid G_{s} \text { is communicated by some } v_{r} \text { in the support of } \tau\right\} \text {, }
$$

and that $\tau \in \mathrm{T}_{\beta}(A)$ if and only if $h_{X}^{\tau}<\beta$. The classification of the KMS-simplices of [15] then follows in the following way (Theorem 8.13). There are phase transitions exactly at the numbers

$$
\Lambda:=\left\{\log \lambda_{G_{s}} \mid \lambda_{G_{s}} \geq \lambda_{G_{r}} \text { whenever } G_{r} \text { is communicated by } G_{s}\right\} .
$$

The $G_{s}$ that contribute to $\Lambda$ are called $\lambda_{G_{s}}$-maximal, and correspond to the minimal components described in [15]. For convenience we order $\Lambda$ by

$$
h_{X}=\log \lambda_{1}<\cdots<\log \lambda_{q}=h_{X}^{s},
$$

\footnotetext{
${ }^{2}$ We write $G_{i j}$ for the edges from $i$ to $j$. Although this is the transpose of [15], it facilitates some computations in the proofs.
} 
so that for every $n$ there are $G_{n, 1}, \ldots, G_{n, k_{n}}$ that are $\lambda_{n}$-maximal. Let us set

$$
V_{n}:=\left\{v \in G \mid v \text { communicates with some } G_{n, s}, s=1, \ldots, k_{n}\right\} .
$$

Since $h_{X}^{\tau}>\log \lambda_{n}$ if and only if the support of $\tau$ communicates with $G_{j, s}$ for some $j>n$ we get

$$
\mathrm{T}_{\beta}(A)=\left\{\tau \in \mathrm{T}(A)|\tau|_{V_{j}}=0 \text { for all } j>n\right\} \text { for all } \beta \in\left(\log \lambda_{n}, \log \lambda_{n+1}\right] .
$$

In particular every $\mathrm{T}_{\beta}(A)$ is weak*-closed. On the other hand the averaging traces $\operatorname{AVT}_{\beta}(A)$ at $\beta=\log \lambda_{G_{s_{0}}}$ correspond to the $\ell^{1}$-normalized eigenvectors of the transpose of

$$
H_{s_{0}}=\left[\begin{array}{cccc}
G_{s_{0}} & * & \cdots & * \\
0 & G_{s_{1}} & \cdots & * \\
\vdots & \vdots & \cdots & \vdots \\
0 & 0 & \cdots & G_{s_{q}}
\end{array}\right]
$$

where $G_{s_{1}}, \ldots, G_{s_{q}}$ are the components communicated by $G_{s_{0}}$. We thus conclude that for every $\beta \in\left(\log \lambda_{n}, \lambda_{n+1}\right]$ with $\log \lambda_{n}, \log \lambda_{n+1} \in \Lambda$ we have an affine weak*-homeomorphism

$$
\Phi:\left\{\tau \in \mathrm{T}(A)|\tau|_{V_{j}}=0 \text { for all } j>n\right\} \rightarrow \mathrm{E}_{\beta}^{\mathrm{fin}}\left(\mathcal{T}_{X}\right),
$$

and for every $\beta=\log \lambda \in \Lambda$ we have an affine weak*-homeomorphism

$$
\Psi:\left\{\tau \in \mathrm{T}(A) \mid H_{s}^{t} \tau=\lambda \tau \text { for some } \lambda \text {-maximal } G_{s}\right\} \rightarrow \mathrm{E}_{\beta}^{\infty}\left(\mathcal{T}_{X}\right) .
$$

From this we can easily read the structure for the graph $\mathrm{C}^{*}$-algebra $\mathcal{O}_{X}$. The map $\Psi$ descends as is since $A \hookrightarrow \mathcal{O}_{X}$ while for $\Phi$ we restrict to the $\tau \in \mathrm{T}_{\beta}(A)$ that have support entirely on sources. We provide a variety of examples for which we compute the above ad-hoc in order to highlight the methods of the proofs. In Section 9 we square our results with [15, Examples 6.1-6.7] by showing how the KMS-simplices can recovered by using entropies.

1.4. States at the upper half plane. We follow [25] and make a distinction between states that are bounded on the upper half plane (ground states) and states that arise at the limit of $\beta \uparrow \infty$ $\left(\mathrm{KMS}_{\infty}\right.$-states). The parametrization in Theorem 10.2 resembles that of $[\mathbf{1 4 , 2 0 , 2 6 , 2 7 ]}$, which in turn are inspired by [23, Theorem 2.2]. Namely, the mapping $\tau \mapsto \varphi_{\tau}$ given by

$$
\varphi_{\tau}(f):= \begin{cases}\tau(f) & \text { if } f \in q_{J}(A) \subseteq \mathcal{O}(J, X), \\ 0 & \text { otherwise, }\end{cases}
$$

defines an affine weak*-homeomorphism from the states $\mathcal{S}(A)$ of $A$ (resp. from $\mathrm{T}(A)$ ) that vanish on $J$, onto the ground states of $\mathcal{O}(J, X)$ (resp. the $\mathrm{KMS}_{\infty}$-states of $\mathcal{O}(J, X)$ ).

\section{Preliminaries}

2.1. Kubo-Martin-Schwinger states. Let $\sigma: \mathbb{R} \rightarrow \operatorname{Aut}(\mathcal{A})$ be an action on a $\mathrm{C}^{*}$-algebra $\mathcal{A}$. Then there exists a norm-dense $\sigma$-invariant $*$-subalgebra $\mathcal{A}_{\text {an }}$ of $\mathcal{A}$ such that for every $f \in \mathcal{A}_{\text {an }}$ the function $\mathbb{R} \ni r \mapsto \sigma_{r}(f) \in \mathcal{A}$ is analytically continued to an entire function $\mathbb{C} \ni z \mapsto \sigma_{z}(f) \in \mathcal{A}$ [3, Proposition 2.5.22]. If $\beta>0$, then a state $\varphi$ of $\mathcal{A}$ is called a $(\sigma, \beta)-K M S$ state (or equilibrium state at $\beta$ ) if it satisfies the KMS-condition:

$$
\varphi(f g)=\varphi\left(g \sigma_{i \beta}(f)\right) \text { for all } f, g \text { in a norm-dense } \sigma \text {-invariant } * \text {-subalgebra of } \mathcal{A}_{\text {an }} \text {. }
$$

If $\beta=0$ or if the action is trivial then a KMS-state is a tracial state on $\mathcal{A}$. The KMS-condition follows as an equivalent for the existence of particular continuous functions [4, Proposition 5.3.7]. More precisely, a state $\varphi$ is an equilibrium state at $\beta>0$ if and only if for any pair $f, g \in \mathcal{A}$ there exists a complex function $F_{f, g}$ that is analytic on $D=\{z \in \mathbb{C} \mid 0<\operatorname{Im}(z)<\beta\}$ and continuous (hence bounded) on $\bar{D}$ such that

$$
F_{f, g}(r)=\varphi\left(f \sigma_{r}(g)\right) \text { and } F_{f, g}(r+i \beta)=\varphi\left(\sigma_{r}(g) f\right) \text { for all } t \in \mathbb{R} .
$$

A state $\varphi$ of $\mathcal{A}$ is called a $K M S_{\infty}$-state if it is the weak*-limit of $(\sigma, \beta)$-KMS states as $\beta \uparrow \infty$. A state $\varphi$ of a $\mathrm{C}^{*}$-algebra $\mathcal{A}$ is called a ground state if the function $z \mapsto \varphi\left(f \sigma_{z}(g)\right)$ is bounded on 
$\{z \in \mathbb{C} \mid \operatorname{Im} z>0\}$ for all $f, g$ inside a dense analytic subset of $\mathcal{A}$. The distinction between ground states and $\mathrm{KMS}_{\infty}$-states is not apparent in [4] and is coined in [25].

2.2. $\mathrm{C}^{*}$-correspondences. The reader should be familiar with the theory of $\mathrm{C}^{*}$-correspondences, e.g. [21]. A $\mathrm{C}^{*}$-correspondence $X$ over $A$ is a right Hilbert $A$-module with a left action given by a *-homomorphism $\phi_{X}: A \rightarrow \mathcal{L} X$. We write $\mathcal{K} X$ for the ideal of compact operators and we denote the rank one compacts by

$$
\theta_{\xi, \eta}: X \rightarrow X: \zeta \mapsto \xi\langle\eta, \zeta\rangle .
$$

For $n>1$ we write $X^{\otimes n}=X^{\otimes n-1} \otimes X$ for the stabilized $n$-tensor product, with the left action given by $\phi_{n}=\phi_{X} \otimes \mathrm{id}_{X \otimes n-1}$. We write $\xi^{\otimes n}:=\xi_{1} \otimes \cdots \otimes \xi_{n}$ for the elementary tensors of $X^{\otimes n}$.

We fix $(\pi, t)$ be the Fock representation of $X$. That is, on $\mathcal{F} X:=\sum^{\oplus^{n}} X^{\otimes n}$ we define the adjointable operators given on the elementary tensors $\eta^{\otimes n} \in X^{\otimes n}$ by

$$
\pi(a) \eta^{\otimes n}=\phi_{n}(a) \eta^{\otimes n} \text { for } a \in A \quad \text { and } t(\xi) \eta^{\otimes n}=\xi \otimes \eta^{\otimes n} \text { for } \xi \in X .
$$

In order to reduce the use of superscripts we will abuse notation and write $t\left(\xi^{\otimes n}\right)$ instead of the more appropriate $t^{n}\left(\xi^{\otimes n}\right)$, and $t\left(\xi^{\otimes 0}\right)=\pi(a)$ for $a=\xi^{\otimes 0} \in A$. We write $\mathcal{T}_{X}$ for the ToeplitzPimsner $\mathrm{C}^{*}$-algebra that is generated by $\pi(A)$ and $t(X)$. It follows that

$$
\mathcal{T}_{X}=\overline{\operatorname{span}}\left\{t\left(\xi^{\otimes n}\right) t\left(\eta^{\otimes m}\right)^{*} \mid \xi^{\otimes n} \in X^{\otimes n}, \eta^{\otimes m} \in X^{\otimes m}, n, m \in \mathbb{Z}_{+}\right\}
$$

with the understanding that $X^{\otimes 0}=A$. It is clear that $\mathcal{T}_{X}$ admits a gauge action $\gamma_{z}:=\operatorname{ad}_{u_{z}}$ given by the unitaries

$$
u_{z}\left(\xi_{n}\right)=z^{n} \xi_{n} \text { for all } \xi_{n} \in X^{\otimes n} .
$$

The Gauge-Invariant-Uniqueness-Theorem (in the full generality obtained by Katsura [21]) asserts that $\mathcal{T}_{X}$ is the universal $\mathrm{C}^{*}$-algebra with respect to pairs $(\rho, v)$ such that

$$
v(\xi)^{*} v(\eta)=\rho(\langle\xi, \eta\rangle) \quad \text { and } \quad \rho(a) t(\xi)=t\left(\phi_{X}(a) \xi\right) .
$$

Any such pair induces a map $\psi_{v}$ on $\mathcal{K} X$ such that $\psi_{v}\left(\theta_{\xi, \eta}\right)=v(\xi) v(\eta)^{*}$. In fact $(\rho, v)$ induces a faithful representation of $\mathcal{T}_{X}$ if and only if it admits a gauge action and $\rho(A) \cap \psi_{v}(\mathcal{K} X)=(0)$ (hence $\rho$ is injective). We also fix the projections

$$
p_{n}: \mathcal{F} X \rightarrow X^{\otimes n} .
$$

It is straightforward that the $p_{n}$ commute with the diagonal operators of $\mathcal{L}(\mathcal{F} X)$ and thus with the elements in the fixed point algebra $\mathcal{T}_{X}^{\gamma}$.

Let $J \subseteq \phi_{X}^{-1}(\mathcal{K} X)$. The $J$-relative Cuntz-Pimsner algebra $\mathcal{O}(J, X)$ is defined as the quotient of $\mathcal{T}_{X}$ by the ideal generated by

$$
\pi(a)-\psi_{t}\left(\phi_{X}(a)\right) \text { for all } a \in J .
$$

As such it inherits the gauge action from $\mathcal{T}_{X}$. In particular $\mathcal{O}(J, X)$ is the universal $\mathrm{C}^{*}$-algebra with respect to pairs $(\rho, v)$ that in addition satisfy the $J$-covariance $\rho(a)=\psi_{v}\left(\phi_{X}(a)\right)$ for all $a \in J$. If $J=J_{X}$ for Katsura's ideal

$$
J_{X}:=\operatorname{ker} \phi_{X}^{\perp} \bigcap \phi_{X}^{-1}(\mathcal{K} X)
$$

then the quotient is the Cuntz-Pimsner algebra $\mathcal{O}_{X}$ [21]. It is shown in [19] that $A$ embeds in $\mathcal{O}(J, X)$ if and only if $J \subseteq J_{X}$. In this case the Gauge-Invariant-Uniqueness-Theorem asserts that a pair $(\rho, v)$ defines a faithful representation of $\mathcal{O}(J, X)$ if and only if it is $J$-covariant, it admits a gauge action, $\rho$ is injective and $J=\left\{a \in A \mid \rho(a) \in \psi_{v}(\mathcal{K} X)\right\}$.

The Fock space itself admits Hilbert spaces quantizations. For convenience we take Hilbert spaces to be conjugate linear in the first entry (so that they are right Hilbert $\mathbb{C}$-modules). Suppose that $\rho_{0}: A \rightarrow \mathcal{B}\left(H_{0}\right)$ is a $*$-representation and form the Hilbert module $\mathcal{F} X \otimes_{\rho_{0}} H_{0}$. It is a Hilbert space with the inner product be given by

$$
\left\langle\xi^{\otimes n} \otimes x, \eta^{\otimes m} \otimes y\right\rangle:=\left\langle x, \rho_{0}\left(\left\langle\xi^{\otimes n}, \eta^{\otimes m}\right\rangle_{\mathcal{F} X}\right) y\right\rangle_{H_{0}}
$$


and the induced pair $(\rho, v):=\left(\pi \otimes I_{H_{0}}, t \otimes I_{H_{0}}\right)$ defines a representation of $\mathcal{T}_{X}$. Now if we consider $\left(H_{\tau}, x_{\tau}, \rho_{\tau}\right)$ be the GNS-representation of $A$ and $\left(H_{u}, \rho_{u}\right)$ be the universal representation of $A$ then $\left(\pi \otimes I_{H_{u}}, t \otimes I_{H_{u}}\right)$ defines a faithful representation of $\mathcal{T}_{X}$ on

$$
\mathcal{F} X \otimes_{\rho_{u}} H_{u} \simeq \sum_{\tau \in \mathcal{S}(A)}^{\oplus} \mathcal{F} X \otimes_{\rho_{\tau}} H_{\tau} .
$$

2.3. The KMS-simplex and the number operator. Fix $s \in \mathbb{R}$. We use the gauge action to define $\sigma: \mathbb{R} \rightarrow \operatorname{Aut}\left(\mathcal{T}_{X}\right)$ by $\sigma_{r}=\gamma_{e^{i r s}}$. It is standard to see then that it extends to an entire function on the analytic elements $f=t\left(\xi^{\otimes n}\right) t\left(\eta^{\otimes m}\right)^{*}$ of $\mathcal{T}_{X}$ by setting

$$
\sigma_{z}\left(t\left(\xi^{\otimes n}\right) t\left(\eta^{\otimes m}\right)^{*}\right)=e^{(n-m) i z s} t\left(\xi^{\otimes n}\right) t\left(\eta^{\otimes m}\right)^{*} .
$$

We emphasize here that we consider just elementary tensors. The $(\sigma, \beta)$-KMS condition for a state $\varphi$ is thus written as

$$
\varphi\left(t\left(\xi^{\otimes n}\right) t\left(\eta^{\otimes m}\right)^{*} \cdot t\left(\zeta^{\otimes k}\right) t\left(y^{\otimes l}\right)^{*}\right)=e^{-(n-m) \beta s} \varphi\left(t\left(\zeta^{\otimes k}\right) t\left(y^{\otimes l}\right)^{*} \cdot t\left(\xi^{\otimes n}\right) t\left(\eta^{\otimes m}\right)^{*}\right)
$$

Likewise we get the $(\sigma, \beta)$-KMS condition for the relative Cuntz-Pimsner algebras $\mathcal{O}(J, X)$.

Definition 2.1. Let $X$ be a $\mathrm{C}^{*}$-correspondence and $J \subseteq \phi_{X}^{-1}(\mathcal{K} X)$. For $\beta>0$ we write $\mathrm{E}_{\beta}(\mathcal{O}(J, X))$ for the set of the $(\sigma, \beta)$-KMS states of $\mathcal{O}(J, X)$ where $\sigma: \mathbb{R} \rightarrow \operatorname{Aut}(\mathcal{O}(J, X))$ is given by $r \mapsto \gamma_{e^{i r}}$ for the gauge action $\gamma$ of $\mathcal{O}(J, X)$.

The rotational action formalizes the distribution $e^{-\beta N}$ for the number operator $N$ given by $N \xi^{\otimes n}=n \xi^{\otimes n}$. This is similar to what is done in Quantum Mechanics and let us include some details here.

Proposition 2.2. Let $X$ be a $C^{*}$-correspondence over $A$ and let $c \in \mathbb{C}$ and $s \in \mathbb{R}$. If $N$ is the number operator on $\mathcal{F} X$ then $e^{i(c+s N)}=e^{i c} u_{e^{i s}}$. Consequently the action $\sigma: \mathbb{R} \rightarrow \operatorname{Aut}\left(\mathcal{T}_{X}\right)$ with

$$
\sigma_{r}(f):=f \mapsto e^{i r(c+s N)} f e^{-i r(c+s N)} \text { for all } f \in \mathcal{T}_{X}
$$

is realized by the rotational action $\mathbb{R} \ni r \mapsto \gamma_{e^{i r s}} \in \operatorname{Aut}\left(\mathcal{T}_{X}\right)$.

Proof. Let $\tau$ be a state of $A$ and form the Hilbert space $\mathcal{F} X \otimes_{\rho_{\tau}} H_{\tau}$ for the GNS-representation $\left(H_{\tau}, x_{\tau}, \rho_{\tau}\right)$ of $\tau$. Then $N \otimes I_{H_{\tau}}=\sum_{k=0}^{\infty} k p_{k} \otimes I_{H_{\tau}}$ is an unbounded selfadjoint operator on $\mathcal{F} X \otimes_{\rho_{\tau}} H_{\tau}$. It suffices to show that

$$
e^{i(c+s N)} \otimes I_{H_{\tau}}=e^{i c} u_{e^{i s}} \otimes I_{H_{\tau}} .
$$

For convenience let us we write $p_{k, \tau}=p_{k} \otimes I_{H_{\tau}}$. By the Spectral Theorem for unbounded normal operators we deduce that

$$
e^{i(c+s N)} \otimes I_{H_{\tau}}=\text { sot- } \lim _{m} e^{i c} \prod_{k=0}^{m} e^{i s k p_{k, \tau}} .
$$

For any $z \in \mathbb{C}$ we can use the functional calculus to approximate $e^{z p_{k, \tau}}$ by $P_{\ell}\left(p_{k, \tau}\right)$ such that the $P_{\ell}(x)=\sum_{j} \alpha_{\ell, j} x^{j}$ converge to $e^{z x}$ for $x \in\{0,1\}$. Then we get

$$
P_{\ell}\left(p_{k, \tau}\right)\left(\xi^{\otimes n} \otimes x_{\tau}\right)=\left\{\begin{array}{ll}
\sum_{j} \alpha_{\ell, j}\left(\xi^{\otimes n} \otimes x_{\tau}\right) & \text { if } n=k, \\
\alpha_{\ell, 0}\left(\xi^{\otimes n} \otimes x_{\tau}\right) & \text { if } n \neq k,
\end{array}= \begin{cases}P_{\ell}(1)\left(\xi^{\otimes n} \otimes x_{\tau}\right) & \text { if } n=k, \\
P_{\ell}(0)\left(\xi^{\otimes n} \otimes x_{\tau}\right) & \text { if } n \neq k .\end{cases}\right.
$$

and so $e^{z p_{k, \tau}}=e^{z} p_{k, \tau}+\sum_{m \neq k} p_{m, \tau}$. Therefore

$$
\begin{aligned}
e^{i(c+s N)} \otimes I_{H_{\tau}}\left(\xi^{\otimes n} \otimes x_{\tau}\right) & =\lim _{m} e^{i c} \prod_{k=0}^{m} e^{i s k p_{k, \tau}\left(\xi^{\otimes n} \otimes x_{\tau}\right)} \\
& =e^{i c} e^{i s n}\left(\xi^{\otimes n} \otimes x_{\tau}\right)=e^{i c}\left(u_{e^{i s}} \otimes I_{H_{\tau}}\right)\left(\xi^{\otimes n} \otimes x_{\tau}\right),
\end{aligned}
$$

and the proof is complete.

Henceforth we focus on the case where $s=1$. Substituting $\beta$ by $s \beta$ in what follows yields the results for any $s \in \mathbb{R}^{+}$. 


\section{Characterization of equilibrium states}

We start by giving an equivalent characterization of the KMS-condition.

Proposition 3.1. Let $X$ be a $C^{*}$-correspondence and let $\beta \in \mathbb{R}$. Then $\varphi \in \mathrm{E}_{\beta}\left(\mathcal{T}_{X}\right)$ if and only if

$$
\varphi\left(t\left(\xi^{\otimes n}\right) t\left(\eta^{\otimes m}\right)^{*}\right)=\delta_{n, m} e^{-n \beta} \varphi\left(t\left(\eta^{\otimes m}\right)^{*} t\left(\xi^{\otimes n}\right)\right)
$$

for all elementary tensor vectors $\xi^{\otimes n} \in X^{\otimes n}, \eta^{\otimes m} \in X^{\otimes m}$, with $n, m \in \mathbb{Z}_{+}$. Consequently two $(\sigma, \beta)-K M S$ states coincide if and only if they agree on $\pi(A)$.

An analogous description holds for the states in $\mathrm{E}_{\beta}(\mathcal{O}(J, X))$ for any relative Cuntz-Pimsner algebra $\mathcal{O}(J, X)$.

Proof. Suppose that $\varphi \in \mathrm{E}_{\beta}\left(\mathcal{T}_{X}\right)$. If $n=m$ then the KMS-condition in (2.2) directly gives that

$$
\varphi\left(t\left(\xi^{\otimes n}\right) t\left(\eta^{\otimes n}\right)^{*}\right)=e^{-n \beta} \varphi\left(t\left(\eta^{\otimes n}\right)^{*} t\left(\xi^{\otimes n}\right)\right) .
$$

If $n \neq m$ then we use that $\varphi$ is $\sigma$-invariant and therefore for every $r \in \mathbb{R}$ we get

$$
\varphi\left(t\left(\xi^{\otimes n}\right) t\left(\eta^{\otimes m}\right)^{*}\right)=\varphi \sigma_{r}\left(t\left(\xi^{\otimes n}\right) t\left(\eta^{\otimes m}\right)^{*}\right)=e^{(n-m) i r} \varphi\left(t\left(\xi^{\otimes n}\right) t\left(\eta^{\otimes m}\right)^{*}\right) .
$$

As $(n-m) \neq 0$ we must have that $\varphi\left(t\left(\xi^{\otimes n}\right) t\left(\eta^{\otimes m}\right)^{*}\right)=0$.

Conversely suppose that $\varphi$ is a state on $\mathcal{T}_{X}$ satisfying (3.1). It will be convenient to refer to elements of the form $t\left(\xi^{\otimes n}\right) t\left(\eta^{\otimes m}\right)^{*}$ as $(n, m)$-products. We have to verify equation (2.2), i.e.,

$$
\varphi\left(t\left(\xi^{\otimes n}\right) t\left(\eta^{\otimes m}\right)^{*} \cdot t\left(\zeta^{\otimes k}\right) t\left(y^{\otimes l}\right)^{*}\right)=e^{-(n-m) \beta} \varphi\left(t\left(\zeta^{\otimes k}\right) t\left(y^{\otimes l}\right)^{*} \cdot t\left(\xi^{\otimes n}\right) t\left(\eta^{\otimes m}\right)^{*}\right) .
$$

We will proceed by considering cases on $n, m, k, l$. The left hand side of $(2.2)$ gives either an $(n, m-k+l)$-product or an $(n+k-m, l)$-product, depending on whether $m \geq k$ or $m \leq k$. Similarly the right hand side gives either a $(k, l-n+m)$-product or a $(k+n-l, m)$-product. In each case we get that $\varphi$ is zero on these products, and thus equation (2.2) holds when $n+k \neq l+m$. Now suppose that $n+k=l+m$. Without loss of generality we may assume that $m \geq k$ and so $n \geq l$ (otherwise take adjoints). Let us write

$$
\eta^{\otimes m}=\eta^{\otimes k} \otimes \eta^{\otimes m-k} \quad \text { and } \quad \xi^{\otimes n}=\xi^{\otimes l} \otimes \xi^{\otimes n-l} .
$$

By using (3.1), the left hand side of (2.2) equals to

$$
\begin{aligned}
\varphi\left(t\left(\xi^{\otimes n}\right) t\left(\eta^{\otimes m}\right)^{*} \cdot t\left(\zeta^{\otimes k}\right) t\left(y^{\otimes l}\right)^{*}\right) & =\varphi\left(t\left(\xi^{\otimes n}\right) t\left(y^{\otimes l} \otimes\left\langle\zeta^{\otimes k}, \eta^{\otimes k}\right\rangle \eta^{\otimes m-k}\right)^{*}\right) \\
& =e^{-n \beta} \varphi\left(t\left(y^{\otimes l} \otimes\left\langle\zeta^{\otimes k}, \eta^{\otimes k}\right\rangle \eta^{\otimes m-k}\right)^{*} t\left(\xi^{\otimes n}\right)\right) \\
& =e^{-n \beta} \varphi\left(t\left(\eta^{\otimes m}\right)^{*} t\left(\zeta^{\otimes k}\right) t\left(y^{\otimes l}\right)^{*} t\left(\xi^{\otimes n}\right)\right) .
\end{aligned}
$$

Likewise, the right hand side of equation 2.2 equals to

$$
\begin{aligned}
e^{-(n-m) \beta} \varphi\left(t\left(\zeta^{\otimes k}\right) t\left(y^{\otimes l}\right)^{*} \cdot t\left(\xi^{\otimes n}\right) t\left(\eta^{\otimes m}\right)^{*}\right) & =e^{-(n-m) \beta} \varphi\left(t\left(\zeta^{\otimes k} \otimes\left\langle y^{\otimes l}, \xi^{\otimes l}\right\rangle \xi^{\otimes n-l}\right) t\left(\eta^{\otimes m}\right)^{*}\right) \\
& =e^{-(n-m) \beta} e^{-m \beta} \varphi\left(t\left(\eta^{\otimes m}\right)^{*} t\left(\zeta^{\otimes k} \otimes\left\langle y^{\otimes l}, \xi^{\otimes l}\right\rangle \xi^{\otimes n-l}\right)\right) \\
& =e^{-n \beta} \varphi\left(t\left(\eta^{\otimes m}\right)^{*} t\left(\zeta^{\otimes k}\right) t\left(y^{\otimes l}\right)^{*} t\left(\xi^{\otimes n}\right)\right) .
\end{aligned}
$$

Therefore equation 2.2 is satisfied, and the proof is complete.

The following proposition allows us to consider just unital $\mathrm{C}^{*}$-correspondences from now on. When $\phi_{X}$ is not unital, we define $X^{1}$ be the space $X$ which becomes a $\mathrm{C}^{*}$-correspondence over $A^{1}=A+\mathbb{C}$ by extending the operations $\phi_{X}(1) \xi=\xi=\xi 1$. Note here that $A^{1}=A \oplus \mathbb{C}$ when $A$ is already unital but $\phi_{X}\left(1_{A}\right) \neq 1_{X}$.

Proposition 3.2. Let $X$ be a $C^{*}$-correspondence over $A$. Then $\varphi$ is a $(\sigma, \beta)$-KMS state for $\mathcal{T}_{X^{1}}$ if and only if it restricts to a $(\sigma, \beta)-K M S$ state on $\mathcal{T}_{X}$.

Proof. If $\phi_{X}: A \rightarrow \mathcal{L} X$ is unital then there is nothing to show. Otherwise let $(\pi, t)$ be the Fock representation of $X^{1}$ and notice that $\left(\left.\pi\right|_{A}, t\right)$ defines a faithful representation of $\mathcal{T}_{X}$ by the Gauge-Invariant-Uniqueness-Theorem. Indeed it admits a gauge action and if $\pi(a) \in \psi_{t}(\mathcal{K} X)$ then

$$
a=p_{0} \pi(a) p_{0} \in p_{0} \psi_{t}(\mathcal{K} X) p_{0} \subseteq p_{0} \psi_{t}\left(\mathcal{K} X^{1}\right) p_{0}=(0)
$$


for the projection $p_{0}$ on $A^{1} \subset \mathcal{F} X^{1}$. Therefore $\mathcal{T}_{X} \subseteq \mathcal{T}_{X^{1}}$. In fact we see that $\mathcal{T}_{X^{1}}$ is the unitization of $\mathcal{T}_{X}$. As the $(\sigma, \beta)$-KMS condition is the same for both $\mathcal{T}_{X}$ and $\mathcal{T}_{X^{1}}$ then the equivalence follows by the unitization of states. Notice here that $\sigma$ is the same action spatially implemented by the corresponding unitaries.

Remark 3.3. Henceforth we will assume that the $\mathrm{C}^{*}$-correspondence is unital for our proofs. However the statements will be given for possibly non-unital $\mathrm{C}^{*}$-correspondences.

\section{WOLD DECOMPOSITION}

We will consider $\mathrm{C}^{*}$-correspondences that admit a finite decomposition of unit. By Kasparov's Stabilization Theorem this is equivalent to having $\mathcal{L} X=\mathcal{K} X$.

Definition 4.1. A $\mathrm{C}^{*}$-correspondence $X$ over $A$ will be of finite rank if there is a finite collection $x:=\left\{x_{1}, \ldots, x_{d}\right\}$ of vectors in the unit ball of $X$ such that $\sum_{i \in[d]} \theta_{x_{i}, x_{i}}=1_{X}$.

Remark 4.2. For any non-trivial word $\mu=\mu_{n} \cdots \mu_{1} \in \mathbb{F}_{+}^{d}$ we write

$$
x_{\mu}:=x_{\mu_{n}} \otimes \cdots \otimes x_{\mu_{1}} \in X^{\otimes n} .
$$

We reserve the notation $x_{\emptyset}=1_{A} \in X^{\otimes 0}$ when $A$ is unital. It follows that $X^{\otimes n}$ has finite rank with respect to the collection $\left\{x_{\mu}\left|\mu \in \mathbb{F}_{+}^{d},\right| \mu \mid=n\right\}$.

Remark 4.3. By construction, $\mathcal{K}(\mathcal{F} X)$ is an ideal in $\mathcal{T}_{X}$. When $X$ is of finite rank then we can write every projection $p_{k}: \mathcal{F} X \rightarrow A$ with $k \geq 1$ by

$$
p_{k}=\sum_{|\mu|=k} t\left(x_{\mu}\right) t\left(x_{\mu}\right)^{*}-\sum_{|\nu|=k+1} t\left(x_{\nu}\right) t\left(x_{\nu}\right)^{*},
$$

and thus $p_{k} \in \mathcal{T}_{X}$ for all $k \geq 1$. Moreover we see that

$$
p_{0}=1_{\mathcal{F} X}-\sum_{i \in[d]} t\left(x_{i}\right) t\left(x_{i}\right)^{*} \in \mathcal{T}_{X^{1}} .
$$

Hence by using the unitization we have that $p_{k} \in \mathcal{T}_{X}^{1}$ for all $k \in \mathbb{Z}_{+}$. It is straightforward that the $p_{n}$ commute with all elements in $\mathcal{T}_{X^{1}}^{\gamma}$, as the latter are supported on the diagonal of $\mathcal{F} X$. Moreover if $\varphi \in \mathrm{E}_{\beta}\left(\mathcal{T}_{X^{1}}\right)$ then

$$
\varphi\left(p_{k}\right)=\sum_{|\mu|=k} \varphi\left(t\left(x_{\mu}\right) p_{0} t\left(x_{\mu}\right)^{*}\right)=\sum_{|\mu|=k} e^{-k \beta} \varphi\left(p_{0} \pi\left(\left\langle x_{\mu}, x_{\mu}\right\rangle\right) p_{0}\right) \leq \sum_{|\mu|=k} e^{-k \beta} \varphi\left(p_{0}\right) .
$$

Therefore if $\varphi\left(p_{0}\right)=0$ then $\varphi\left(p_{k}\right)=0$ for all $k \in \mathbb{Z}_{+}$.

This triggers the following definition. We will be using the same symbol for the extension of a state from $\mathcal{T}_{X}$ to $\mathcal{T}_{X^{1}}$ from Proposition 3.2.

Definition 4.4. Let $X$ be a $C^{*}$-correspondence of finite rank over $A$. For $\beta>0$ we define

$$
\mathrm{E}_{\beta}^{\mathrm{fin}}\left(\mathcal{T}_{X}\right):=\left\{\varphi \in \mathrm{E}_{\beta}\left(\mathcal{T}_{X}\right) \mid \sum_{k=0}^{\infty} \varphi\left(p_{k}\right)=1\right\} \text { and } \mathrm{E}_{\beta}^{\infty}\left(\mathcal{T}_{X}\right):=\left\{\varphi \in \mathrm{E}_{\beta}\left(\mathcal{T}_{X}\right) \mid \varphi\left(p_{0}\right)=0\right\}
$$

Likewise we define $\mathrm{E}_{\beta}^{\infty}(\mathcal{O}(J, X))$ and $\mathrm{E}_{\beta}^{\mathrm{fin}}(\mathcal{O}(J, X))$ for any $J$-relative Cuntz-Pimsner algebra with respect to the projections $q_{J}\left(p_{k}\right)$, for the canonical *-epimorphism $q_{J}: \mathcal{T}_{X} \rightarrow \mathcal{O}(J, X)$.

Remark 4.5. We note that $\mathrm{E}_{\beta}^{\infty}(\cdot)$ and $\mathrm{E}_{\beta}^{\text {fin }}(\cdot)$ may be trivial in some cases. For if $q: \mathcal{T}_{X} \rightarrow \mathcal{O}(A, X)$ is the canonical $*$-epimorphism, then its kernel $\mathcal{K}(\mathcal{F} X)$ is generated by $p_{0}$. This automatically implies that $\mathrm{E}_{\beta}^{\mathrm{fin}}(\mathcal{O}(A, X))=\emptyset$. As another example, in Proposition 5.7 we will show that $\mathrm{E}_{\beta}^{\infty}\left(\mathcal{T}_{X}\right)=\emptyset$ for sufficiently large $\beta$.

Notice that $\mathcal{K}(\mathcal{F} X) \subseteq \mathcal{T}_{X}$ and thus it inherits the gauge action by restriction. Therefore we also get equilibrium states for $\mathcal{K}(\mathcal{F} X)$. Let us give an alternative proof of [23, Proposition 2.4] of the Wold decomposition into a finite and an infinite part. 
Theorem 4.6. Let $X$ be a $C^{*}$-correspondence of finite rank over $A$ and let $\beta>0$. Then for any $\varphi \in \mathrm{E}_{\beta}\left(\mathcal{T}_{X}\right)$ we have:

(i) $\varphi \in \mathrm{E}_{\beta}^{\text {fin }}\left(\mathcal{T}_{X}\right)$ if and only if $\left.\varphi\right|_{\mathcal{K}(\mathcal{F} X)} \in \mathrm{E}_{\beta}(\mathcal{K}(\mathcal{F} X))$;

(ii) $\varphi \in \mathrm{E}_{\beta}^{\infty}\left(\mathcal{T}_{X}\right)$ if and only if $\left.\varphi\right|_{\mathcal{K}(\mathcal{F} X)}=0$ if and only if $\varphi$ factors through $\mathcal{O}(A, X)$;

(iii) There are unique $\varphi_{\text {fin }} \in \mathrm{E}_{\beta}^{\text {fin }}\left(\mathcal{T}_{X}\right)$ and $\varphi_{\infty} \in \mathrm{E}_{\beta}^{\infty}\left(\mathcal{T}_{X}\right)$ such that

$$
\varphi=\lambda \varphi_{\mathrm{fin}}+(1-\lambda) \varphi_{\infty}, \text { for } \lambda:=\sum_{k=0}^{\infty} \varphi\left(p_{k}\right) .
$$

Proof. By positivity we have $\sum_{k=0}^{n} \varphi\left(p_{k}\right) \leq 1$ for every $n \in \mathbb{N}$ and so $\sum_{k=0}^{\infty} \varphi\left(p_{k}\right)<\infty$. Moreover equation (4.2) implies that $\sum_{k=0}^{\infty} \varphi\left(p_{k}\right)=0$ if and only if $p_{0}=0$. Now both items (i) and (ii) follow by using $\left(\sum_{k=0}^{n} p_{k}\right)_{n}$ as a contractive approximate identity of $\mathcal{K}(\mathcal{F} X)$. For item (iii) use the Wold decomposition with respect to the quotient map $\mathcal{T}_{X} \rightarrow \mathcal{O}(A, X)$ and the KMS-condition on $\varphi$ to define the positive functional $\psi_{\text {fin }}: \mathcal{T}_{X} \rightarrow \mathbb{C}$ by

$$
\psi_{\text {fin }}(f):=\sum_{k, \ell=0}^{\infty} \varphi\left(p_{k} f p_{\ell}\right)=\sum_{k, \ell=0}^{\infty} \varphi\left(p_{k} f p_{\ell} p_{k}\right)=\sum_{k=0}^{\infty} \varphi\left(p_{k} f p_{k}\right),
$$

and let

$$
\psi_{\infty}(f):=\varphi(f)-\psi_{\text {fin }}(f)
$$

for $f \in \mathcal{T}_{X}$. If $\psi_{\text {fin }} \neq 0$ then $\lambda:=\sum_{k=0}^{\infty} \varphi\left(p_{k}\right)=\left\|\psi_{\text {fin }}\right\|$, and so $\left\|\psi_{\infty}\right\|=1-\lambda$. Hence if $\lambda \in(0,1)$ we obtain the states

$$
\varphi_{\text {fin }}:=\lambda^{-1} \psi_{\text {fin }} \quad \text { and } \quad \varphi_{\infty}:=(1-\lambda)^{-1} \psi_{\infty} .
$$

Since there is a unique extension of a state from $\mathcal{K}(\mathcal{F} X)$ to $\mathcal{T}_{X}$ we get uniqueness of this decomposition. As $\varphi_{\infty}\left(p_{0}\right)=0$ it remains to show that $\varphi_{\text {fin }}$ and $\varphi_{\infty}$ satisfy the KMS-condition. Equivalently that $\psi_{\text {fin }}$ does so. By definition we have that $\psi_{\text {fin }}\left(t\left(\xi^{\otimes n}\right) t\left(\eta^{\otimes m}\right)^{*}\right)=0$ when $n \neq m$. Now if $n=m$ then we get

$$
t\left(\eta^{\otimes n}\right)^{*} p_{k} t\left(\xi^{\otimes n}\right)= \begin{cases}p_{k-n} t\left(\eta^{\otimes n}\right)^{*} t\left(\xi^{\otimes n}\right) p_{k-n} & \text { if } k \geq n, \\ 0 & \text { otherwise. }\end{cases}
$$

Therefore for all $n, m \in \mathbb{Z}_{+}$we obtain

$$
\begin{aligned}
\psi_{\text {fin }}\left(t\left(\xi^{\otimes n}\right) t\left(\eta^{\otimes m}\right)^{*}\right) & =\delta_{n, m} \sum_{k=0}^{\infty} \varphi\left(p_{k} t\left(\xi^{\otimes n}\right) t\left(\eta^{\otimes m}\right)^{*} p_{k}\right) \\
& =\delta_{n, m} e^{-n \beta} \sum_{k=0}^{\infty} \varphi\left(t\left(\eta^{\otimes m}\right)^{*} p_{k} t\left(\xi^{\otimes n}\right)\right) \\
& =\delta_{n, m} e^{-n \beta} \sum_{k \geq n}^{\infty} \varphi\left(p_{k-n} t\left(\eta^{\otimes m}\right)^{*} t\left(\xi^{\otimes n}\right) p_{k-n}\right) \\
& =\delta_{n, m} e^{-n \beta} \sum_{k=0}^{\infty} \varphi\left(p_{k} t\left(\eta^{\otimes m}\right)^{*} t\left(\xi^{\otimes n}\right) p_{k}\right)=\delta_{n, m} \psi_{\text {fin }}\left(t\left(\eta^{\otimes m}\right)^{*} t\left(\xi^{\otimes n}\right)\right)
\end{aligned}
$$

and thus $\psi_{\text {fin }}$ satisfies equation (3.1).

Remark 4.7. The convex decomposition is not weak*-continuous. For example, for fixed $\varphi_{\infty} \in$ $\mathrm{E}_{\beta}^{\infty}\left(\mathcal{T}_{X}\right)$ and $\varphi_{\mathrm{fin}} \in \mathrm{E}_{\beta}^{\mathrm{fin}}\left(\mathcal{T}_{X}\right)$, the states $\varphi_{n}=n^{-1} \varphi_{\mathrm{fin}}+\left(1-n^{-1}\right) \varphi_{\infty}$ weak*-converge to $\varphi_{\infty}$. However the infinite and the finite parts of all $\varphi_{n}$ stay the same.

\section{ENTROPY}

We start with a remark that ensures that the quantities we are to introduce are independent of the choice of the unit decomposition. 
Remark 5.1. Let $\left\{x_{1}, \ldots, x_{d}\right\}$ and $\left\{y_{1}, \ldots, y_{d^{\prime}}\right\}$ be two unit decompositions. Then for $\tau \in \mathrm{T}(A)$ we get that

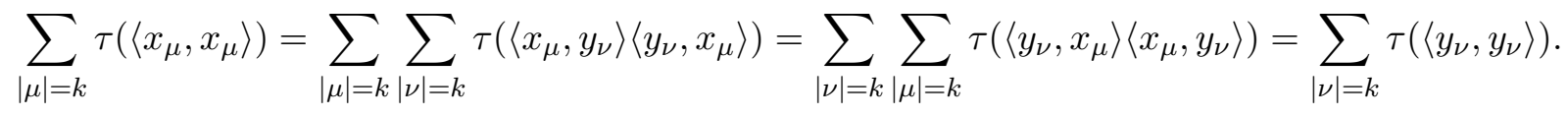

That is, the value $\sum_{|\mu|=k} \tau\left(\left\langle x_{\mu}, x_{\mu}\right\rangle\right)$ is independent of the unit decomposition.

Definition 5.2. Let $X$ be a $C^{*}$-correspondence of finite rank over $A$ with respect to $\left\{x_{1}, \ldots, x_{d}\right\}$ and let $\beta>0$. For any $\tau \in \mathrm{T}(A)$ we define the formal series

$$
c_{\tau, \beta}:=\sum_{k=0}^{\infty} e^{-k \beta} \sum_{|\mu|=k} \tau\left(\left\langle x_{\mu}, x_{\mu}\right\rangle\right) .
$$

We write $\mathrm{T}_{\beta}(A):=\left\{\tau \in \mathrm{T}(A) \mid c_{\tau, \beta}<\infty\right\}$.

Remark 5.1 implies that $c_{\tau, \beta}$ and $\mathrm{T}_{\beta}(A)$ do not depend on the unit decomposition. Since $x_{\emptyset}=1_{A}$ then we see that $c_{\tau, \beta} \geq 1$. Moreover the set $\mathrm{T}_{\beta}(A)$ is convex. On the other extreme we have the notion of averages.

Definition 5.3. Let $X$ be a $C^{*}$-correspondence of finite rank over $A$ with respect to $\left\{x_{1}, \ldots, x_{d}\right\}$ and let $\beta>0$. Let $\operatorname{AVT}_{\beta}(A)$ be the set of the tracial states $\tau$ of $A$ that satisfy

$$
\tau(a)=e^{-\beta} \sum_{i \in[d]} \tau\left(\left\langle x_{i}, a x_{i}\right\rangle\right) \text { for all } a \in A .
$$

As in Remark 5.1 we have that $\operatorname{AVT}_{\beta}(A)$ does not depend on the decomposition $\left\{x_{1}, \ldots, x_{d}\right\}$ of the unit. The next proposition marks that $\mathrm{T}_{\beta}(A) \cap \operatorname{AVT}_{\beta}(A)=\emptyset$.

Proposition 5.4. Let $X$ be a $C^{*}$-correspondence of finite rank over $A$ and let $\beta>0$. If $\tau \in$ $\operatorname{AVT}_{\beta}(A)$ then $c_{\tau, \beta}=\infty$.

Proof. Induction yields an average formula for all words of length $k$, i.e.,

$$
\tau(a)=e^{-k \beta} \sum_{|\mu|=k} \tau\left(\left\langle x_{\mu}, a x_{\mu}\right\rangle\right) \text { for all } a \in A, k \in \mathbb{N} .
$$

The root test implies a notion of entropy for $\tau \in \mathrm{T}(A)$ that connects with convergence of $c_{\tau, \beta}$. We are going to use also two notions of entropy for $X$. As we use entropy for convergence of $c_{\tau, \beta}$ we set $\lim \sup _{k} k^{-1} \log a_{k}=0$ if $a_{k}=0$ eventually.

Definition 5.5. Let $X$ be a $C^{*}$-correspondence of finite rank over $A$ with respect to a unit decomposition $x=\left\{x_{1}, \ldots, x_{d}\right\}$.

(1) The entropy of a $\tau \in \mathrm{T}(A)$ is given by

$$
h_{X}^{\tau}:=\limsup _{k} \frac{1}{k} \log \sum_{|\mu|=k} \tau\left(\left\langle x_{\mu}, x_{\mu}\right\rangle\right) .
$$

(2) The entropy of $x=\left\{x_{1}, \ldots, x_{d}\right\}$ is defined by

$$
h_{X}^{x}:=\limsup _{k} \frac{1}{k} \log \left\|\sum_{|\mu|=k}\left\langle x_{\mu}, x_{\mu}\right\rangle\right\|_{A} .
$$

(3) The strong entropy of $X$ is defined by

$$
h_{X}^{s}:=\inf \left\{h_{X}^{x} \mid x=\left\{x_{1}, \ldots, x_{d}\right\} \text { is a unit decomposition for } X\right\} .
$$

(4) The entropy of $X$ is defined by

$$
h_{X}:=\inf \left\{\beta>0 \mid \mathrm{E}_{\beta}\left(\mathcal{T}_{X}\right) \neq \emptyset\right\} .
$$


Remark 5.6. Due to remark 5.1, the entropy $h_{X}^{\tau}$ is independent of the unit decomposition. Likewise $h_{X}^{s}=h_{X}^{x}$ for any unit decomposition when $A$ is abelian. Furthermore the limsup in $h_{X}^{x}$ is actually the limit of a decreasing sequence. Indeed for $k_{1}, k_{2} \in \mathbb{N}$ with $k_{1}+k_{2}=k$ we get

$$
\begin{aligned}
\sum_{\mu=k}\left\langle x_{\mu}, x_{\mu}\right\rangle & =\sum_{\left|\nu_{1}\right|=k_{1},\left|\nu_{2}\right|=k_{2}}\left\langle x_{\nu_{1}} \otimes x_{\nu_{2}}, x_{\nu_{1}} \otimes x_{\nu_{2}}\right\rangle \\
& =\sum_{\left|\nu_{1}\right|=k_{1}}\left\langle x_{\nu_{1}},\left(\sum_{\left|\nu_{2}\right|=k_{2}}\left\langle x_{\nu_{2}}, x_{\nu_{2}}\right\rangle\right)\left(x_{\nu_{1}}\right)\right\rangle \leq\left\|\sum_{\left|\nu_{2}\right|=k_{2}}\left\langle x_{\nu_{2}}, x_{\nu_{2}}\right\rangle\right\|_{A} \sum_{\left|\nu_{1}\right|=k_{1}}\left\langle x_{\nu_{1}}, x_{\nu_{1}}\right\rangle .
\end{aligned}
$$

Therefore the sequence $\left\|\sum_{|\mu|=k}\left\langle x_{\mu}, x_{\mu}\right\rangle\right\|_{A}$ is submultiplicative.

We close this section with a connection between entropies and $\mathrm{E}_{\beta}\left(\mathcal{T}_{X}\right)$. We shall see later that Proposition 5.7(iv) can follow from the complete parametrization of $\mathrm{E}_{\beta}^{\mathrm{fin}}\left(\mathcal{T}_{X}\right)$ and $\mathrm{E}_{\beta}^{\infty}\left(\mathcal{T}_{X}\right)$. Item (v) below is basically a rewording of [34, Theorem 2.5 and Corollary 2.6].

Proposition 5.7. Let $X$ be a $C^{*}$-correspondence of finite rank over $A$ and let $\beta>0$.

(i) If $\tau \in \mathrm{T}_{\beta}(A) \cup \operatorname{AVT}_{\beta}(A)$ then $h_{X}^{\tau} \leq \beta$.

(ii) For every $\tau \in \mathrm{T}(A)$ we have that $h_{X}^{\tau} \leq h_{X}^{s} \leq \log d$.

(iii) $\mathrm{T}_{\beta}(A)=\mathrm{T}(A)$ whenever $\beta>h_{X}^{s}$.

(iv) $\mathrm{E}_{\beta}^{\infty}\left(\mathcal{T}_{X}\right)=\emptyset$ and $\mathrm{E}_{\beta}\left(\mathcal{T}_{X}\right)=\mathrm{E}_{\beta}^{\mathrm{fin}}\left(\mathcal{T}_{X}\right)$ whenever $\beta>h_{X}^{s}$.

(v) If $A$ is abelian and $h_{X}^{s}>0$ then $\operatorname{AVT}_{h_{X}^{s}}(A) \neq \emptyset$.

Proof. Let $x=\left\{x_{1}, \ldots, x_{d}\right\}$ be a decomposition of the unit. Item (i) follows directly from the root test when $\tau \in \mathrm{T}_{\beta}(A)$ and from Proposition 5.4 when $\tau \in \operatorname{AVT}_{\beta}(A)$. Moreover it is straightforward to check that if $\tau \in \mathrm{T}(A)$ then

$$
\sum_{|\mu|=k} \tau\left(\left\langle x_{\mu}, x_{\mu}\right\rangle\right) \leq\left\|\sum_{|\mu|=k}\left\langle x_{\mu}, x_{\mu}\right\rangle\right\|_{A} \leq d^{k} .
$$

As the left hand side does not depend on $x$, taking infimum over all unit decompositions gives that $h_{X}^{\tau} \leq h_{X}^{s} \leq \log d$. For item (iii) suppose that $\beta \in\left(h_{X}^{s}, \infty\right)$ and choose a unit decomposition $x=\left\{x_{1}, \ldots, x_{d}\right\}$ such that $h_{X}^{s} \leq h_{X}^{x}<\beta$. Then for any $\tau \in \mathrm{T}(A)$ we have that

$$
\underset{k}{\limsup }\left(e^{-k \beta} \sum_{|\mu|=k} \tau\left(\left\langle x_{\mu}, x_{\mu}\right\rangle\right)^{1 / k} \leq e^{-\beta} e^{h_{X}^{x}}<1\right.
$$

giving that $c_{\tau, \beta}<\infty$. For item (iv), if $\varphi \in \mathrm{E}_{\beta}^{\infty}\left(\mathcal{T}_{X}\right)$ then $\varphi\left(p_{0}\right)=0$ and thus $\varphi\left(p_{k}\right)=0$ for all $k \in \mathbb{Z}_{+}$by equation (4.2). But then the KMS-condition yields

$$
1=\sum_{|\mu|=k} \varphi\left(t\left(x_{\mu}\right) t\left(x_{\mu}\right)^{*}\right)=e^{-k \beta} \sum_{|\mu|=k} \varphi \pi\left(\left\langle x_{\mu}, x_{\mu}\right\rangle\right) \leq e^{-k \beta}\left\|\sum_{|\mu|=k}\left\langle x_{\mu}, x_{\mu}\right\rangle\right\|_{A},
$$

as $\varphi \pi \in \mathrm{T}(A)$. Hence $\beta \leq k^{-1} \log \left\|\sum_{|\mu|=k}\left\langle x_{\mu}, x_{\mu}\right\rangle\right\|_{A}$ for all $k \in \mathbb{Z}_{+}$, which gives that $\beta \leq h_{X}^{x}$. Taking the infimum over all unit decompositions yields $\beta \leq h_{X}^{s}$. Finally, if $A$ is abelian then the arguments of [34, Theorem 2.5 and Corollary 2.6] apply to give that $\operatorname{AVT}_{h_{X}^{s}}(A) \neq \emptyset$. In short let the map

$$
\psi: A \rightarrow A \text { such that } \psi(a)=\sum_{i \in[d]}\left\langle x_{i}, a x_{i}\right\rangle .
$$

As $\psi$ is a positive map we have $\left\|\psi^{k}\right\|=\left\|\psi^{k}(1)\right\|=\left\|\sum_{|\mu|=k}\left\langle x_{\mu}, x_{\mu}\right\rangle\right\|_{A}$ for all $k \in \mathbb{N}$. Therefore

$$
h_{X}^{s}=\lim _{k} \log \left\|\psi^{k}\right\|^{1 / k}=\log \lambda_{\psi},
$$

where $\lambda_{\psi}$ is the spectral radius of $\psi$. Then [34, Theorem 2.5 and Corollary 2.6] imply that $\lambda_{\psi}$ is an eigenvalue of the adjoint of $\psi$ on the states of $A$, i.e., there is $\tau \in \mathcal{S}(A)$ such that $\tau \psi=\lambda_{\psi} \tau \psi$ (the fullness condition of [34] is not required here). Hence $\tau$ gives a tracial state in $\operatorname{AVT}_{h_{X}^{s}}(A)$. 


\section{The Finite PART OF THE EQUILIBRiUm STATES}

In this section we parametrize the states in $\mathrm{E}_{\beta}^{\mathrm{fin}}\left(\mathcal{T}_{X}\right)$ for $\beta>0$ and consequently we show how this induces a parametrization for all $\mathrm{E}_{\beta}^{\text {fin }}(\mathcal{O}(J, X))$. Passing from $\mathrm{T}_{\beta}(A)$ to $\mathrm{E}_{\beta}^{\text {fin }}\left(\mathcal{T}_{X}\right)$ uses essentially [23, proof of Theorem 2.1]. Showing that this construction is a bijection generalizes the corresponding arguments from [25].

Theorem 6.1. Let $X$ be a $C^{*}$-correspondence of finite rank over $A$ and let $\beta>0$. Then there is a bijection

$$
\Phi: \mathrm{T}_{\beta}(A) \rightarrow \mathrm{E}_{\beta}^{\mathrm{fin}}\left(\mathcal{T}_{X}\right) \text { such that } \Phi(\tau)\left(p_{0}\right)=c_{\tau, \beta}^{-1} .
$$

If $x=\left\{x_{1}, \ldots, x_{d}\right\}$ is a decomposition of the unit then $\Phi$ is given by

$$
\Phi(\tau)\left(t\left(\xi^{\otimes n}\right) t\left(\xi^{\otimes m}\right)^{*}\right)=\delta_{n, m} c_{\tau, \beta}^{-1} \sum_{\mu \in \mathbb{F}_{+}^{d}} e^{-(|\mu|+n) \beta} \tau\left(\left\langle\eta^{\otimes m} \otimes x_{\mu}, \xi^{\otimes n} \otimes x_{\mu}\right\rangle\right)
$$

for all $\xi^{\otimes n} \in X^{\otimes n}$ and $\eta^{\otimes m} \in X^{\otimes m}$. If, in addition, $\mathrm{E}_{\beta}^{\mathrm{fin}}\left(\mathcal{T}_{X}\right)$ is weak*-closed then $\Phi$ is a weak ${ }^{*}$ homeomorphism between weak*-compact sets.

Proof. Equation (6.1) is independent of the unit decomposition for $\tau \in \mathrm{T}_{\beta}(A)$. Indeed let $y=$ $\left\{y_{1}, \ldots, y_{d^{\prime}}\right\}$ be a second decomposition. If $n \neq m$ then there is nothing to show. For $n=m$ we directly verify that

$$
\begin{aligned}
\sum_{|\mu|=k} \tau\left(\left\langle\eta^{\otimes n} \otimes x_{\mu}, \xi^{\otimes n} \otimes x_{\mu}\right\rangle\right) & =\sum_{|\mu|=k} \sum_{|\nu|=k} \tau\left(\left\langle\eta^{\otimes n} \otimes x_{\mu}, \xi^{\otimes n} \otimes \theta_{y_{\nu}, y_{\nu}} x_{\mu}\right\rangle\right. \\
& =\sum_{|\mu|=k} \sum_{|\nu|=k} \tau\left(\left\langle x_{\mu},\left\langle\eta^{\otimes n}, \xi^{\otimes n}\right\rangle y_{\nu}\right\rangle\left\langle y_{\nu}, x_{\mu}\right\rangle\right) \\
& =\sum_{|\nu|=k} \sum_{|\mu|=k} \tau\left(\left\langle y_{\nu}, x_{\mu}\right\rangle\left\langle x_{\mu},\left\langle\eta^{\otimes n}, \xi^{\otimes n}\right\rangle y_{\nu}\right\rangle\right) \\
& =\sum_{|\nu|=k} \tau\left(\left\langle\eta^{\otimes n} \otimes y_{\nu}, \xi^{\otimes n} \otimes y_{\nu}\right\rangle\right) .
\end{aligned}
$$

Now we proceed to the construction of $\Phi$. First we show that $\varphi_{\tau} \equiv \Phi(\tau)$ exists and is in $\mathrm{E}_{\beta}^{\mathrm{fin}}\left(\mathcal{T}_{X}\right)$ when $\tau \in \mathrm{T}_{\beta}(A)$. Let $\left(H_{\tau}, x_{\tau}, \rho_{\tau}\right)$ be the GNS-representation associated to $\tau$ and consider the induced pair $(\rho, v):=(\pi \otimes I, t \otimes I)$ for $\mathcal{T}_{X}$ acting on $\mathcal{F} X \otimes_{\rho_{\tau}} H_{\tau}$. For any word $\mu$ on the $d$ symbols define the positive vector state $\varphi_{\tau, \mu}$ of $\mathcal{T}_{X}$ be given by

$$
\varphi_{\tau, \mu}(f)=\left\langle x_{\mu} \otimes x_{\tau},(\rho \times v)(f) x_{\mu} \otimes x_{\tau}\right\rangle_{H} \text { for } f \in \mathcal{T}_{X} .
$$

We then define

$$
\varphi_{\tau}:=c_{\tau, \beta}^{-1} \sum_{k=0}^{\infty} e^{-k \beta} \sum_{|\mu|=k} \varphi_{\tau, \mu} .
$$

To see that it is indeed well defined (and a state) on $\mathcal{T}_{X}$ first check that

$$
c_{\tau, \beta}^{-1} \sum_{k=0}^{\infty} e^{-k \beta} \sum_{|\mu|=k} \varphi_{\tau, \mu}\left(\pi\left(1_{A}\right)\right)=c_{\tau, \beta}^{-1} \sum_{k=0}^{\infty} e^{-k \beta} \sum_{|\mu|=k} \tau\left(\left\langle x_{\mu}, x_{\mu}\right\rangle\right)=1 .
$$

Likewise we have $\varphi_{\tau, \mu}(f) \leq\|f\| \varphi_{\tau, \mu}\left(\pi\left(1_{A}\right)\right)$ for all $0 \leq f \in \mathcal{T}_{X}$, and thus

$$
c_{\tau, \beta}^{-1} \sum_{k=0}^{\infty} e^{-k \beta} \sum_{|\mu|=k} \varphi_{\tau, \mu}(f) \leq c_{\tau, \beta}^{-1} \sum_{k=0}^{\infty} e^{-k \beta} \sum_{|\mu|=k}\|f\| \cdot \varphi_{\tau, \mu}\left(\pi\left(1_{A}\right)\right)=\|f\| .
$$

Next we show that $\varphi_{\tau}$ satisfies equation (6.1). If $n \neq m$ then for all $\mu$ we get that

$$
\varphi_{\tau, \mu}\left(t\left(\xi^{\otimes n}\right) t\left(\eta^{\otimes m}\right)^{*}\right)=\tau\left(\left\langle t\left(\xi^{\otimes n}\right)^{*} x_{\mu}, t\left(\eta^{\otimes m}\right)^{*} x_{\mu}\right\rangle_{\mathcal{F} X}\right)=0,
$$

and thus $\varphi_{\tau}\left(t\left(\xi^{\otimes n}\right) t\left(\eta^{\otimes m}\right)^{*}\right)=0$. If $n=m$ and $k \geq n$, then for all $x_{\mu}$ with $|\mu|<n$ we get that

$$
\varphi_{\tau, \mu}\left(t\left(\xi^{\otimes n}\right) t\left(\eta^{\otimes n}\right)^{*}\right)=\tau\left(\left\langle t\left(\xi^{\otimes n}\right)^{*} x_{\mu}, t\left(\eta^{\otimes n}\right)^{*} x_{\mu}\right\rangle_{\mathcal{F} X}\right)=0 .
$$


On the other hand if $|\mu|=k \geq n$ then recall that $\sum_{|\mu|=k} t\left(x_{\mu}\right) t\left(x_{\mu}\right)^{*}$ acts as a unit on $t\left(X^{\otimes \ell}\right)$ for all $\ell \geq k$. Thus we get

$$
\begin{aligned}
\sum_{|\mu|=k} \varphi_{\tau, \mu}\left(t\left(\xi^{\otimes n}\right) t\left(\eta^{\otimes n}\right)^{*}\right) & =\sum_{|\mu|=k} \tau \pi^{-1}\left(t\left(x_{\mu}\right)^{*} t\left(\xi^{\otimes n}\right) t\left(\eta^{\otimes n}\right)^{*} t\left(x_{\mu}\right)\right) \\
& =\sum_{|\mu|=k} \sum_{|\nu|=k-n} \tau \pi^{-1}\left(t\left(x_{\mu}\right)^{*} t\left(\xi^{\otimes n}\right) t\left(x_{\nu}\right) t\left(x_{\nu}\right)^{*} t\left(\eta^{\otimes n}\right)^{*} t\left(x_{\mu}\right)\right) \\
& =\sum_{|\nu|=k-n|\mu|=k} \sum_{\mid \nu \pi^{-1}\left(t\left(x_{\nu}\right)^{*} t\left(\eta^{\otimes n}\right)^{*} t\left(x_{\mu}\right) t\left(x_{\mu}\right)^{*} t\left(\xi^{\otimes n}\right) t\left(x_{\nu}\right)\right)} \\
& =\sum_{|\nu|=k-n} \tau \pi^{-1}\left(t\left(x_{\nu}\right)^{*} t\left(\eta^{\otimes n}\right)^{*} t\left(\xi^{\otimes n}\right) t\left(x_{\nu}\right)\right) \\
& =\sum_{|\nu|=k-n} \varphi_{\tau, \nu}\left(t\left(\eta^{\otimes n}\right)^{*} t\left(\xi^{\otimes n}\right)\right) .
\end{aligned}
$$

Hence we obtain

$$
\begin{aligned}
\varphi_{\tau}\left(t\left(\xi^{\otimes n}\right) t\left(\eta^{\otimes n}\right)^{*}\right) & =c_{\tau, \beta}^{-1} \sum_{k=0}^{\infty} e^{-k \beta} \sum_{|\mu|=k} \varphi_{\tau, \mu}\left(t\left(\xi^{\otimes n}\right) t\left(\eta^{\otimes n}\right)^{*}\right) \\
& =c_{\tau, \beta}^{-1} \sum_{k=n}^{\infty} e^{-k \beta} \sum_{|\mu|=k-n} \varphi_{\tau, \mu}\left(t\left(\eta^{\otimes n}\right)^{*} t\left(\xi^{\otimes n}\right)\right) \\
& =c_{\tau, \beta}^{-1} \sum_{k=0}^{\infty} e^{-(k+n) \beta} \sum_{|\mu|=k} \varphi_{\tau, \mu}\left(t\left(\eta^{\otimes n}\right)^{*} t\left(\xi^{\otimes n}\right)\right) \\
& =c_{\tau, \beta}^{-1} \sum_{k=0}^{\infty} e^{-(k+n) \beta} \sum_{|\mu|=k} \tau\left(\left\langle\eta^{\otimes m} \otimes x_{\mu}, \xi^{\otimes n} \otimes x_{\mu}\right\rangle\right) .
\end{aligned}
$$

We verify that $\varphi_{\tau} \in \mathrm{E}_{\beta}\left(\mathcal{T}_{X}\right)$ by using Proposition 3.1. By definition we have that if $n \neq m$ then $\varphi_{\tau}\left(t\left(\xi^{\otimes n}\right) t\left(\eta^{\otimes m}\right)^{*}\right)=0$. Now if $n=m$ then we directly compute

$$
\begin{aligned}
\varphi_{\tau}\left(t\left(\xi^{\otimes n}\right) t\left(\eta^{\otimes n}\right)^{*}\right) & =c_{\tau, \beta}^{-1} \sum_{k=0}^{\infty} e^{-(k+n) \beta} \sum_{|\mu|=k} \varphi_{\tau, \mu}\left(t\left(\eta^{\otimes n}\right)^{*} t\left(\xi^{\otimes n}\right)\right) \\
& =e^{-n \beta} c_{\tau, \beta}^{-1} \sum_{k=0}^{\infty} e^{-k \beta} \sum_{|\mu|=k} \varphi_{\tau, \mu}\left(t\left(\eta^{\otimes n}\right)^{*} t\left(\xi^{\otimes n}\right)\right) \\
& =e^{-n \beta} \varphi_{\tau}\left(t\left(\eta^{\otimes n}\right)^{*} t\left(\xi^{\otimes n}\right)\right) .
\end{aligned}
$$

In order to show that $\varphi_{\tau} \in \mathrm{E}_{\beta}^{\mathrm{fin}}\left(\mathcal{T}_{X}\right)$ we compute

$$
\begin{aligned}
\sum_{k=0}^{n} \varphi_{\tau}\left(p_{k}\right) & =1-\sum_{|\nu|=n+1} \varphi_{\tau}\left(t\left(x_{\nu}\right) t\left(x_{\nu}\right)^{*}\right) \\
& =1-c_{\tau, \beta}^{-1} \sum_{k=0}^{\infty} e^{-(n+1+k) \beta} \sum_{|\nu|=n+1} \sum_{|\mu|=k} \tau\left(\left\langle x_{\nu} \otimes x_{\mu}, x_{\nu} \otimes x_{\mu}\right\rangle\right) \\
& =c_{\tau, \beta}^{-1} \sum_{k=0}^{n} e^{-k \beta} \sum_{|\mu|=k} \tau\left(\left\langle x_{\mu}, x_{\mu}\right\rangle\right),
\end{aligned}
$$

Applying for $n=0$ yields $\varphi_{\tau}\left(p_{0}\right)=c_{\tau, \beta}^{-1}$. Taking the limit $n \rightarrow \infty$ gives $\sum_{k=0}^{\infty} \varphi_{\tau}\left(p_{k}\right)=c_{\tau, \beta}^{-1} c_{\tau, \beta}=1$, and so $\varphi_{\tau} \in \mathrm{E}_{\beta}^{\mathrm{fin}}\left(\mathcal{T}_{X}\right)$. 
Secondly we show that this correspondence is surjective. To this end fix $\varphi \in \mathrm{E}_{\beta}^{\mathrm{fin}}\left(\mathcal{T}_{X}\right)$. Inequality (4.2) gives that $\varphi\left(p_{0}\right) \neq 0$ and thus we can define the state $\tau_{\varphi}$ on $A$ by

$$
\tau_{\varphi}(a):=\varphi\left(p_{0}\right)^{-1} \varphi\left(p_{0} \pi(a) p_{0}\right) \text { for all } a \in A .
$$

Moreover $\tau_{\varphi}$ is in $\mathrm{T}(A)$ since

$$
\varphi\left(p_{0}\right) \tau_{\varphi}(a b)=\varphi\left(p_{0} \pi(a) \pi(b) p_{0}\right)=\varphi\left(\pi(b) p_{0} \pi(a)\right)=\varphi\left(p_{0} \pi(b) \pi(a) p_{0}\right)=\varphi\left(p_{0}\right) \tau(b a),
$$

where we used that $p_{0} \in \pi(A)^{\prime}$ and $\sigma_{i \beta}(\pi(a))=\pi(a)$. In order to show that $\tau_{\varphi} \in \mathrm{T}_{\beta}(A)$ it suffices to show that

$$
\varphi\left(p_{0}\right)^{-1}=\sum_{k=0}^{\infty} e^{-k \beta} \sum_{|\mu|=k} \tau_{\varphi}\left(\left\langle x_{\mu}, x_{\mu}\right\rangle\right) .
$$

However a direct computation yields

$$
\varphi\left(p_{0}\right) \sum_{|\mu|=k} \tau_{\varphi}\left(\left\langle x_{\mu}, x_{\mu}\right\rangle\right)=\sum_{|\mu|=k} \varphi\left(p_{0} t\left(x_{\mu}\right)^{*} t\left(x_{\mu}\right) p_{0}\right)=e^{k \beta} \sum_{|\mu|=k} \varphi\left(t\left(x_{\mu}\right) p_{0} t\left(x_{\mu}\right)^{*}\right)=e^{k \beta} \varphi\left(p_{k}\right) .
$$

Since $\varphi \in \mathrm{E}_{\beta}^{\mathrm{fin}}\left(\mathcal{T}_{X}\right)$ we have

$$
\sum_{k=0}^{\infty} e^{-k \beta} \sum_{|\mu|=k} \tau_{\varphi}\left(\left\langle x_{\mu}, x_{\mu}\right\rangle\right)=\varphi\left(p_{0}\right)^{-1} \sum_{k=0}^{\infty} \varphi\left(p_{k}\right)=\varphi\left(p_{0}\right)^{-1} .
$$

Surjectivity now follows by showing that $\varphi=\Phi\left(\tau_{\varphi}\right)$. Since both are $(\sigma, \beta)$-KMS states, by Proposition 3.1 it suffices to show that they agree on $\pi(A)$. Since $\varphi$ is implemented by a state on $\mathcal{K}(\mathcal{F} X)$, for every $a \in A$ we have that

$$
\begin{aligned}
\varphi(\pi(a)) & =\lim _{m} \sum_{k, l=0}^{m} \varphi\left(p_{k} \pi(a) p_{l}\right)=\lim _{m} \sum_{k=0}^{m} \varphi\left(p_{k} \pi(a)\right) \\
& =\lim _{m} \sum_{k=0}^{m} \sum_{|\mu|=k} \varphi\left(t\left(x_{\mu}\right) p_{0} t\left(x_{\mu}\right)^{*} \pi(a)\right) \\
& =\lim _{m} \sum_{k=0}^{m} \sum_{|\mu|=m} e^{-k \beta} \varphi\left(p_{0} t\left(x_{\mu}\right)^{*} \pi(a) t\left(x_{\mu}\right) p_{0}\right) \\
& \left.=c_{\tau_{\varphi}, \beta}^{-1} \sum_{k=0}^{\infty} e^{-k \beta} \sum_{|\mu|=k} \tau_{\varphi}\left(\left\langle x_{\mu}, a x_{\mu}\right)\right\rangle\right)=\Phi\left(\tau_{\varphi}\right)(\pi(a)) .
\end{aligned}
$$

To show injectivity let $\tau \in \mathrm{T}_{\beta}(A)$ and use the vector states $\varphi_{\tau, \mu}$ to get

$$
\sum_{|\mu|=k} \varphi_{\tau, \mu}\left(p_{0} \pi(a) p_{0}\right)= \begin{cases}\tau(a) & \text { if } k=0 \\ 0 & \text { otherwise. }\end{cases}
$$

Therefore we have

$$
\varphi_{\tau}\left(p_{0} \pi(a) p_{0}\right)=c_{\tau, \beta}^{-1} \sum_{k=0}^{\infty} e^{-k \beta} \sum_{|\mu|=k} \varphi_{\tau, \mu}\left(p_{0} \pi(a) p_{0}\right)=\varphi_{\tau}\left(p_{0}\right) \tau(a)
$$

showing that $\tau$ is uniquely identified by $\varphi_{\tau}$.

Finally we show that $\Phi^{-1}$ is weak*-continuous. To this end let $\varphi_{j}, \varphi \in \mathrm{E}_{\beta}^{\mathrm{fin}}\left(\mathcal{T}_{X}\right)$ such that $\varphi_{j} \longrightarrow \varphi$ in the weak*-topology. As $\varphi_{j}\left(p_{0}\right) \neq 0$ and $\varphi\left(p_{0}\right) \neq 0$ we get that $\tau_{\varphi_{j}}(a) \longrightarrow \tau_{\varphi}(a)$ for all $a \in A$. Hence $\Phi^{-1}$ is a continuous bijection from the compact space $\mathrm{E}_{\beta}^{\mathrm{fin}}\left(\mathcal{T}_{X}\right)$ onto the Hausdorff space $\mathrm{T}_{\beta}(A)$. Thus if $\mathrm{E}_{\beta}^{\mathrm{fin}}\left(\mathcal{T}_{X}\right)$ is weak*-closed then $\Phi$ is a homeomorphism. 
Corollary 6.2. Let $\Phi: \mathcal{T}_{\beta}(A) \rightarrow \mathrm{E}_{\beta}^{\mathrm{fin}}\left(\mathcal{T}_{X}\right)$ be the map of Theorem 6.1. If $\tau=\lambda \tau_{1}+(1-\lambda) \tau_{2}$ for $\tau_{1}, \tau_{2} \in \mathrm{T}_{\beta}(A)$ and $\lambda \in[0,1]$ then

$$
\Phi(\tau)=\lambda \frac{c_{\tau_{1}, \beta}}{c_{\tau, \beta}} \Phi\left(\tau_{1}\right)+(1-\lambda) \frac{c_{\tau_{2}, \beta}}{c_{\tau, \beta}} \Phi\left(\tau_{2}\right) .
$$

Conversely, if $\varphi=\lambda \varphi_{1}+(1-\lambda) \varphi_{2}$ for $\varphi_{1}, \varphi_{2} \in \mathrm{E}_{\beta}^{\mathrm{fin}}\left(\mathcal{T}_{X}\right)$ and $\lambda \in[0,1]$ then

$$
\Phi^{-1}(\varphi)=\lambda \frac{\varphi_{1}\left(p_{0}\right)}{\varphi\left(p_{0}\right)} \Phi^{-1}\left(\varphi_{1}\right)+(1-\lambda) \frac{\varphi_{2}\left(p_{0}\right)}{\varphi\left(p_{0}\right)} \Phi^{-1}\left(\varphi_{2}\right) .
$$

Consequently, the parametrization $\Phi$ fixes the extreme points.

Proof. For the forward direction it is clear that $c_{\tau, \beta}=\lambda c_{\tau_{1}, \beta}+(1-\lambda) c_{\tau_{2}, \beta}$. Therefore the state

$$
\varphi^{\prime}=\lambda \frac{c_{\tau_{1}, \beta}}{c_{\tau, \beta}} \Phi\left(\tau_{1}\right)+(1-\lambda) \frac{c_{\tau_{2}, \beta}}{c_{\tau, \beta}} \Phi\left(\tau_{2}\right)
$$

is in $\mathrm{E}_{\beta}^{\mathrm{fin}}\left(\mathcal{T}_{X}\right)$ as a convex combination of states in $\mathrm{E}_{\beta}^{\mathrm{fin}}\left(\mathcal{T}_{X}\right)$. Now for every $a \in A$ we have

$$
\begin{aligned}
\varphi_{\tau}(\pi(a)) & =\lambda c_{\tau, \beta}^{-1} \sum_{k=0}^{\infty} e^{-k \beta} \sum_{|\mu|=k} \tau_{1}\left(\left\langle x_{\mu}, a x_{\mu}\right\rangle\right)+(1-\lambda) c_{\tau, \beta}^{-1} \sum_{k=0}^{\infty} e^{-k \beta} \sum_{|\mu|=k} \tau_{2}\left(\left\langle x_{\mu}, a x_{\mu}\right\rangle\right) \\
& =\lambda \frac{c_{\tau_{1}, \beta}}{c_{\tau, \beta}} \Phi\left(\tau_{1}\right)(\pi(a))+(1-\lambda) \frac{c_{\tau_{2}, \beta}}{c_{\tau, \beta}} \Phi\left(\tau_{2}\right)(\pi(a))=\varphi^{\prime}(\pi(a)) .
\end{aligned}
$$

As both $\varphi^{\prime}$ and $\varphi_{\tau}$ are in $\mathrm{E}_{\beta}\left(\mathcal{T}_{X}\right)$, Proposition 3.1 implies that they are equal. For the converse set $\tau_{1}=\Phi^{-1}\left(\varphi_{1}\right), \tau_{2}=\Phi^{-1}\left(\varphi_{2}\right)$ and $\tau=\Phi^{-1}(\varphi)$. Then by construction, for every $a \in A$ we get that

$$
\varphi\left(p_{0}\right) \tau(a)=\lambda \varphi_{1}\left(p_{0} \pi(a) p_{0}\right)+(1-\lambda) \varphi_{2}\left(p_{0} \pi(a) p_{0}\right)=\lambda \varphi_{1}\left(p_{0}\right) \tau_{1}(a)+(1-\lambda) \varphi_{2}\left(p_{0}\right) \tau_{2}(a) .
$$

Applying for $a=1_{A}$ also gives that $\varphi\left(p_{0}\right)=\lambda_{1} \varphi_{1}\left(p_{0}\right)+\lambda_{2} \varphi_{2}\left(p_{0}\right)$. Finally to see that $\Phi$ fixes the extreme points just notice that the $c$-constants are all non-zero and the equations for $\Phi(\tau)$ and $\Phi^{-1}(\varphi)$ are convex combinations of states.

Corollary 6.3. If $X$ is a $C^{*}$-correspondence of finite rank over $A$ then $h_{X} \leq \max \left\{0, h_{X}^{s}\right\}$.

Proof. If $\beta>h_{X}^{s}$ then Proposition 5.7(iii) implies that $\mathrm{T}_{\beta}(A)=\mathrm{T}(A)$. Therefore Theorem 6.1 gives that $\mathrm{E}_{\beta}\left(\mathcal{T}_{X}\right) \neq \emptyset$ and so $h_{X} \leq \beta$.

The gauge action of $\mathcal{T}_{X}$ is inherited by the $J$-relative Cuntz-Pimsner algebras. Thus we can use the previous parametrization for their equilibrium states. For convenience let us write here $(\rho, v)=\left(q_{J} \pi, q_{J} t\right)$ for the faithful representation of $\mathcal{O}(J, X)$ where $q_{J}: \mathcal{T}_{X} \rightarrow \mathcal{O}(J, X)$ is the canonical quotient map. Hence ker $q_{J}$ is the ideal generated by $\pi(a) p_{0}$ for all $a \in J$ when $p_{0} \in \mathcal{T}_{X}$. We will write simply $q$ when $J=A$.

Theorem 6.4. Let $X$ be a $C^{*}$-correspondence of finite rank over $A$ and let $\beta>0$. Suppose that $J \subseteq \phi_{X}^{-1}(\mathcal{K} X)$. Then there is a bijection

$$
\Phi:\left\{\tau \in \mathrm{T}_{\beta}(A)|\tau|_{J}=0\right\} \rightarrow \mathrm{E}_{\beta}^{\mathrm{fin}}(\mathcal{O}(J, X)) .
$$

If $x=\left\{x_{1}, \ldots, x_{d}\right\}$ is a decomposition of the unit then $\Phi$ is given by

$$
\Phi(\tau)\left(v\left(\xi^{\otimes n}\right) v\left(\xi^{\otimes m}\right)^{*}\right)=\delta_{n, m} c_{\tau, \beta}^{-1} \sum_{k=0}^{\infty} e^{-(k+n) \beta} \sum_{|\mu|=k} \tau\left(\left\langle\eta^{\otimes m} \otimes x_{\mu}, \xi^{\otimes n} \otimes x_{\mu}\right\rangle\right)
$$

for all $\xi^{\otimes n} \in X^{\otimes n}$ and $\eta^{\otimes m} \in X^{\otimes m}$. Moreover $\Phi$ satisfies the convex combination of Corollary 6.2 and thus it preserves extreme points. If, in addition, $\mathrm{E}_{\beta}^{\mathrm{fin}}(\mathcal{O}(J, X))$ is weak ${ }^{*}$-closed then $\Phi$ is a weak ${ }^{*}$-homeomorphism between weak*-compact sets. 
Proof. Fix $q_{J}: \mathcal{T}_{X} \rightarrow \mathcal{O}(J, X)$ be the canonical $*$-epimorphism. Then the $\operatorname{ker} q_{J}=\mathcal{K}(\mathcal{F}(X) J)$ is generated by $\pi(a) p_{0}$ for $a \in J$. Let $\tau \in \mathrm{T}_{\beta}(A)$ and fix $\varphi_{\tau}$ be the associated state in $\mathrm{E}_{\beta}^{\text {fin }}\left(\mathcal{T}_{X}\right)$ given by Theorem 6.1. Then we get that $\varphi_{\tau}\left(\pi(a) p_{0}\right)=c_{\tau, \beta}^{-1} \tau(a)$. Therefore, if $\tau$ vanishes on $J$ then $\varphi_{\tau}$ vanishes on $\operatorname{ker} q_{J}$ and so it induces a state on $\mathcal{O}(J, X)$. As the unital quotient map intertwines the gauge actions the induced state is in $\mathrm{E}_{\beta}^{\mathrm{fin}}(\mathcal{O}(J, X))$. Conversely if $\varphi \in \mathrm{E}_{\beta}^{\mathrm{fin}}(\mathcal{O}(J, X))$ then $\bar{\varphi} q_{J} \in \mathrm{E}_{\beta}^{\mathrm{fin}}(\mathcal{T} X)$ and it defines $\tau_{\bar{\varphi}} \in \mathrm{T}_{\beta}(A)$ by Theorem 6.1. By construction $\tau_{\bar{\varphi}}$ vanishes on $J$ as $q_{J}\left(p_{0} \pi(a) p_{0}\right)=q_{J}\left(\pi(a) p_{0}\right)=0$ for all $a \in J$.

\section{The infinite part of the Equilibrium States}

Let us now see how we can parametrize $\mathrm{E}_{\beta}^{\infty}(\mathcal{O}(A, X))$ (and thus all $\mathrm{E}_{\beta}^{\infty}(\mathcal{O}(J, X))$ ). The main point here is that these states are given by extending tracial states on $A$ rather than by taking statistical approximations. When $X$ is non-degenerate and injective then the existence of such a $\Psi$ can be derived by combining [23, Theorem 2.1 and Theorem 2.5]. However the attack therein is essentially different, as the well definedness of the extension is verified by using perturbations of the action. Following [18, Theorem 3.18] we can directly construct the extension within the fixed point algebra (and by keeping the same action).

Theorem 7.1. Let $X$ be a $C^{*}$-correspondence of finite rank over $A$ and let $\beta>0$. Let

$$
I:=\left\{a \in A \mid \lim _{n}\left\|\phi_{X}(a) \otimes \operatorname{id}_{X \otimes n-1}\right\|=0\right\} .
$$

Then there is an affine weak ${ }^{*}$-homeomorphism

$$
\Psi:\left\{\tau \in \operatorname{AVT}_{\beta}(A)|\tau|_{I}=0\right\} \rightarrow \mathrm{E}_{\beta}^{\infty}(\mathcal{O}(A, X)) \text { such that }\left.\Psi(\varphi)\right|_{\rho(A)}=\tau .
$$

In particular $\Psi$ induces an affine weak ${ }^{*}$-homeomorphism onto $\mathrm{E}_{\beta}^{\infty}\left(\mathcal{T}_{X}\right)$.

Proof. By Theorem 4.6, $\mathrm{E}_{\beta}^{\infty}\left(\mathcal{T}_{X}\right)$ consists exactly of the $(\sigma, \beta)$-KMS states that factor through $\mathrm{E}_{\beta}(\mathcal{O}(A, X))$. The ideal $I$ is the kernel of $\left.q\right|_{\pi(A)}$ for the canonical *-epimorphism $q: \mathcal{T}_{X} \rightarrow \mathcal{O}(A, X)$. Suppose first that $X$ is not injective and fix $(\rho, v)$ such that $\mathcal{O}(A, X)=\mathrm{C}^{*}(\rho, v)$. Then $I=\operatorname{ker} \rho$ and we claim that $\mathcal{O}_{X}$ is canonically $*$-isomorphic to $\mathcal{O}_{Y}$ for $Y=v(X)$ and $B=\rho(A)$. To this end first notice that $Y$ is injective and of finite rank so that $J_{Y}=B$. Indeed the covariance gives that

$$
\rho(a)=\rho(a) \sum_{i \in[d]} v\left(x_{i}\right) v\left(x_{i}\right)^{*} \text { for all } a \in A .
$$

Hence if $a+I \in \operatorname{ker} \phi_{Y}$ then $\rho(a)=\rho(a) \sum_{i \in[d]} v\left(x_{i}\right) v\left(x_{i}\right)^{*}=0$, so that $a \in \operatorname{ker} \rho=I$. Secondly it is clear that $\left(\operatorname{id}_{B}, \operatorname{id}_{Y}\right)$ defines a $B$-covariant pair for $Y$ since for $b=\rho(a)$ we have

$$
\psi_{\operatorname{id}_{Y}}\left(\phi_{Y}(b)\right)=\rho(a)=\operatorname{id}_{B}(b) .
$$

Moreover it inherits a gauge action and trivially $\mathrm{id}_{B}$ is injective on $B$. Thus the Gauge-InvariantUniqueness-Theorem asserts that $\mathcal{O}_{Y}=\mathrm{C}^{*}\left(\operatorname{id}_{B}, \operatorname{id}_{v}\right)=\mathrm{C}^{*}(\rho, v)$.

Therefore without loss of generality we may assume that $X$ is injective so that $\mathcal{O}(A, X)=\mathcal{O}_{X}$ and $I=(0)$. We have to produce a weak*-homeomorphism $\Psi: \operatorname{AVT}_{\beta}(A) \rightarrow \mathrm{E}_{\beta}^{\infty}\left(\mathcal{O}_{X}\right)$ such that $\Psi^{-1}(\varphi)=\varphi \rho$. Let $\varphi \in \mathrm{E}_{\beta}^{\infty}\left(\mathcal{O}_{X}\right)$ and set $\tau:=\varphi \rho \in \mathrm{T}(A)$. Therefore the KMS-condition yields

$$
\tau(a)=\varphi\left(\rho(a) \sum_{i \in[d]} v\left(x_{i}\right) v\left(x_{i}\right)^{*}\right)=e^{-\beta} \sum_{i \in[d]} \varphi\left(v\left(x_{i}\right)^{*} \rho(a) v\left(x_{i}\right)\right)=e^{-\beta} \sum_{i \in[d]} \tau\left(\left\langle x_{i}, a x_{i}\right\rangle\right)
$$

and thus $\tau \in \operatorname{AVT}_{\beta}(A)$. Now fix $\tau \in \operatorname{AVT}_{\beta}(A)$ and we will construct a $\varphi_{\tau} \in \mathrm{E}_{\beta}^{\infty}(\mathcal{O}(A, X))$. To this end we use a well known construction, that goes as back as [33]. Namely, when $X$ is injective and the left action is by compacts then the fixed point algebra $\mathcal{O}_{X}$ can be identified with the direct limit

$$
A \stackrel{\phi_{X}}{\longrightarrow} \mathcal{K} X \stackrel{\otimes \mathrm{id}_{X}}{\longrightarrow} \mathcal{K} X^{\otimes 2} \stackrel{\otimes \mathrm{id}_{X}}{\longrightarrow} \cdots
$$

where $\left[\otimes \operatorname{id}_{X}\right](t)=t \otimes \operatorname{id}_{X}$. In our case this identification is given by

$$
\theta_{\xi^{\otimes n}, \eta^{\otimes n}} \otimes \mathrm{id}_{X}=\sum_{i \in[d]} \theta_{\xi^{\otimes n} \otimes x_{i}, \eta^{\otimes n} \otimes x_{i}},
$$


as a direct computation on elementary tensors shows. Therefore $\mathcal{O}_{X}^{\gamma}$ is the inductive limit of the increasing sequence $\left\{\left(\mathcal{O}_{X}^{\gamma}\right)_{n}\right\}_{n \in n \mathbb{N}}$ for

$$
\left(\mathcal{O}_{X}^{\gamma}\right)_{n}:=\overline{\operatorname{span}}\left\{v\left(\xi^{\otimes n}\right) v\left(\eta^{\otimes n}\right)^{*} \mid \xi^{\otimes n}, \eta^{\otimes n} \in X^{\otimes n}\right\}=\psi_{v, n}\left(\mathcal{K} X^{\otimes n}\right)
$$

by writing

$$
v\left(\xi^{\otimes n}\right) v\left(\eta^{\otimes n}\right)^{*}=\sum_{i \in[d]} v\left(\xi^{\otimes n}\right) v\left(x_{i}\right) v\left(x_{i}\right)^{*} v\left(\eta^{\otimes n}\right)^{*} .
$$

We define the functionals $\varphi_{n}$ on $\left(\mathcal{O}_{X}^{\gamma}\right)_{n}$ by

$$
\varphi_{n}\left(\psi_{v, n}\left(k_{n}\right)\right):=e^{-n \beta} \sum_{|\mu|=n} \tau\left(\left\langle x_{\mu}, k_{n} x_{\mu}\right\rangle\right) \text { for } k_{n} \in \mathcal{K} X^{\otimes n} .
$$

To see that it is well defined notice that for a positive $k_{n}$ we have

$$
e^{-n \beta} \sum_{|\mu|=n} \tau\left(\left\langle x_{\mu}, k_{n} x_{\mu}\right\rangle\right) \leq\left\|k_{n}\right\| e^{-n \beta} \sum_{|\mu|=n} \tau\left(\left\langle x_{\mu}, x_{\mu}\right\rangle\right) \leq\left\|k_{n}\right\| .
$$

In particular we have $\varphi_{n}\left(\rho\left(1_{A}\right)\right)=e^{-n \beta} \sum_{|\mu|=n} \tau\left(\left\langle x_{\mu}, x_{\mu}\right\rangle\right)=1$ and so each $\varphi_{n}$ is a state. By construction we have that

$$
\begin{aligned}
\varphi_{n}\left(\psi_{v, n}\left(\theta_{\xi^{\otimes n}, \eta^{\otimes n}}\right)\right) & =e^{-n \beta} \sum_{|\mu|=n} \tau\left(\left\langle x_{\mu}, \xi^{\otimes n}\right\rangle\left\langle\eta^{\otimes n}, x_{\mu}\right\rangle\right) \\
& =e^{-n \beta} \sum_{|\mu|=n} \tau\left(\left\langle\eta^{\otimes n}, x_{\mu}\right\rangle\left\langle x_{\mu}, \xi^{\otimes n}\right\rangle\right)=e^{-n \beta} \tau\left(\left\langle\eta^{\otimes n}, \xi^{\otimes n}\right\rangle\right) .
\end{aligned}
$$

We see that the collection $\left\{\varphi_{n} \mid n \in \mathbb{N}\right\}$ is compatible with the direct limit structure since

$$
\begin{aligned}
\varphi_{n+1}\left(\psi_{v, n+1}\left(\theta_{\xi^{\otimes n}, \eta^{\otimes n}} \otimes \mathrm{id}_{X}\right)\right) & =\sum_{i \in[d]} \varphi_{n+1}\left(v\left(\xi^{\otimes n}\right) v\left(x_{i}\right) v\left(x_{i}\right)^{*} v\left(\eta^{\otimes n}\right)^{*}\right) \\
& =e^{-(n+1) \beta} \sum_{|\mu|=n+1} \sum_{i \in[d]} \tau\left(\left\langle x_{\mu}, \xi^{\otimes n} \otimes x_{i}\right\rangle\left\langle\eta^{\otimes n} \otimes x_{i}, x_{\mu}\right\rangle\right) \\
& =e^{-(n+1) \beta} \sum_{i \in[d]} \sum_{|\mu|=n+1} \tau\left(\left\langle\eta^{\otimes n} \otimes x_{i}, x_{\mu}\right\rangle\left\langle x_{\mu}, \xi^{\otimes n} \otimes x_{i}\right\rangle\right) \\
& =e^{-(n+1) \beta} \sum_{i \in[d]} \tau\left(\left\langle x_{i},\left\langle\eta^{\otimes n}, \xi^{\otimes n}\right\rangle x_{i}\right\rangle\right) \\
& =e^{-n \beta} \tau\left(\left\langle\eta^{\otimes n}, \xi^{\otimes n}\right\rangle\right)=\varphi_{n}\left(\psi_{v, n}\left(\theta_{\xi^{\otimes n}, \eta^{\otimes n}}\right)\right) .
\end{aligned}
$$

Therefore it defines a state $\varphi_{\tau}$ in the limit which extends $\tau$ such that

$$
\varphi_{\tau}\left(v\left(\xi^{\otimes n}\right) v\left(\eta^{\otimes n}\right)^{*}\right)=\varphi_{n}\left(v\left(\xi^{\otimes n}\right) v\left(\eta^{\otimes n}\right)^{*}\right)=e^{-n \beta} \tau\left(\left\langle\eta^{\otimes n}, \xi^{\otimes n}\right\rangle\right) .
$$

Let $E: \mathcal{O}_{X} \rightarrow \mathcal{O}_{X}^{\gamma}$ be the conditional expectation coming from the gauge action. Then Proposition 3.1 yields that the induced state $\varphi_{\tau} E$ is a $(\sigma, \beta)$-KMS state on $\mathcal{O}_{X}$. The same proposition implies that $\varphi_{\tau} E$ is the unique $(\sigma, \beta)$-KMS state with restriction $\tau$ on $A$. Therefore $\Psi$ is injective.

It is immediate that $\Psi^{-1}$ is weak*-continuous and affine. Since $\mathrm{E}_{\beta}^{\infty}(\mathcal{O}(A, X))$ is weak ${ }^{*}$-compact and $\operatorname{AVT}_{\beta}(A)$ is Hausdorff, it follows that $\Psi$ is a weak*-homeomorphism.

The same method applies to parametrize the KMS-states at $\beta=0$, i.e., the gauge-invariant tracial states on $\mathcal{O}(A, X)$. However we cannot have arbitrarily large $\beta>0$ for $\mathcal{O}(A, X)$. Obviously $q\left(p_{0}\right)=0$ and so $\mathrm{E}_{\beta}(\mathcal{O}(A, X))=\mathrm{E}_{\beta}^{\infty}(\mathcal{O}(A, X))$. Therefore Theorem 6.4 is void for $\mathcal{O}(A, X)$ at $\beta \leq h_{X}^{s}$; and there is a good reason for this.

Proposition 7.2. If $X$ is a $C^{*}$-correspondence of finite rank over $A$ then $\mathrm{E}_{\beta}(\mathcal{O}(A, X))=\emptyset$ for all $\beta>h_{X}^{s}$. If, in addition, $A$ is abelian and $h_{X}^{s}>0$ then $\mathcal{O}(A, X)$ attains equilibrium states at $h_{X}^{s}$.

Proof. Since $\varphi q \in \mathrm{E}_{\beta}^{\infty}\left(\mathcal{T}_{X}\right)$ for $\varphi \in \mathrm{E}_{\beta}(\mathcal{O}(A, X))$, Proposition 5.7 yields $\beta \leq h_{X}^{s}$. The same proposition and Theorem 7.1 gives the second part of the statement. 
Corollary 7.3. Let $X$ be a $C^{*}$-correspondence of finite rank over $A$. Then:

(i) $h_{X}=\max \left\{0, \inf \left\{h_{X}^{\tau} \mid \tau \in \mathrm{T}(A)\right\}\right\}$.

(ii) If $0<h_{X}$, or if $0 \leq h_{\tau}$ for all $\tau \in \mathrm{T}(A)$, then $h_{X}=\min \left\{h_{X}^{\tau} \mid \tau \in \mathrm{T}(A)\right\}$.

(iii) If $h_{X}^{\tau}=h_{X}^{s}$ for all $\tau \in \mathrm{T}(A)$ then $\mathrm{E}_{\beta}^{\infty}\left(\mathcal{T}_{X}\right)=\emptyset$ for all $\beta>h_{X}$.

Proof. For item (i) let $\beta \geq h_{X}$ so that $\mathrm{E}_{\beta}\left(\mathcal{T}_{X}\right) \neq \emptyset$. Due to the decomposition and the parametrization we get that $\mathrm{T}_{\beta}(A) \neq \emptyset$ or $\operatorname{AVT}_{\beta}(A) \neq \emptyset$. In any case there is a $\tau \in \mathrm{T}(A)$ such that $h_{X}^{\tau} \leq \beta$. Therefore

$$
\inf \left\{h_{X}^{\tau} \mid \tau \in \mathrm{T}(A)\right\} \leq h_{X} .
$$

Suppose there were a $\tau \in \mathrm{T}(A)$ such that $h_{X}^{\tau}<h_{X}$. If $h_{X}^{\tau}<0$ then it is clear that $\tau \in \mathrm{T}_{\beta}(A)$ for all $\beta>0$ in which case $h_{X}=0$. If $h_{X}^{\tau}>0$ then choose $\beta \in\left(h_{X}^{\tau}, h_{X}\right)$. Then the root test gives that $c_{\tau, \beta}<\infty$ and thus the contradiction $\mathrm{E}_{\beta}^{\mathrm{fin}}\left(\mathcal{T}_{X}\right) \neq \emptyset$. For item (ii), weak*-compactness gives that $\mathrm{E}_{h_{X}}\left(\mathcal{T}_{X}\right) \neq \emptyset$. We consider two possible cases for $h_{X}$ :

Case (a). If $h_{X}>0$ then item (i) implies that $h_{X}=\inf \left\{h_{X}^{\tau} \mid \tau \in \mathrm{T}(A)\right\}$. Now we can decompose a $\varphi \in \mathrm{E}_{h_{X}}\left(\mathcal{T}_{X}\right)$ and use the parametrization of each component to get a $\tau_{0} \in \mathrm{T}_{h_{X}}(A) \cup \operatorname{AVT}_{h_{X}}(A)$ with $0 \leq h_{X}^{\tau_{0}} \leq h_{X}$. However by item (i) we have that $h_{X}^{\tau_{0}} \geq h_{X}$ and thus we have equality, i.e., a minimum at $h_{X}^{\tau_{0}}$.

Case (b). If $h_{X}=0$ but $h_{X}^{\tau} \geq 0$ for all $\tau \in \mathrm{T}(A)$, then $\mathcal{T}_{X}$ admits a tracial state $\varphi$ such that

$$
\sum_{|\mu|=k} \varphi \pi\left(\left\langle x_{\mu}, x_{\mu}\right\rangle\right)=\varphi\left(\sum_{|\mu|=k} t\left(x_{\mu}\right) t\left(x_{\mu}\right)^{*}\right) \leq \varphi\left(\pi\left(1_{A}\right)\right)=1 .
$$

As this holds for all $k \in \mathbb{Z}_{+}$we have that $0 \leq h_{X}^{\tau_{0}} \leq \log 1$ and so $h_{X}^{\tau_{0}}=0=h_{X}$ for $\tau_{0}:=\varphi \pi \in \mathrm{T}(A)$. The third item follows by Proposition 7.2.

\section{Comments and APplications}

8.1. Unit decompositions. The strong entropy requires taking the infimum over all possible unit decompositions. This is because the notion of basis is not well defined for $\mathrm{C}^{*}$-correspondences over non-commutative $\mathrm{C}^{*}$-algebras. Let us give such an example here.

Example 8.1. Let $A=C(K) \oplus \mathcal{O}_{2}$ for a compact and Hausdorff space $K$ and the Cuntz algebra $\mathcal{O}_{2}=\mathrm{C}^{*}\left(s_{1}, s_{2}\right)$. Let $\alpha \in \operatorname{End}(A)$ be given by $\alpha(a, b)=\left(a, s_{1} b s_{1}^{*}+s_{2} b s_{2}^{*}\right)$ and let $X$ be the induced $\mathrm{C}^{*}$-correspondence ${ }_{\alpha} A$. That is $X=A$ as a vector space and

$$
\langle\xi, \eta\rangle=\xi^{*} \eta \quad \text { and } \quad(a, b) \cdot \xi \cdot(c, d)=\alpha(a, b) \xi(c, d) .
$$

We chose $A$ to have a commutative part so that $\mathrm{T}(A) \neq \emptyset$. In $[\mathbf{2 0}]$ it is shown that $\emptyset \neq \mathrm{E}_{\beta}\left(\mathcal{T}_{X}\right)=$ $\mathrm{E}_{\beta}^{\mathrm{fin}}\left(\mathcal{T}_{X}\right)$ for all $\beta \in(0, \infty)$.

Now ${ }_{\alpha} A$ admits at least two unit decompositions $x=\{(1,1)\}$ and $y=\left\{\left(1, s_{1}\right),\left(1, s_{2}\right)\right\}$. It is clear that $\sum_{|\mu|=k}\left\langle x_{\mu}, x_{\mu}\right\rangle=1$. On the other hand we have that $y_{1}=\left(1, s_{1}\right)$ and $y_{2}=\left(1, s_{2}\right)$ are orthonormal and so $\left\langle y_{\mu}, y_{\mu}\right\rangle=(1,1)$ for all $\mu \in \mathbb{F}_{+}^{2}$. We then see that they have different entropies as

$$
h_{X}^{x}=\limsup _{k} \frac{1}{k} \log \left\|\sum_{|\mu|=k}\left\langle x_{\mu}, x_{\mu}\right\rangle\right\|=0<\log 2=\limsup _{k} \frac{1}{k} \log \left\|\sum_{|\mu|=k}\left\langle y_{\mu}, y_{\mu}\right\rangle\right\|=h_{X}^{y} .
$$

8.2. Orthogonal bases. In several examples, the $\mathrm{C}^{*}$-correspondence is over an abelian $A$ and admits a finite orthonormal basis. From our analysis, and in particular from Corollary 7.3, we get directly the KMS-structure in these cases:

(i) $h_{X}^{\tau}=\log d$ for all $\tau \in \mathrm{T}(A)$ and $h_{X}=h_{X}^{s}=\log d$;

(ii) $\mathrm{E}_{\beta}\left(\mathcal{T}_{X}\right)=\mathrm{E}_{\beta}^{\mathrm{fin}}\left(\mathcal{T}_{X}\right) \neq \emptyset$ for all $\beta>\log d$, and $\mathrm{E}_{\log d}\left(\mathcal{T}_{X}\right)=\mathrm{E}_{\log d}^{\infty}\left(\mathcal{T}_{X}\right) \neq \emptyset$.

Indeed suppose that $X$ admits a finite orthonormal basis $x=\left\{x_{1}, \ldots, x_{d}\right\}$, i.e., $\left\langle x_{i}, x_{j}\right\rangle=\delta_{i, j}$. Then $\left\langle x_{\mu}, x_{\nu}\right\rangle=\delta_{\mu, \nu}$ when $|\mu|=|\nu|$, so that

$$
h_{X}^{\tau}=h_{X}^{s}=\log d \text { for all } \tau \in \mathrm{T}(A) .
$$


Corollary 7.3 yields that $h_{X}=\log d$ and thus $\mathrm{E}_{\beta}\left(\mathcal{T}_{X}\right)=\mathrm{E}_{\beta}^{\text {fin }}\left(\mathcal{T}_{X}\right)$ for all $\beta>\log d$. Moreover we see that $c_{\tau, \log d}=\sum_{k=0}^{\infty} 1$ for all $\tau \in \mathrm{T}(A)$, so that

$$
\mathrm{E}_{\log d}\left(\mathcal{T}_{X}\right)=\mathrm{E}_{\log d}^{\infty}\left(\mathcal{T}_{X}\right)
$$

As we noted in Proposition 7.2 we have that $\mathrm{E}_{\log d}^{\infty}\left(\mathcal{T}_{X}\right) \neq \emptyset$. As applications we get the full KMS-structure for the Pimsner algebras:

(a) In [20], by applying for $d$ the multiplicity of the dynamical system;

(b) In [26], by applying for $d=|\operatorname{det} A|$ and using [12, Lemma 2.6];

(c) In [27], by applying for $d=|X|$ and using [27, Equation 3.1].

We will see below that Corollary 7.3 gives also the KMS-structure of $[\mathbf{1 4}, \mathbf{1 8}]$ for $\mathrm{C}^{*}$-algebras of irreducible graphs. Notice that in addition to that we provide a clear parametrization of all the equilibrium states at the critical temperature $\beta=\log d$.

8.3. Irreducible graphs. $\mathrm{C}^{*}$-correspondences of finite graphs is the first step away from orthonormal bases. Their KMS-structure gives a nice mixing of cases to distinguish between entropies, i.e.,

(i) It may be the case that $h_{X}<h_{X}^{s}$, and in particular $\mathrm{E}_{\beta}^{\mathrm{fin}}\left(\mathcal{T}_{X}\right) \neq \emptyset$ for every $\beta \in\left(h_{X}, h_{X}^{s}\right)$.

(ii) It may be the case that $\mathrm{E}_{\beta}^{\mathrm{fin}}\left(\mathcal{T}_{X}\right) \neq \emptyset$ and $\mathrm{E}_{\beta}^{\infty}\left(\mathcal{T}_{X}\right) \neq \emptyset$ for some $\beta>0$.

(iii) It may be the case that $\mathrm{E}_{\beta}^{\mathrm{fin}}\left(\mathcal{O}_{X}\right) \neq \emptyset$ at every $\beta>0$.

The equilibrium states of this category have been extensively investigated in $[\mathbf{1 4}, \mathbf{1 5}, \mathbf{1 8}]$. Pimsner algebras of irreducible graphs had been considered in [14] were the Perron-Fröbenius Theorem is used in an essential way. We will see here how the entropy theory we have developed applies and recovers the results therein.

To fix notation, the $\mathrm{C}^{*}$-correspondence $X \equiv X_{G}$ of a graph $G=\left(G^{(0)}, G^{(1)}, s, r\right)$ is the linear span of $\left\{x_{e} \mid e \in G^{(1)}\right\}$ over the abelian $\mathrm{C}^{*}$-algebra generated by orthogonal projections $\left\{p_{v} \mid v \in\right.$ $\left.G^{(0)}\right\}$ such that

$$
\left\langle x_{e}, x_{f}\right\rangle=\delta_{e, f} p_{s(f)} \quad p_{v} x_{e}=\delta_{v, r(e)} x_{e} \quad x_{e} p_{v}=\delta_{s(e), v} x_{e} .
$$

Then $X$ admits the unit decomposition given by the basis $\left\{x_{e} \mid e \in G^{(1)}\right\}$. We will write

$$
\left\{p_{v} \mid v \in \mathcal{G}^{(0)}\right\} \quad \text { and } \quad\left\{L_{e} \mid e \in G^{(1)}\right\}
$$

for the induced Toeplitz-Cuntz-Krieger family, and

$$
\left\{p_{v} \mid v \in G^{(0)}\right\} \quad \text { and } \quad\left\{S_{e} \mid e \in G^{(1)}\right\}
$$

for the induced Cuntz-Krieger family. We use the same symbol for the projections as they have an injective copy in $\mathcal{T}_{X}$ and $\mathcal{O}_{X}=\mathrm{C}^{*}(G)$.

In what follows we write $G$ for both the graph and its adjacency matrix with the understanding that $G_{i j}$ denotes the edges from $i$ to $j$. We will be loose in differentiating between the entry $i$ and the vertex $v_{i}$ corresponding to it. Let us start with an auxiliary proposition.

Proposition 8.2. Let $G$ be a finite non-zero irreducible graph and $p>0$. Then for every $v_{r} \in G$ we have

$$
\limsup _{k} \frac{1}{k} \log \left(p \sum_{j \in G}\left(G^{k}\right)_{r j}\right)=\log \lambda_{G},
$$

where $\lambda_{G}$ is the Perron-Fröbenius eigenvalue of $G$.

Proof. Suppose that $G$ has $n$ vertices and let $w=\left[w_{1}, \ldots, w_{n}\right]$ be the positive Perron-Fröbenius eigenvector corresponding to $\lambda_{G}$. Without loss of generality assume that $w_{1}=\max \left\{w_{1}, \ldots, w_{n}\right\}$ and compute

$$
p \sum_{j \in G}\left(G^{k}\right)_{r j} \geq \frac{p}{w_{1}} \sum_{j \in G}\left(G^{k}\right)_{r j} w_{j}=\frac{p}{w_{1}} \lambda_{G}^{k} w_{r}=\frac{p \cdot w_{r}}{w_{1}} \lambda_{G}^{k} .
$$

On the other hand we have that

$$
p \sum_{j \in G}\left(G^{k}\right)_{r j} \leq p \sum_{i, j \in G}\left(G^{k}\right)_{i j} .
$$


Therefore

$$
\begin{aligned}
\log \lambda_{G} & =\underset{k}{\limsup } \frac{1}{k} \log \left(\frac{p \cdot w_{r}}{w_{1}} \lambda_{G}^{k}\right) \leq \underset{k}{\limsup } \frac{1}{k} \log \left(p \sum_{j \in G}\left(G^{k}\right)_{r j}\right) \\
& \leq \underset{k}{\limsup } \frac{1}{k} \log \left(p \sum_{i, j \in G}\left(G^{k}\right)_{i j}\right)=\limsup _{k} \frac{1}{k} \log \left(\sum_{i, j \in G}\left(G^{k}\right)_{i j}\right)=\log \lambda_{G}
\end{aligned}
$$

where the last equality follows from the Perron-Fröbenius Theorem.

Theorem 8.3. [14] Let $X$ be the $C^{*}$-correspondence associated to a finite non-zero irreducible graph $G$. Let $\lambda_{G}$ be the Perron-Fröbenius eigenvalue of $G$ (and thus of its transpose $G^{t}$ ). Then:

(i) $h_{X}=\log \lambda_{G}$;

(ii) $\mathrm{E}_{\beta}\left(\mathcal{T}_{X}\right)=\mathrm{E}_{\beta}^{\text {fin }}\left(\mathcal{T}_{X}\right)$ for all $\beta>h_{X}$;

(iii) $\mathrm{E}_{h_{X}}\left(\mathcal{T}_{X}\right)=\mathrm{E}_{h_{X}}^{\infty}\left(\mathcal{T}_{X}\right)$ is a singleton on the Perron-Fröbenius eigenvector of $G^{t}$.

Proof. Let $\tau \in \mathrm{T}(A)$. Then by definition we have that

$$
h_{X}^{s} \leq \limsup _{k} \frac{1}{k} \log \left(\sum_{|\mu|=k}\left\|\left\langle x_{\mu}, x_{\mu}\right\rangle\right\|\right)=\limsup _{k} \frac{1}{k} \log \left(\sum_{i, j \in G}\left(G^{k}\right)_{i j}\right)=\log \lambda_{G},
$$

by the Perron-Fröbenius Theorem. On the other hand set

$$
P:=\operatorname{diag}\left\{p_{i} \mid i \in G\right\} \text { where } p_{i}:=\tau\left(p_{v_{i}}\right) .
$$

That is $p_{i}$ is the evaluation of $\tau$ at the projection corresponding to $v_{i}$. Thus $P$ is a diagonal matrix whose entries $\left\{p_{i} \mid i \in G\right\}$ sum up to one. Let a $v_{r} \in G$ such that $p_{r} \neq 0$. Let $w=$ $\left[w_{1}, \ldots, w_{n}\right]$ be the Perron-Fröbenius eigenvector and suppose without loss of generality that $w_{1}=$ $\max \left\{w_{1}, \ldots, w_{n}\right\}$. As in Proposition 8.2 we get

$$
\sum_{|\mu|=k} \tau\left(\left\langle x_{\mu}, x_{\mu}\right\rangle\right)=\sum_{i, j \in G}\left(P G^{k}\right)_{i j} \geq p_{r} \sum_{j \in G}\left(G^{k}\right)_{r j} \geq \frac{p_{r}}{w_{1}} \sum_{j \in G}\left(G^{k}\right)_{r j} w_{j}=\frac{p_{r} \cdot w_{r}}{w_{1}} \lambda_{G}^{k} .
$$

Therefore $h_{X}^{\tau} \geq \log \lambda_{G}$ and so

$$
\log \lambda_{G} \leq h_{X}^{\tau} \leq h_{X}^{s} \leq \log \lambda_{G}
$$

Then Corollary 7.3(iii) gives items (i) and (ii). We also see that

$$
c_{\tau, \log \lambda_{G}} \geq \frac{p_{r} \cdot w_{r}}{w_{1}} \sum_{k=0}^{\infty} e^{-k \log \lambda_{G}} \lambda_{G}^{k}=\infty
$$

so that $\mathrm{T}_{\log \lambda_{G}}(A)=\emptyset$. Hence $\mathrm{E}_{\log \lambda_{G}}\left(\mathcal{T}_{X}\right)=\mathrm{E}_{\log \lambda_{G}}^{\infty}\left(\mathcal{T}_{X}\right)$ and for item (iii) it remains to show that $\mathrm{AVT}_{\log \lambda_{G}}(A)$ is a singleton. A direct computation gives that

$$
\sum_{e \in G^{(1)}} \tau\left(\left\langle x_{e}, p_{i} x_{e}\right\rangle\right)=\sum_{e \in r^{-1}\left(v_{i}\right)} \tau\left(\left\langle x_{e}, x_{e}\right\rangle\right)=\sum_{e \in r^{-1}\left(v_{i}\right)} \tau\left(p_{s(e)}\right)=\sum_{j \in G} g_{j i} p_{j}=\sum_{j \in G^{t}}\left(G^{t}\right)_{i j} p_{j}
$$

Thus $\tau \in \operatorname{AVT}_{\beta}(A)$ if and only if $\left[p_{1}, \ldots, p_{n}\right]$ is an $e^{\beta}$-eigenvector of $G^{t}$ with $\ell^{1}$-norm equal to one. By uniqueness of the Perron-Fröbenius eigenvector we derive that there is only one $\tau \in$ $\mathrm{AVT}_{\log \lambda_{G}}(A)$.

Remark 8.4. The class of irreducible graphs showcases why the convex decomposition between the finite and the infinite part is not weak*-continuous with respect to $\beta>0$. For let $\tau$ be the trace corresponding to the Perron-Fröbenius eigenvalue $\lambda_{G}$. Then it defines a finite state for every $\beta>\log \lambda_{G}$. Letting $\beta \rightarrow \log \lambda_{G}$ gives the infinite KMS-state at $\log \lambda_{G}$. 
8.4. Reducible graphs. Let us now show how the entropy theory can recover the results of [15]. By removing sources one at a time we can write the adjacency matrix of a graph $G$ as an upper triangular matrix

$$
G=\left[\begin{array}{cccc}
G_{1} & * & \cdots & * \\
0 & G_{2} & \cdots & * \\
\vdots & \vdots & \vdots & \vdots \\
0 & 0 & \cdots & G_{m}
\end{array}\right]
$$

where the $G_{1}, \ldots, G_{m}$ are its irreducible components (including some be possibly equal to [0]). It is well known that the entropy of the graph $G$ can be given by the Perron-Fröbenius eigenvalues of the irreducible components in the sense that

$$
h_{G}:=\limsup _{k} \frac{1}{k} \log \left(\sum_{i, j \in G}\left(G^{k}\right)_{i j}\right)=\max \left\{\log \lambda_{G_{1}}, \ldots, \log \lambda_{G_{m}}\right\} ;
$$

see for example [28, Theorem 4.4.4].

Definition 8.5. We say that a component of $G$ is communicated by a $v \in G$ if there is a path from $v$ inside that component. We say that $G_{r}$ is communicated by $G_{s}$ if there exists a path starting at some $v_{s} \in G_{s}$ that ends in some $v_{r} \in G_{r}$.

The proof of the following proposition uses the main idea of the proof of [28, Theorem 4.4.4]. We say that a trace $\tau$ has support in $G_{s}$ if there is a vertex $v \in G_{s}$ such that $\tau\left(p_{v}\right) \neq 0$.

Proposition 8.6. Let $G$ be a finite graph with irreducible components $G_{1}, \ldots, G_{m}$. Then for every $\tau \in \mathrm{T}(A)$ we have that

$$
h_{X}^{\tau}=\max \left\{\log \lambda_{G_{s}} \mid G_{s} \text { is communicated by some } v_{r} \text { in the support of } \tau\right\} .
$$

Proof. For convenience let us set

$$
\lambda=\max \left\{\lambda_{G_{s}} \mid G_{s} \text { is communicated by some } v_{r} \text { in the support of } \tau\right\} .
$$

Let $G_{s}$ be an irreducible component with which a vertex $v_{r}$ in the support of $\tau$ communicates. Thus there is a path of length $N_{0}$ from $v_{r}$ to some $v_{i_{s}} \in G_{s}$. Suppose that $G_{s} \neq[0]$. Then the paths of length $k+N_{0}$ emitting from $v_{r}$ are more than the paths of length $k$ which start at $v_{i_{s}}$ and end inside $G_{s}$. Thus we have

$$
\sum_{i, j \in G}\left(P G^{k+N_{0}}\right)_{i j} \geq p_{r} \sum_{j \in G}\left(G^{k+N_{0}}\right)_{r j} \geq p_{r} \sum_{j \in G_{s}}\left(G_{s}^{k}\right)_{i_{s} j} .
$$

Proposition 8.2 applied for $v_{i_{s}} \in G_{s}$ and $p=p_{r}$ yields

$$
\begin{aligned}
h_{X}^{\tau} & =\underset{k}{\limsup } \frac{1}{k+N_{0}} \log \left(\sum_{i, j \in G}\left(P G^{k+N_{0}}\right)_{i j}\right) \\
& \geq \limsup _{k} \frac{k}{k+N_{0}} \cdot \frac{1}{k} \log \left(p_{r} \sum_{j \in G_{s}}\left(G_{s}^{k}\right)_{i_{s} j}\right)=\log \lambda_{G_{s}} .
\end{aligned}
$$

The same holds trivially if $G_{s}=[0]$. Therefore $h_{X}^{\tau} \geq \log \lambda$.

On the other hand recall that the proof of Perron-Fröbenius Theorem asserts that there exists an $\alpha_{1}>0$ such that

$$
\sum_{i, j \in G_{1}}\left(G_{1}^{k}\right)_{i j} \leq \alpha_{1} \lambda_{G_{1}}^{k}
$$

see for example the comments preceding [28, Proposition 4.2.1]. Likewise for $G_{2}, \ldots, G_{m}$ and set

$$
\alpha:=\max \left\{\alpha_{1}, \ldots, \alpha_{m}\right\} .
$$

First let $v_{r}$ be in the support of $\tau$ and we want to estimate from above the number $\sum_{j \in G}\left(G^{k}\right)_{r j}$ of paths $\mu$ starting at $v_{r}$ with length $k$. Such a path is of the form (read from left to right)

$$
\mu=v_{r} \mu_{1} e_{1} \cdots e_{q} \mu_{q} \text { with }\left|\mu_{1}\right|+\cdots+\left|\mu_{q}\right|+q=k,
$$


with $\mu_{1}, \ldots, \mu_{q}$ be paths entirely in some irreducible components $G_{s_{1}}, \ldots, G_{s_{q}}$, respectively, while the $e_{1}, \ldots, e_{q}$ are transitional edges between different components. Of course $v_{r}$ communicates with all these components so that $\lambda \geq \lambda_{G_{s_{1}}}, \ldots, \lambda_{G_{s_{q}}}$. It is clear that the number of the transitional edges in $\mu$ cannot be more than the number of the components, that is

$$
0 \leq q \leq m \text {. }
$$

Set $M$ be the number of all transitional edges in $G$. For each of the $e_{1}, \ldots, e_{q}$ we have at most $M$ choices and at most $k$ places to insert it. Hence the choices for all the $e_{1}, \ldots, e_{q}$ in $\mu$ cannot exceed $(M k)^{q}$. On the other hand suppose that $\mu_{1}$ has length $k\left(s_{1}\right)$. We have at most $\sum_{i, j \in G_{s_{1}}}\left(G_{s_{1}}^{k}\right)_{i j}$ choices for such a path, and

$$
\sum_{i, j \in G_{s_{1}}}\left(G_{s_{1}}^{k}\right)_{i j} \leq \alpha_{1} \cdot \lambda_{G_{1}}^{k\left(s_{1}\right)} \leq \alpha \cdot \lambda^{k\left(s_{1}\right)} .
$$

Likewise for $G_{s_{2}}, \ldots, G_{s_{q}}$ and we note that

$$
k\left(s_{1}\right)+k\left(s_{2}\right)+\cdots+k\left(s_{q}\right) \leq k .
$$

Therefore we have that

$$
\sum_{j \in G}\left(G^{k}\right)_{r j} \leq(M k)^{q} \cdot\left(\alpha \cdot \lambda^{k\left(s_{1}\right)}\right) \cdots\left(\alpha \cdot \lambda^{k\left(s_{q}\right)}\right) \leq(\alpha M k)^{q} \cdot \lambda^{k} \leq(\alpha M k)^{m} \cdot \lambda^{k} .
$$

Applying for all $v_{r_{1}}, \ldots, v_{r_{\ell}}$ in the support of $\tau$ we get that

$$
\sum_{i, j \in G}\left(P G^{k}\right)_{i j}=\sum_{i=r_{1}}^{r_{\ell}} p_{i} \sum_{j \in G}\left(G^{k}\right)_{i j} \leq \ell \cdot(\alpha M k)^{m} \cdot \lambda^{k} .
$$

Consequently we get that $h_{X}^{\tau} \leq \log \lambda$ and the proof is complete.

We can further identify the tracial components required for the KMS-structure. Note that the root test is always conclusive for those.

Corollary 8.7. Let $G$ be a finite graph and $\beta>0$. Then we have that

$$
\mathrm{T}_{\beta}(A)=\left\{\tau \in \mathrm{T}(A) \mid h_{X}^{\tau}<\beta\right\} \quad \text { and } \quad \operatorname{AVT}_{\beta}(A)=\left\{\tau \in \mathrm{T}(A) \mid G^{t} \tau=e^{\beta} \tau\right\} .
$$

Proof. The part on averaging traces follows from the same computation as in equation (8.1). For $\mathrm{T}_{\beta}(A)$ it suffices to show that $c_{\tau, \beta}<\infty$ if and only if $h_{X}^{\tau}<\beta$. To this end suppose that $\tau$ is supported on the vertices $v_{r_{1}}, \ldots, v_{r_{\ell}}$. For the associated diagonal matrix $P$ set

$$
p_{\min }:=\min \left\{p_{r_{1}}, \ldots, p_{r_{\ell}}\right\} \text {. }
$$

Let $G_{s_{1}}, \ldots, G_{s_{q}}$ be all the irreducible components of $G$ with which a vertex from the support of $\tau$ communicates, and set $G_{s_{0}}$ for the one that corresponds to

$$
\lambda_{G_{s_{0}}}=\max \left\{\lambda_{G_{s_{1}}}, \ldots, \lambda_{G_{s_{q}}}\right\} .
$$

By Proposition 8.6 we have that $h_{X}^{\tau}=\log \lambda_{G_{s_{0}}}$. As before there exist $\alpha_{s}, \alpha_{s}^{\prime}>0$ such that

$$
\alpha_{s}^{\prime} \lambda_{G_{s}}^{k} \leq \sum_{j \in G_{s}}\left(G_{s}^{k}\right)_{i j} \leq \alpha_{s} \lambda_{G_{s}}^{k} .
$$

Set

$$
\alpha^{\prime}=\min \left\{\alpha_{s_{1}}^{\prime}, \ldots, \alpha_{s_{q}}^{\prime}\right\} \quad \text { and } \quad \alpha=\max \left\{\alpha_{s_{1}}, \ldots, \alpha_{s_{q}}\right\} .
$$

If all components $G_{s_{1}}, \ldots, G_{s_{q}}$ are zero then the paths emitting from the support of $\tau$ are only on transitional edges. As in the proof of Proposition 8.6 we get that $\sum_{|\mu|=k} \tau\left(\left\langle x_{\mu}, x_{\mu}\right\rangle\right) \leq(M k)^{m}$ for the total number of transitional edges $M$. Then $h_{X}^{\tau} \leq 0$ and trivially $h_{X}^{\tau}<\beta$.

So suppose there exists at least one component which is not zero. Then $G_{s_{0}} \neq[0]$ with $\lambda_{G_{s_{0}}} \geq 1$. Let $v_{r}$ be in the support of $\tau$ that communicates with some $v_{s_{0}} \in G_{s_{0}}$. As in Proposition 8.6, after some finite step $N_{0}$ we get

$$
\sum_{i, j \in G}\left(P G^{k}\right)_{i j} \geq p_{r} \sum_{j \in G_{s_{0}}}\left(G_{s_{0}}^{k-N_{0}}\right)_{s_{0} j} \geq p_{\min } \cdot \alpha^{\prime} \cdot \lambda_{G_{s_{0}}}^{k-N_{0}} .
$$


On the other hand we have seen in the proof of Proposition 8.6 that

$$
\sum_{i, j \in G}\left(P G^{k}\right)_{i j} \leq \ell \cdot(\alpha M k)^{m} \cdot \lambda_{G_{s_{0}}}^{k}
$$

where $M$ is the total number of transitional edges. Hence there are constants $M_{1}$ and $M_{2}$ such that

$$
M_{1} \cdot e^{-k \beta} \lambda_{G_{s_{0}}}^{k} \leq e^{-k \beta} \sum_{|\mu|=k} \tau\left(\left\langle x_{\mu}, x_{\mu}\right\rangle\right) \leq M_{2} \cdot e^{-k \beta} \lambda_{G_{s_{0}}}^{k} .
$$

Thus $c_{\tau, \beta}<\infty$ if and only if $h_{X}^{\tau}=\log \lambda_{G_{s_{0}}}<\beta$.

Next we compute the entropies and the phase transitions. We require some notation and terminology. Let $G_{s}$ be a non-zero irreducible component of $G$. Then we can identify the components that communicate with $G_{s}$, say $G_{s_{1}}, \ldots, G_{s_{q}}$ with the understanding that $s_{1}<s_{2}<\cdots<s_{q}$. As "communicating" is transitive we can write

$$
G=\left[\begin{array}{ccc}
* & * & * \\
0 & H_{s} & 0 \\
0 & 0 & *
\end{array}\right] \quad \text { for } \quad H_{s}:=\left[\begin{array}{cccc}
G_{s} & * & \cdots & * \\
0 & G_{s_{1}} & \cdots & * \\
\vdots & \vdots & \cdots & \vdots \\
0 & 0 & \cdots & G_{s_{q}}
\end{array}\right]
$$

up to a permutation of the vertices. We say that $H_{s}$ is the communicating graph on $G_{s}$. We also need the notion of sink for the irreducible component.

Definition 8.8. Let $G$ be a finite graph with irreducible components $G_{1}, \ldots, G_{m}$. A component $G_{s}$ is said to be a sink if there are no paths emitting from $G_{s}$, i.e., if $G_{i j}=0$ for all $i \in G_{s}$ and all $j \notin G_{s}$. Equivalently when $H_{s}=G_{s}$.

The definition of a sink component includes the case where $G_{s}=[0]$. Note that when $G_{s}$ is a sink component then

$$
\sum_{i \in G_{s}} \sum_{j \in G}\left(G^{k}\right)_{i j}=\sum_{i \in G_{s}} \sum_{j \in G_{s}}\left(G^{k}\right)_{i j}=\sum_{i \in G_{s}} \sum_{j \in G_{s}}\left(G_{s}^{k}\right)_{i j} .
$$

Moreover $G_{m}$ will always be a sink component.

Theorem 8.9. Let $G$ be a finite graph with irreducible components $G_{1}, \ldots, G_{m}$. Then

$$
h_{X}^{s}=\max \left\{\log \lambda_{G_{1}}, \ldots, \log \lambda_{G_{m}}\right\} .
$$

If there exists a zero sink component then $h_{X}=0$; otherwise

$$
h_{X}=\min \left\{\log \lambda_{G_{s}} \mid G_{s} \text { is a non-zero sink irreducible component of } G\right\} .
$$

Proof. For the first part, on one hand we can use equation (8.2) to get

$$
\begin{aligned}
h_{X}^{s} & \leq \underset{k}{\limsup } \frac{1}{k} \log \left(\sum_{|\mu|=k}\left\|\left\langle x_{\mu}, x_{\mu}\right\rangle\right\|\right) \\
& =\underset{k}{\limsup } \frac{1}{k} \log \left(\sum_{i, j \in G}\left(G^{k}\right)_{i j}\right)=\max \left\{\log \lambda_{G_{1}}, \ldots, \log \lambda_{G_{m}}\right\} .
\end{aligned}
$$

On the other hand notice that the projections $p_{v}$ are orthogonal and so for any $s=1, \ldots, m$ and $r \in G_{s}$ we get

$$
\begin{aligned}
\left\|\sum_{|\mu|=k}\left\langle x_{\mu}, x_{\mu}\right\rangle\right\| & =\max _{i \in G}\left(\#\left\{x_{\mu}|| \mu \mid=k \text { starting at } v_{i}\right\}\right) \\
& =\max \left\{\sum_{j \in G}\left(G^{k}\right)_{i j} \mid i \in G\right\} \geq \sum_{j \in G_{s}}\left(G_{s}^{k}\right)_{r j} .
\end{aligned}
$$

Applying Proposition 8.2 gives that $h_{X}^{s} \geq \log \lambda_{G_{s}}$ and thus

$$
h_{X}^{s} \geq \max \left\{\log \lambda_{G_{1}}, \ldots, \log \lambda_{G_{m}}\right\} .
$$


For the second part first suppose that $G$ has a zero sink component. Thus its adjacency matrix has a row of zeroes, i.e., there is a vertex $v_{0}$ that emits no edges. If $\tau_{0}$ is the Dirac measure on that vertex then we see that $\sum_{|\mu|=k} \tau_{0}\left(\left\langle x_{\mu}, x_{\mu}\right\rangle\right)=0$ for all $k$, as there are no paths from that vertex. Hence $c_{\tau_{0}, \beta}=1$ and so $\tau_{0} \in \mathrm{T}_{\beta}(A)$ for all $\beta>0$. In particular we get that $h_{X}=0$.

So now suppose that all sink irreducible components are non-zero. Fix an $s_{0}$ such that $\lambda_{G_{s_{0}}}$ corresponds to the minimum of their Perron-Fröbenius. First let a trace $\tau$ supported entirely inside $G_{s_{0}}$ and set $P$ for its corresponding diagonal matrix. Then we have

$$
\sum_{|\mu|=k} \tau\left(\left\langle x_{\mu}, x_{\mu}\right\rangle\right)=\sum_{i, j \in G}\left(P G^{k}\right)_{i j}=\sum_{i \in G_{s_{0}}} \sum_{j \in G}\left(P G^{k}\right)_{i j}=\sum_{i, j \in G_{s_{0}}}\left(P G_{s_{0}}^{k}\right)_{i j} .
$$

Proposition 8.2 (as used in the proof of Theorem 8.3) yields

$$
h_{X}^{\tau}=\log \lambda_{G_{s_{0}}} .
$$

On the other hand let $\tau$ be a trace that is not supported entirely on a sink component. That is, there is a vertex $v_{r}$ that is not in a sink component with $\tau\left(p_{v_{r}}\right)=p_{r} \neq 0$. Hence there is a connecting edge from $v_{r}$ into another irreducible component. That may not be a sink component, but moving inductively we have that there is a sink component $G_{s}$ with which $v_{r}$ communicates. Then Proposition 8.6 gives

$$
h_{X}^{\tau} \geq \log \lambda_{G_{s}} \geq \log \lambda_{G_{s_{0}}} .
$$

As equality holds when $\tau$ is supported entirely on $G_{s_{0}}$ taking the infimum over all $\tau$ gives the required $h_{X}=\log \lambda_{G_{s_{0}}}$.

As a corollary we have the full classification of the equilibrium states and their phase transitions. The parametrizations are weak*-homeomorphisms in this case. We show this in three steps. The following is the analogue of the minimal components in [15].

Definition 8.10. Let $G$ be a finite graph with irreducible components $G_{1}, \ldots, G_{m}$ and let $\lambda>1$. We say that a $G_{s}$ is $\lambda$-maximal if $\lambda_{G_{s}}=\lambda$ and $\lambda_{G_{s}} \geq \lambda_{G_{r}}$ for any $G_{r}$ that is communicated by $G_{s}$.

Proposition 8.11. Let $X$ be a $C^{*}$-correspondence associated to a finite graph with irreducible components $G_{1}, \ldots, G_{m}$. Let

$$
\Lambda:=\left\{\log \lambda_{G_{s}} \mid G_{s} \text { is a } \lambda_{G_{s}} \text {-maximal component }\right\} .
$$

The finite KMS-simplex is constant for any $\beta$ in the half-open half-closed intervals defined by $\Lambda$. Moreover we have an infinite state at $\beta=\log \lambda \geq 0$ if and only if there exists a $\lambda$-maximal component.

Proof. First we show that the possible values for $\tau \in \mathrm{T}(A)$ are $h_{X}^{\tau}<0$ or $h_{X}^{\tau}=\log \lambda$ for some $\log \lambda \in \Lambda$. Suppose that $h_{X}^{\tau}>0$ and by Proposition 8.6 let $G_{s_{0}}$ such that $h_{X}^{\tau}=\log \lambda_{G_{s_{0}}}$. As $h_{X}^{\tau}>0$ then $G_{s_{0}} \neq[0]$. In order to reach contradiction assume that $\log \lambda_{G_{s_{0}}} \notin \Lambda$. Then there exists a $G_{r}$ that is communicated by $G_{s_{0}}$ such that $\lambda_{G_{r}}>\lambda_{G_{s_{0}}}$. But then $G_{r}$ is also communicated by the support of $\tau$ giving the contradiction

$$
h_{X}^{\tau} \geq \log \lambda_{G_{r}}>\log \lambda_{G_{s_{0}}}=h_{X}^{\tau} .
$$

Next we show that $\mathrm{T}_{\beta}(A) \neq \emptyset$ for all $\beta>h_{X}$. Let $G_{s_{0}}$ be the sink component for which $h_{X}=\log \lambda_{G_{s_{0}}}$. Then any trace supported entirely on $G_{s_{0}}$ has entropy equal to $\log \lambda_{G_{s_{0}}}$ and thus is in $\mathrm{T}_{\beta}(A)$ for all $\beta>\log \lambda_{G_{s_{0}}}=h_{X}$.

Now let $\tau \in \mathrm{T}_{\beta}(A)$ with $\beta \in\left(\log \lambda_{1}, \log \lambda_{2}\right]$ and $\log \lambda_{1}, \log \lambda_{2} \in \Lambda$. On the one hand we have $h_{X}^{\tau} \in \Lambda$ from the first paragraph. On the other hand Corollary 8.7 gives that $h_{X}^{\tau}<\beta \leq \log \lambda_{2}$ and so $h_{X}^{\tau} \leq \log \lambda_{1}$. But then $\tau \in \mathrm{T}_{\beta^{\prime}}(A)$ for any other $\beta^{\prime}>\log \lambda_{1}$. Therefore

$$
\mathrm{T}_{\beta}(A)=\mathrm{T}_{\beta^{\prime}}(A) \text { for all } \beta, \beta^{\prime} \in\left(\log \lambda_{1}, \log \lambda_{2}\right] .
$$

For the second part first assume that there is an averaging trace $\tau$ at $\log \lambda>0$. Then $h_{X}^{\tau}=\log \lambda$. Let $G_{s_{1}}, \ldots, G_{s_{q}}$ be all components with which the support of $\tau$ communicates. Then Proposition 8.6 gives a $G_{s_{0}}$ such that

$$
\log \lambda_{G_{s_{0}}}=\max \left\{\log \lambda_{G_{s_{1}}}, \ldots, \log \lambda_{G_{s_{q}}}\right\}=h_{X}^{\tau}=\log \lambda .
$$


Let a $G_{r}$ that is communicated by $G_{s_{0}}$. Then $G_{r}$ is communicated also by the support of $\tau$ so that $G_{r} \in\left\{G_{s_{1}}, \ldots, G_{s_{q}}\right\}$ which implies that

$$
\log \lambda_{G_{r}} \leq \max \left\{\log \lambda_{G_{s_{1}}}, \ldots, \log \lambda_{G_{s_{q}}}\right\}=\lambda_{G_{s_{0}}},
$$

giving that $G_{s_{0}}$ is $\lambda$-maximal.

Conversely suppose that $G_{s_{0}}$ is $\lambda$-maximal for $\lambda \geq 1$. This means that the spectral radius of $H_{s_{0}}$, and thus of $H_{s_{0}}^{t}$, equals $\log \lambda_{G_{s_{0}}}$. A variation of the Perron-Fröbenius Theorem gives an eigenvector $p \geq 0$ of $H_{s_{0}}^{t}$ at $\log \lambda_{G_{s_{0}}}$. Then $p^{\prime}=[0, p, 0]$ is an eigenvector of $G^{t}$ at $\log \lambda_{G_{s_{0}}}$ since

$$
G^{t} \cdot p^{\prime}=\left[\begin{array}{ccc}
* & 0 & 0 \\
* & H_{s_{0}}^{t} & 0 \\
* & 0 & *
\end{array}\right] \cdot\left[\begin{array}{l}
0 \\
p \\
0
\end{array}\right]=\left[\begin{array}{c}
0 \\
H_{s_{0}}^{t} p \\
0
\end{array}\right]=e^{-\lambda_{G_{s_{0}}} \cdot p^{\prime}} .
$$

The $\ell^{1}$-normalization of $p^{\prime}$ defines an averaging trace at $\log \lambda_{G_{s_{0}}}$ and the proof is complete.

Remark 8.12. Our analysis identifies the simplices $\mathrm{T}_{\beta}(A)$ for all $\beta \geq h_{X}$. To this end order the phase transitions in $\Lambda$ by

$$
h_{X}=\log \lambda_{1}<\cdots<\log \lambda_{q}=h_{X}^{s} .
$$

For any such $\log \lambda_{n}$ there are $\lambda_{n}$-maximal components $G_{n, 1}, \ldots, G_{n, k_{n}}$. Notice that no $G_{m, s^{\prime}}$ can be communicated by any $G_{n, s}$ when $m>n$. Set

$$
V_{n}:=\left\{v \in G \mid v \text { communicates with some } G_{n, s}, s=1, \ldots, k_{n}\right\} .
$$

Proposition 8.6 yields

Therefore

$$
h_{X}^{\tau} \leq \log \lambda_{n} \text { iff }\left.\tau\right|_{V_{j}}=0 \text { for all } j>n .
$$

$$
\mathrm{T}_{\beta}(A)=\left\{\tau \in \mathrm{T}(A)|\tau|_{V_{j}}=0 \text { for all } j>n\right\} \text { for all } \beta \in\left(\log \lambda_{G_{s_{n}}}, \log \lambda_{G_{s_{n+1}}}\right] .
$$

In particular there is a finite algorithm to decide if some $\delta_{v}$ is in $\mathrm{T}_{\beta}(A)$ and thus to identify $\mathrm{T}_{\beta}(A)$ :

Step 0. For $v \in G_{s_{0}}$ set $\Lambda_{v}^{(0)}=\lambda_{G_{s_{0}}}$.

Step 1. If $G_{s_{0}+1}$ is communicated by $G_{s_{0}}$ then set $\Lambda_{v}^{(1)}=\left\{\lambda_{G_{s_{0}}}, \lambda_{G_{s_{1}}}\right\}$. Otherwise set $\Lambda_{v}^{(1)}=\Lambda_{v}^{(0)}$.

Step $N$. Suppose that $\Lambda_{v}^{(N-1)}=\left\{\lambda_{G_{s_{0}}}, \ldots, \lambda_{G_{s_{N-1}}}\right\}$. If $G_{s_{N-1}+1}$ is communicated by any of the $G_{s_{0}}, \ldots, G_{s_{N-1}}$ then set $\Lambda_{v}^{(N)}=\Lambda_{v}^{(N-1)} \cup\left\{\lambda_{G_{s_{N-1}+1}}\right\}$. Otherwise set $\Lambda_{v}^{(N)}=\Lambda_{v}^{(N-1)}$.

This algorithm terminates as we have finite components at a step, say $N_{0}$, and set $\lambda_{v}=\max \Lambda_{v}^{\left(N_{0}\right)}$. Then $h_{X}^{\tau}=\log \lambda_{v}$ and so $\delta_{v} \in \mathrm{T}_{\beta}(A)$ whenever $\log \lambda_{v}<\beta$.

Theorem 8.13. Let $X$ be a $C^{*}$-correspondence associated to a finite graph with irreducible components $G_{1}, \ldots, G_{m}$. Let

$$
\Lambda:=\left\{\log \lambda_{G_{s}} \mid G_{s} \text { is } \lambda_{G_{s}} \text {-maximal }\right\}=\left\{h_{X}=\log \lambda_{1}<\cdots<\log \lambda_{q}=h_{X}^{s}\right\}
$$

and let $V_{1}, \ldots, V_{q}$ be the induced vertex sets. Then the phase transitions occur at $\Lambda$ and:

(i) For $\beta \in\left(\log \lambda_{n}, \lambda_{n+1}\right]$ with $\log \lambda_{n}, \log \lambda_{n+1} \in \Lambda$ we have an affine weak ${ }^{*}$-homeomorphism

$$
\Phi:\left\{\tau \in \mathrm{T}(A)|\operatorname{supp} \tau|_{V_{j}}=0 \text { for all } j>n\right\} \rightarrow \mathrm{E}_{\beta}^{\mathrm{fin}}\left(\mathcal{T}_{X}\right) .
$$

(ii) $\operatorname{For} \beta=\log \lambda \in \Lambda$ we have an affine weak*-homeomorphism

$$
\Psi:\left\{\tau \in \mathrm{T}(A) \mid H_{s}^{t} \tau=e^{\beta} \tau \text { for some } G_{s} \text { that is } \lambda \text {-maximal }\right\} \rightarrow \mathrm{E}_{\beta}^{\infty}\left(\mathcal{T}_{X}\right) .
$$

Proof. By Theorem 8.9 we have at least one averaging trace at each of the values in $\Lambda$. The same proof gives the domain of $\Psi$ (which has been shown to be a weak*-homeomorphism). Remark 8.12 shows that the domain of $\Phi$ at $\beta \in\left(\log \lambda_{n}, \log \lambda_{n+1}\right]$ is exactly

$$
\mathrm{T}_{\beta}(A)=\left\{\tau \in \mathrm{T}(A)|\operatorname{supp} \tau|_{V_{j}}=0 \text { for all } j>n\right\},
$$

which is a weak*-closed subset of $\mathrm{T}(A)$. Let

$$
p_{W}:=\sum_{i \in W} p_{v_{i}} \text { for } W:=\left(V_{n+1} \cup \cdots \cup V_{q}\right)^{c} .
$$


For $\tau \in \mathrm{T}(A)$ set $\tau_{W}(\cdot):=\tau\left(p_{W} \cdot p_{W}\right)$ and compute

$$
\sum_{|\mu|=k, s(\mu) \in W} \tau\left(\left\langle x_{\mu}, a x_{\mu}\right\rangle\right)=\sum_{k=0}^{N} e^{-k \beta} \sum_{|\mu|=k} \tau\left(p_{W}\left\langle x_{\mu}, a x_{\mu}\right\rangle p_{W}\right)=\sum_{k=0}^{N} e^{-k \beta} \sum_{|\mu|=k} \tau_{W}\left(\left\langle x_{\mu}, a x_{\mu}\right\rangle\right) .
$$

However by definition $\tau_{W} \in \mathrm{T}_{\beta}(A)$ forcing

$$
\tau\left(\sum_{k=0}^{N} e^{-k \beta} \sum_{|\mu|=k, s(\mu) \in W}\left\langle x_{\mu}, a x_{\mu}\right\rangle\right)=\sum_{k=0}^{N} e^{-k \beta} \sum_{|\mu|=k} \tau_{W}\left(\left\langle x_{\mu}, a x_{\mu}\right\rangle\right)<c_{\tau_{W}, \beta} \cdot\|a\|<\infty
$$

Thus by the Banach-Steinhaus Theorem the sequence $\left(\sum_{k=0}^{N} e^{-k \beta} \sum_{|\mu|=k, s(\mu) \in W}\left\langle x_{\mu}, a x_{\mu}\right\rangle\right)_{N}$ converges, for all $a \in A$. The point here is that $W$ is given by $\beta$, and makes the series to converge.

Suppose that $\tau_{j} \stackrel{\mathrm{w}^{*}}{\longrightarrow} \tau$ in $\mathrm{T}_{\beta}(A)$. Then $\tau_{j}=\left(\tau_{j}\right)_{W}$ and $\tau=\tau_{W}$. We can appeal to the Monotone Convergence Theorem to have that

$$
\begin{aligned}
\lim _{j} \sum_{k=0}^{\infty} e^{-k \beta} \sum_{|\mu|=k} \tau_{j}\left(\left\langle x_{\mu}, a x_{\mu}\right\rangle\right) & =\lim _{j} \sum_{k=0}^{\infty} e^{-k \beta} \sum_{|\mu|=k, s(\mu) \in W} \tau_{j}\left(\left\langle x_{\mu}, a x_{\mu}\right\rangle\right) \\
& =\lim _{j} \tau_{j}\left(\sum_{k=0}^{\infty} e^{-k \beta} \sum_{|\mu|=k, s(\mu) \in W}\left\langle x_{\mu}, a x_{\mu}\right\rangle\right) \\
& =\tau\left(\sum_{k=0}^{N} e^{-k \beta} \sum_{|\mu|=k, s(\mu) \in W}\left\langle x_{\mu}, x_{\mu}\right\rangle\right) \\
& =\sum_{k=0}^{\infty} e^{-k \beta} \sum_{|\mu|=k, s(\mu) \in W} \tau\left(\left\langle x_{\mu}, a x_{\mu}\right\rangle\right) \\
& =\sum_{k=0}^{\infty} e^{-k \beta} \sum_{|\mu|=k} \tau\left(\left\langle x_{\mu}, a x_{\mu}\right\rangle\right) .
\end{aligned}
$$

Thus the parametrization $\Phi$ is weak*-continuous, and hence a weak *-homeomorphism.

We continue with some examples to highlight the methods in the proofs. To this end we will put some effort to compute the predicted values ad-hoc. We start with an example that showcases two points:

(i) It may happen that a trace $\tau$ has $h_{X}^{\tau} \leq 0$ but it may not give an averaging trace at $0=h_{X}^{\tau}$.

(ii) It may be the case that a trace is in $\mathrm{T}_{\beta}(A)$ for all $\beta>0$, so that $\mathcal{T}_{X}$ has finite KMS-states at every $\beta>0$.

Example 8.14. Consider the following graph on two vertices $v$ and $w$ :

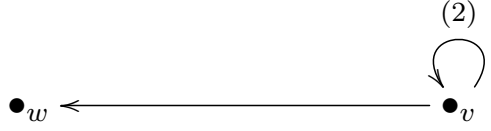

It is immediate that the adjacency matrix satisfies

$$
G=\left[\begin{array}{ll}
2 & 1 \\
0 & 0
\end{array}\right] \quad \text { and } \quad G^{k}=\left[\begin{array}{cc}
2^{k} & 2^{k-1} \\
0 & 0
\end{array}\right]
$$

If $\tau$ is a trace with $\tau\left(p_{v}\right) \neq 0$ then

$$
\sum_{|\mu|=k} \tau\left(\left\langle x_{\mu}, x_{\mu}\right\rangle\right)=\tau\left(p_{v}\right) \cdot\left(2^{k}+2^{k-1}\right)=\tau\left(p_{v}\right) \cdot 3 \cdot 2^{k-1},
$$


and so $h_{X}^{\tau}=\log 2$. On the other hand if $\tau\left(p_{v}\right)=0$ then $\tau=\delta_{w}$ with $c_{\delta_{w}, \beta}=1$ for all $\beta>0$. Moreover we see that $G^{t}$ has an eigenvector $[2 / 3,1 / 3]$ for the eigenvalue 2. Therefore in this example we get

$$
\mathrm{T}_{\beta}(A)=\left\{\begin{array}{ll}
\left\{\delta_{w}\right\} & \text { if } \beta \in(0, \log 2], \\
\mathrm{T}(A) & \text { if } \beta \in(\log 2,+\infty),
\end{array} \quad \text { and } \quad \operatorname{AVT}_{\beta}(A)= \begin{cases}\left\{\frac{2}{3} \delta_{v}+\frac{1}{3} \delta_{w}\right\} & \text { if } \beta=\log 2, \\
\emptyset & \text { otherwise. }\end{cases}\right.
$$

In particular we see that $h_{X}^{\delta_{v}}=\log 2$ but $\delta_{v}$ does not define an averaging trace at $\log 2$ (actually this holds for any trace that is supported on $v$, apart from $p=\frac{2}{3} \delta_{v}+\frac{1}{3} \delta_{w}$ ).

On the other hand we see that $\delta_{w}$ defines a finite KMS-state for all $\beta>0$ given by

$$
\Phi_{\beta}\left(\delta_{w}\right)(f)= \begin{cases}1 & \text { if } f=p_{w} \text { or } f=1, \\ 0 & \text { otherwise }\end{cases}
$$

for all $f \in \mathcal{T}_{X}$. By taking the weak*-limit as $\beta \rightarrow 0$, the KMS-states theory then predicts a gauge-invariant tracial state on $\mathcal{T}_{X}$ of the same form (being constant with respect to $\beta$ ). This can be created directly. Indeed let the representation of $\mathcal{T}_{X}$ on the $\ell^{2}$-path space of $G$, i.e., on the space generated by $\left\{\xi_{\mu} \mid \mu\right.$ is a path in $\left.G\right\}$. Then we get that the vector state

$$
\Phi(f)=\left\langle\xi_{w}, f \xi_{w}\right\rangle \text {, for all } f \in \mathcal{T}_{X},
$$

has the aforementioned form.

The next two examples illustrate the contribution of just the sink irreducible components for getting $h_{X}$, and the contribution of all communicating components for getting a tracial entropy.

Example 8.15. Consider the following graph on three vertices:

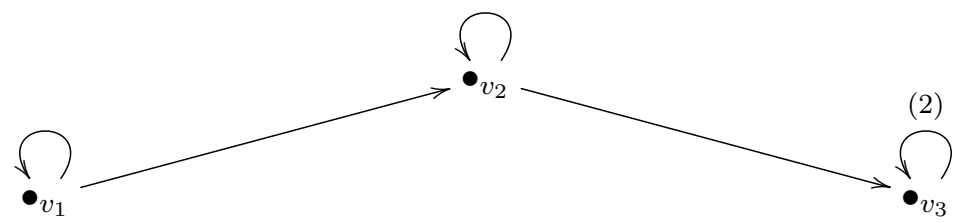

Its adjacency matrix then satisfies

$$
G=\left[\begin{array}{ccc}
1 & 1 & 0 \\
0 & 1 & 1 \\
0 & 0 & 2
\end{array}\right] \quad \text { and } \quad G^{k}=\left[\begin{array}{ccc}
1 & k & a_{k} \\
0 & 1 & b_{k} \\
0 & 0 & 2^{k}
\end{array}\right]
$$

for the sequences

$$
a_{k}= \begin{cases}0 & \text { if } k=1, \\ 1 & \text { if } k=2, \quad \text { and } \quad b_{k}=2^{k+1}-1 \quad \text { for } k \geq 1 . \\ (k-1)+2 a_{k-1} & \text { if } k \geq 3,\end{cases}
$$

On one hand we have that $a_{k} \geq 2^{k-1}$ for $k \geq 2$. On the other hand if $k \geq 2$ then

$$
\begin{aligned}
a_{k} & =(k-1)+2 a_{k-2}=\cdots \\
& =(k-1)+2(k-2)+2^{2}(k-3)+\cdots+2^{k-3}+2^{k-3} a_{2} \\
& \leq k\left(1+2+2^{2}+\cdots+2^{k-3}+2^{k-2}\right)=k \cdot 2^{k-1} .
\end{aligned}
$$

Therefore we have

$$
2^{k-1} \leq k+a_{k} \leq k \cdot 2^{k} \text { and } 2^{k} \leq 1+b_{k} \leq 2^{k+1} .
$$

For a trace $\tau \in \mathrm{T}(A)$ let $p_{i}=\tau\left(p_{v_{i}}\right)$ and set $p_{\max }=\max \left\{p_{1}, p_{2}, p_{3}\right\}$ and $p_{\min }=\min \left\{p_{i} \neq 0 \mid i=\right.$ $1,2,3\}$. Thus we get that

$$
p_{\min } \cdot 2^{k-1} \leq p_{1}\left(k+a_{k}\right)+p_{2}\left(1+b_{k}\right)+p_{3} 2^{k}=\sum_{|\mu|=k} \tau\left(\left\langle x_{\mu}, x_{\mu}\right\rangle\right) \leq p_{\max } \cdot 3 k \cdot 2^{k+1} .
$$

Consequently $c_{\tau, \beta}<\infty$ if and only if $\beta>\log 2$. In particular $h_{X}^{\tau}=\log 2$ for all $\tau \in \mathrm{T}(A)$ and so $h_{X}=\log 2$. We see here that $G$ has one sink irreducible component that contributes to $h_{X}$. 
Moreover $G^{t}$ has two eigenvectors $[0,1 / 2,-1 / 2]$ and $[0,0,1]$ at 1 and 2 , respectively. Thus we have

$$
\mathrm{T}_{\beta}(A)=\left\{\begin{array}{ll}
\emptyset & \text { if } \beta=\log 2, \\
\mathrm{~T}(A) & \text { if } \beta \in(\log 2,+\infty),
\end{array} \text { and } \quad \operatorname{AVT}_{\beta}(A)= \begin{cases}\left\{\delta_{v_{3}}\right\} & \text { if } \beta=\log 2, \\
\emptyset & \text { if } \beta \in(\log 2,+\infty) .\end{cases}\right.
$$

Although $G^{t}$ has a smaller eigenvalue than 2, the corresponding eigenvector is not positive and thus does not contribute to the KMS-simplex.

Example 8.16. Consider the following graph $G$ on three vertices:

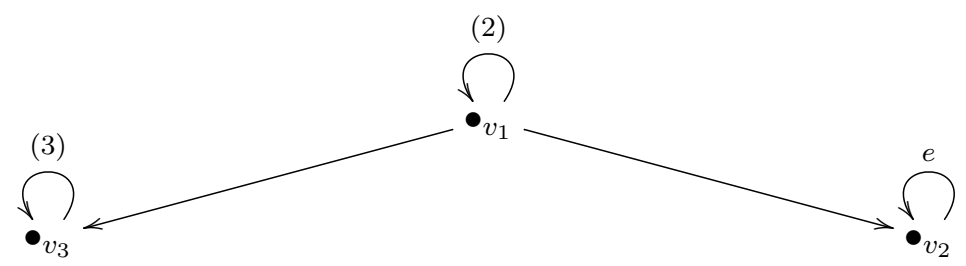

It follows that the adjacency matrix satisfies

$$
G=\left[\begin{array}{lll}
2 & 1 & 1 \\
0 & 1 & 0 \\
0 & 0 & 3
\end{array}\right] \quad \text { and } \quad G^{k}=\left[\begin{array}{ccc}
2^{k} & 2^{k}-1 & 3^{k}-2^{k} \\
0 & 1 & 0 \\
0 & 0 & 3^{k}
\end{array}\right]
$$

Therefore if $\tau \in \mathrm{T}(A)$ with $p_{i}=\tau\left(p_{v_{i}}\right)$ we have that

$$
\sum_{|\mu|=k} \tau\left(\left\langle x_{\mu}, x_{\mu}\right\rangle\right)=p_{1}\left(3^{k}+2^{k}-1\right)+p_{2}+p_{3} 3^{k} .
$$

Set $p_{\max }=\max \left\{p_{1}, p_{2}, p_{3}\right\}$ and $p_{\text {min }}=\min \left\{p_{i} \neq 0 \mid i=1,2,3\right\}$. We then see that if $p_{1} \neq 0$, or if $p_{1}=0$ but $p_{3} \neq 0$ then

$$
p_{\min } 3^{k} \leq \sum_{|\mu|=k} \tau\left(\left\langle x_{\mu}, x_{\mu}\right\rangle\right) \leq p_{\max } 3^{k+1} .
$$

Thus $c_{\tau, \beta}<\infty$ if and only if $\beta>\log 3$. Here we see that $h_{X}^{\tau}=\log 3$ even for $\tau$ with $\tau\left(p_{v_{1}}\right) \neq 0$ and $\tau\left(p_{v_{3}}\right)=0$. Such a state may not be supported on $v_{3}$, e.g., $\tau=\delta_{v_{1}}$, but its support communicates with $v_{3}$ and thus its entropy is affected by it.

On the other hand if $p_{1}=p_{3}=0$ then $\tau=\delta_{v_{2}}$, and so $c_{\delta_{v_{2}}, \beta}<\infty$ if and only if $\beta>0$, in which case $c_{\delta_{2}, \beta}=\left(1-e^{-\beta}\right)^{-1}$. Furthermore $G^{t}$ has three eigenvalues, namely 1,2 and 3 with corresponding $\ell^{1}$-eigenvectors to be $[0,1,0],[1 / 3,1 / 3,-1 / 3]$ and $[0,0,1]$. The averaging traces correspond to positive eigenvectors and so we have that

$$
\mathrm{T}_{\beta}(A)=\left\{\begin{array}{ll}
\left\{\delta_{v_{2}}\right\} & \text { if } \beta \in(0, \log 3], \\
\mathrm{T}(A) & \text { if } \beta \in(\log 3,+\infty),
\end{array} \quad \text { and } \quad \operatorname{AVT}_{\beta}(A)= \begin{cases}\left\{\delta_{v_{3}}\right\} & \text { if } \beta=\log 3 \\
\emptyset & \text { otherwise }\end{cases}\right.
$$

The eigenvector corresponding to the eigevalue 2 does not contribute an averaging trace.

It is worth paying some more attention to $\left\{\delta_{v_{2}}\right\}$. By construction we see that

$$
\begin{aligned}
\delta_{v_{2}}\left(\left\langle x_{f}, p_{v} x_{f}\right\rangle\right) & = \begin{cases}\delta_{v_{2}}\left(p_{s(f)}\right) & \text { if } v=r(f), \\
0 & \text { otherwise, }\end{cases} \\
& = \begin{cases}1 & \text { if } v_{2}=s(f) \text { and } v=r(f), \\
0 & \text { otherwise, }\end{cases} \\
& =\delta_{v_{2}, v} \cdot \delta_{e, f} .
\end{aligned}
$$

Thus $\delta_{v_{2}}$ is an averaging trace at $\beta=0$ since

$$
\sum_{f \in G^{(1)}} \delta_{v_{2}}\left(\left\langle x_{f}, p_{v} x_{f}\right\rangle\right)=\delta_{v_{2}, v}=\delta_{v_{2}}\left(p_{v}\right) .
$$


On the other hand for $\beta \in(0, \log 3)$ we have a single finite KMS-state with the property

$$
\begin{aligned}
\Phi_{\beta}\left(\delta_{v_{2}}\right)\left(L_{\nu_{1}} L_{\nu_{2}}^{*}\right) & =\delta_{\left|\nu_{1}\right|,\left|\nu_{2}\right|}\left(1-e^{-\beta}\right) \sum_{k=0}^{\infty} e^{-k \beta} \sum_{|\mu|=k} \delta_{v_{0}}\left(L_{\mu}^{*} L_{\nu_{2}}^{*} L_{\nu_{1}} L_{\mu}\right) \\
& = \begin{cases}1 & \text { if } \nu_{1}=\nu_{2}=e^{\ell} \text { for some } \ell \in \mathbb{N}, \\
0 & \text { otherwise. }\end{cases}
\end{aligned}
$$

The KMS-states theory predicts the existence of a KMS-state at $\beta=0$ as the weak*-limit of the $\Phi_{\beta}\left(\delta_{v_{2}}\right)$ for $\beta \rightarrow 0$. In particular there exists a tracial state of $\mathrm{C}^{*}(G)$ such that $\Phi=\Phi \circ E$, for the conditional expectation $E$, for which

$$
\Phi\left(L_{\nu_{1}} L_{\nu_{2}}^{*}\right) \begin{cases}1 & \text { if } \nu_{1}=\nu_{2}=e^{\ell} \text { for some } \ell \in \mathbb{N}, \\ 0 & \text { otherwise. }\end{cases}
$$

We will show how this state can be induced directly. To this end let the representation on the infinite path space of $G$. For the infinite path $e^{(\infty)}$ on $v_{2}$ set the state

$$
\Psi(f):=\left\langle\xi_{e(\infty)}, E(f) \xi_{e(\infty)}\right\rangle \text { for all } f \in \mathrm{C}^{*}(G) .
$$

Then a direct computation yields

$$
\begin{aligned}
\Psi\left(L_{\nu_{1}} L_{\nu_{2}}^{*} L_{\nu_{1}^{\prime}} L_{\nu_{2}^{\prime}}^{*}\right) & =\delta_{\left|\nu_{1}\right|+\left|\nu_{1}^{\prime}\right|,\left|\nu_{2}\right|+\left|\nu_{2}^{\prime}\right|}\left\langle L_{\nu_{2}} L_{\nu_{1}}^{*} \xi_{e}(\infty), L_{\nu_{1}^{\prime}} L_{\nu_{2}^{\prime}}^{*} \xi_{e}(\infty)\right\rangle \\
& = \begin{cases}1 & \text { if } \nu_{1}=e^{\ell}, \nu_{2}=e^{k}, \nu_{1}^{\prime}=e^{m}, \nu_{2}^{\prime}=e^{n}, \text { and } \ell+m=k+n, \\
0 & \text { otherwise. }\end{cases}
\end{aligned}
$$

The symmetry on $\nu_{i}, \nu_{i}^{\prime}$ shows that $\Psi$ is tracial. By setting $\nu_{1}^{\prime}=\nu_{2}^{\prime}=\emptyset$, namely the void path, we get that $\Psi$ coincides with $\Phi$. Note that $\Psi$ coincides with the state $\Psi\left(\delta_{v_{0}}\right)$ obtained by the inductive limit process of Theorem 7.1 for the averaging trace $\delta_{v_{0}}$ (even though $\beta=0$ ).

Finally let us give an example with multiple phase transitions. With a small tweak we get a case where the Cuntz-Pimsner algebra has plenty of finite KMS-states.

Example 8.17. Fix a collection of positive integers $\left\{1<a_{1}<\cdots<a_{n}\right\}$ and let the graph $G$ be

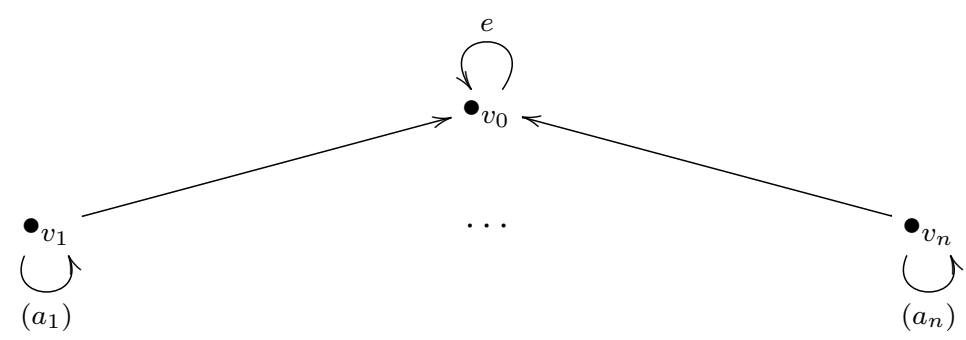

where $\left(a_{j}\right)$ denotes the number of cycles on the vertex $v_{j}$. Its adjacency matrix satisfies

$$
G=\left[\begin{array}{ccccc}
a_{n} & 0 & \cdots & 0 & 1 \\
0 & a_{n-1} & \cdots & 0 & 1 \\
\vdots & \vdots & \cdots & \vdots & \vdots \\
0 & 0 & \cdots & a_{1} & 1 \\
0 & 0 & \cdots & 0 & 1
\end{array}\right] \quad \text { and } \quad G^{k}=\left[\begin{array}{ccccc}
a_{n}^{k} & 0 & \cdots & 0 & \left(a_{n}^{k}-1\right) /\left(a_{n}-1\right) \\
0 & a_{n-1}^{k} & \cdots & 0 & \left(a_{n-1}^{k}-1\right) /\left(a_{n-1}-1\right) \\
\vdots & \vdots & \cdots & \vdots & \vdots \\
0 & 0 & \cdots & 1 & \left(a_{1}^{k}-1\right) /\left(a_{1}-1\right) \\
0 & 0 & \cdots & 0 & 1
\end{array}\right]
$$

Let $\tau$ be a trace on the vertices and set $P$ be the diagonal matrix with $p_{i}=\tau\left(p_{v_{i}}\right)$. Then

$$
\sum_{|\mu|=k} \tau\left(\left\langle x_{\mu}, x_{\mu}\right\rangle\right)=\sum_{i, j \in[n]} p_{i}\left(G^{k}\right)_{i j} .
$$

If $r=\max \left\{i \in[n] \mid p_{i} \neq 0\right\}$ then

$$
\log a_{r}=\lim _{k} \frac{1}{k} \log \left(p_{r} a_{r}^{k-1}\right) \leq h_{X}^{\tau} \leq \lim _{k} \frac{1}{k} \log \left(r^{2} a_{r}^{k+1}\right)=\log a_{r}
$$


and so $h_{X}^{\tau}=\log a_{r}$. Hence any trace supported on $\left\{v_{1}, \ldots, v_{r}\right\}$ defines a state in $\mathrm{E}_{\beta}^{\mathrm{fin}}\left(\mathcal{T}_{X}\right)$ as long as $\beta>\log a_{r}$. Moreover notice that $G^{t}$ admits the vectors $\left\{e_{i} \mid i=1, \ldots, n+1\right\}$ from the o.n. basis of $\mathbb{R}^{n+1}$ as eigenvectors for the eigenvalues $a_{n}, \ldots, a_{2}, a_{1}, 1$. Thus for every $\log a_{i}$ we get that the Dirac measure $\delta_{v_{i}}$ on $v_{i}$ is in $\operatorname{AVT}_{\log a_{i}}(A)$ and so $\mathrm{E}_{\log a_{i}}^{\infty}\left(\mathcal{T}_{X}\right) \neq \emptyset$. Moreover we have that $c_{\delta_{0}, \beta}=1$ for all $\beta>0$. Thus we conclude:

(i) The Dirac measure $\delta_{v_{0}}$ induces a state in $\mathrm{E}_{\beta}^{\text {fin }}\left(\mathcal{T}_{X}\right)$ for all $\beta>0$; therefore we get that $h_{X}=0<\log a_{n}=h_{X}^{s}$.

(ii) If $j>1$ then any convex combination of $\left\{\delta_{v_{j^{\prime}}} \mid j^{\prime}<j\right\}$ induces a state in $\mathrm{E}_{\log a_{j}}^{\mathrm{fin}}\left(\mathcal{T}_{X}\right)$ and $\delta_{v_{j}}$ induces a state in $\mathrm{E}_{\log a_{j}}^{\infty}\left(\mathcal{T}_{X}\right)$.

In this example we have $\operatorname{AVT}_{\beta}(A) \neq \emptyset$ for a finite number of $\beta$, whereas $\mathrm{T}_{\beta}(A) \neq \emptyset$ for all $\beta>0$.

As in Example 8.16 the Dirac measure $\delta_{v_{0}}$ induces a tracial state $\Psi\left(\delta_{v_{0}}\right)$ on $\mathcal{O}_{X}$ and on $\mathcal{T}_{X}$ through the direct limit process of Theorem 7.1, or as the weak*-limit $\Psi\left(\delta_{v_{0}}\right)=\lim _{\beta \rightarrow 0} \Phi_{\beta}\left(\delta_{v_{0}}\right)$.

With a small tweak we can produce a variant $G^{\prime}$ for which $\mathrm{E}_{\beta}^{\text {fin }}\left(\mathcal{O}_{X^{\prime}}\right) \neq \emptyset$ for any $\beta>0$. Here $X^{\prime}$ refers to the graph $\mathrm{C}^{*}$-correspondence related to the graph $G^{\prime}$ given by:

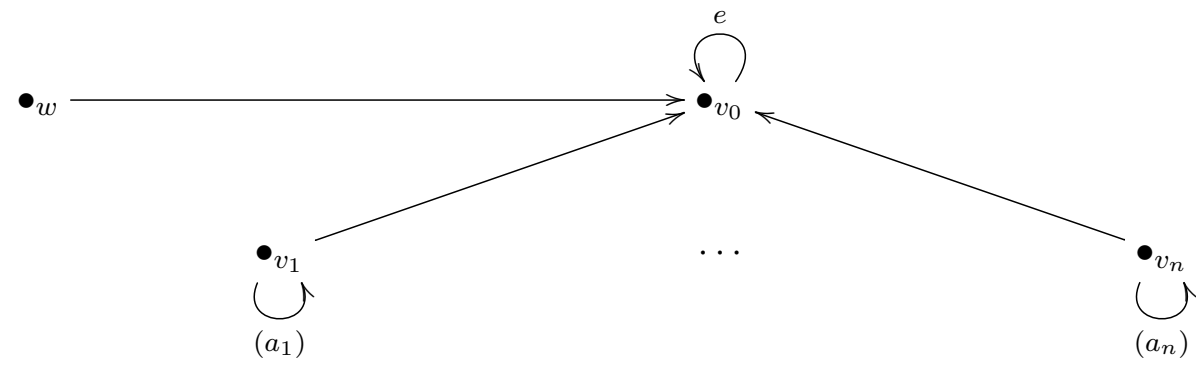

Notice that all the entropies remain the same (there is only one path ending at $v_{0}$ of length $k$ that can be added). If $\tau^{\prime}$ is the Dirac measure on $w$ then $h_{X}^{\tau^{\prime}}=0$ and so $\tau^{\prime} \in \mathrm{T}_{\beta}(A)$ for all $\beta>0$. In light of Theorem 6.4 we have that $\mathcal{O}_{X^{\prime}}$ is the quotient of $\mathcal{T}_{X^{\prime}}$ by $\mathcal{K}\left(\mathcal{F}\left(X^{\prime}\right) J\right)$ for $J=\mathrm{C}^{*}\left(p_{v_{0}}, \ldots, p_{v_{n}}\right)$ and $\left.\tau^{\prime}\right|_{J}=0$. Therefore $\tau^{\prime}$ induces a finite state on $\mathcal{O}_{X^{\prime}}$ for all $\beta>0$.

\section{EXAmples From $[\mathbf{1 5}]$}

We square our analysis with the examples in [15]. The following refer to Examples 6.1-6.7, respectively.

Example 9.1. Let the graph

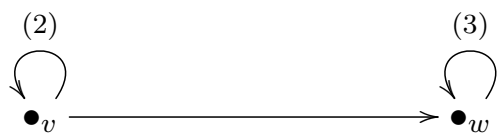

with adjacency matrix

$$
G=\left[\begin{array}{ll}
2 & 1 \\
0 & 3
\end{array}\right]
$$

with respect to the vertices $(w, v)$. The components are $G_{1}=[2]$ and $G_{2}=[3]$ and so $h_{X}^{s}=\log 3$. It has one sink component $G_{2}=[3]$ and thus $h_{X}=\log 3$ as well. We see that $G_{1}$ is not 2-maximal, but $G_{2}$ is 3 -maximal. Hence we have just one phase transition at $\log 3$. Moreover $G_{2}^{t}$ has one eigenvector at 3 given by $\delta_{w}$. By checking the communicating vertices, we have that

$$
h_{X}^{\tau}=\log 3 \text { for all } \tau \in \mathrm{T}(A) .
$$

Therefore for $\mathcal{T}_{X}$ we have

- For $\beta>\log 3$ then $\mathrm{T}_{\beta}(A)=\mathrm{T}(A)=\left\langle\delta_{v}, \delta_{w}\right\rangle$. Thus $\mathrm{E}_{\beta}\left(\mathcal{T}_{X}\right)=\mathrm{E}_{\beta}^{\text {fin }}(A)$ is a simplex of dimension one with extreme points $\Phi\left(\delta_{v}\right)$ and $\Phi\left(\delta_{w}\right)$.

- For $\beta=\log 3$ we have $\mathrm{T}_{\log 3}(A)=\emptyset$ and $\operatorname{AVT}_{\beta}(A)=\left\{\delta_{v}\right\}$. Thus the KMS-simplex has dimension zero with one infinite state induced by $\delta_{v}$.

- For $\beta<\log 3$ we have no KMS-states. 
As $G$ has no sources we have that $\mathcal{O}_{X}=\mathcal{O}(A, X)$. For $\mathcal{O}_{X}$ we thus have

- For $\beta=\log 3$ there is one infinite KMS-state induced by $\delta_{v}$.

- For $\beta \neq \log 3$ we have no KMS-states.

Example 9.2. Let the graph

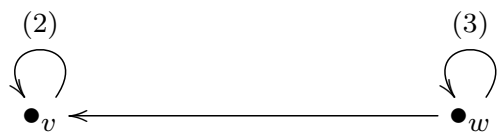

with adjacency matrix

$$
G=\left[\begin{array}{ll}
3 & 1 \\
0 & 2
\end{array}\right]
$$

with respect to the vertices $(w, v)$. The graph has two components $G_{1}=[3]$ and $G_{2}=[2]$. Thus $h_{X}^{s}=\log 3$. The graph has one sink component $G_{2}=[2]$ and so $h_{X}=\log 2$. We see that $G_{1}$ is 3 -maximal and $G_{2}$ is 2-maximal. Hence we have two phase transitions at $\log 2$ and $\log 3$. Moreover $H_{1}^{t}=G^{t}$ has an eigenvector at 3 given by

$$
p=\frac{1}{2}\left(\delta_{w}+\delta_{v}\right)
$$

and $H_{2}^{t}=G_{2}^{t}$ has an eigenvector at 2 given by $\delta_{v}$. By checking the communicating vertices, for $\tau \in \mathrm{T}(A)$ we have

$$
h_{X}^{\tau}= \begin{cases}\log 2 & \text { if } \tau=\delta_{v} \\ \log 3 & \text { otherwise }\end{cases}
$$

Therefore for $\mathcal{T}_{X}$ we have

- For $\beta>\log 3$ we have $\mathrm{T}_{\beta}(A)=\mathrm{T}(A)=\left\langle\delta_{v}, \delta_{w}\right\rangle$. Thus $\mathrm{E}_{\beta}\left(\mathcal{T}_{X}\right)=\mathrm{E}_{\beta}^{\mathrm{fin}}(A)$ is a simplex of dimension one with extreme points $\Phi\left(\delta_{v}\right)$ and $\Phi\left(\delta_{w}\right)$.

- For $\beta=\log 3$ we have $\mathrm{T}_{\log 3}(A)=\left\{\delta_{v}\right\}$ and $\operatorname{AVT}_{\log 3}(A)=\left\{p^{\prime}\right\}$. So the KMS-simplex has dimension one.

- For $\beta \in(\log 2, \log 3)$ we have that $\mathrm{T}_{\beta}(A)=\left\{\delta_{v}\right\}$ and $\operatorname{AVT}_{\log 3}(A)=\emptyset$. Hence we have only one finite KMS-state $\Phi\left(\delta_{v}\right)$.

- For $\beta=\log 2$ we have $\mathrm{T}_{\log 2}(A)=\emptyset$ and $\operatorname{AVT}_{\log 2}(A)=\left\{\delta_{v}\right\}$. Hence we have one infinite KMS-state.

The graph has no sources and so $\mathcal{O}_{X}=\mathcal{O}(A, X)$ inherits the infinite KMS-states:

- For $\beta=\log 3$ there is one KMS-state induced by $p^{\prime}$.

- For $\beta=\log 2$ there is one KMS-state induced by $\delta_{v}$.

- For $\beta \neq \log 2, \log 3$ we have no KMS-states.

Example 9.3. Let the graph

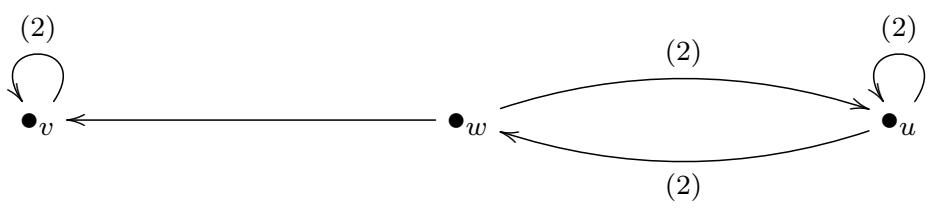

with adjacency matrix and components

$$
G=\left[\begin{array}{lll}
2 & 2 & 0 \\
2 & 0 & 1 \\
0 & 0 & 2
\end{array}\right], \quad G_{1}=\left[\begin{array}{ll}
2 & 2 \\
2 & 0
\end{array}\right], \quad G_{2}=[2],
$$

with respect to the order $(u, w, v)$. We have $\lambda_{G_{1}}=1+\sqrt{5}=: \gamma$ and $\lambda_{G_{2}}=2$. As $\gamma>2$ we have $h_{X}^{s}=\gamma$. We have one sink component, namely $G_{2}$, and so $h_{X}=\log 2$. We see that $G_{1}$ is 
$\gamma$-maximal and $G_{2}$ is 2 -maximal. Hence we have two phase transitions at $\log \gamma$ and $\log 2$. Since $H_{1}=G$ we isolate the eigenvector of $G^{t}$ at $\gamma$ given by

$$
p=\frac{1}{\gamma+1}\left(2 \delta_{u}+(\gamma-2) \delta_{w}+\delta_{v}\right)
$$

while $H_{2}^{t}=G_{2}^{t}$ has an eigenvector at 2 given by $\delta_{v}$. By checking the communicating vertices, for $\tau \in \mathrm{T}(A)$ we have

$$
h_{X}^{\tau}= \begin{cases}\log 2 & \text { if } \tau=\delta_{v} \\ \log \gamma & \text { otherwise }\end{cases}
$$

Therefore for $\mathcal{T}_{X}$ we have

- For $\beta>\log \gamma$ we have $\mathrm{T}_{\beta}(A)=\mathrm{T}(A)=\left\langle\delta_{u}, \delta_{w}, \delta_{v}\right\rangle$. Thus $\mathrm{E}_{\beta}\left(\mathcal{T}_{X}\right)=\mathrm{E}_{\beta}^{\text {fin }}(A)$ is a simplex of dimension two with extreme points $\Phi\left(\delta_{u}\right), \Phi\left(\delta_{w}\right)$ and $\Phi\left(\delta_{v}\right)$.

- For $\beta=\log \gamma$ we have $\mathrm{T}_{\log \gamma}(A)=\left\{\delta_{v}\right\}$ and $\operatorname{AVT}_{\log \gamma}(A)=\{p\}$. So the KMS-simplex has dimension two with one finite state $\Phi\left(\delta_{v}\right)$ and one infinite state $\Psi(p)$.

- For $\beta \in(\log 2, \log \gamma)$ we have $\mathrm{T}_{\beta}(A)=\left\{\delta_{v}\right\}$ and $\operatorname{AVT}_{\beta}(A)=\emptyset$. So the KMS-simplex has only one finite state $\Phi\left(\delta_{v}\right)$.

- For $\beta=\log 2$ we have $\mathrm{T}_{\log 2}(A)=\emptyset$ and $\operatorname{AVT}_{\log 2}(A)=\left\{\delta_{v}\right\}$. So the KMS-simplex has only one infinite state $\Psi\left(\delta_{v}\right)$.

As we have no sources $\mathcal{O}_{X}=\mathcal{O}(A, X)$. Thus $\mathcal{O}_{X}$ inherits just the infinite-type states. That is

- For $\beta=\log \gamma$ it has one KMS-state induced from $p$.

- For $\beta=\log 2$ it has one KMS-state induced from $\delta_{v}$.

- For $\beta \neq \log \gamma, \log 2$ it has no KMS-states.

Example 9.4. Let the graph

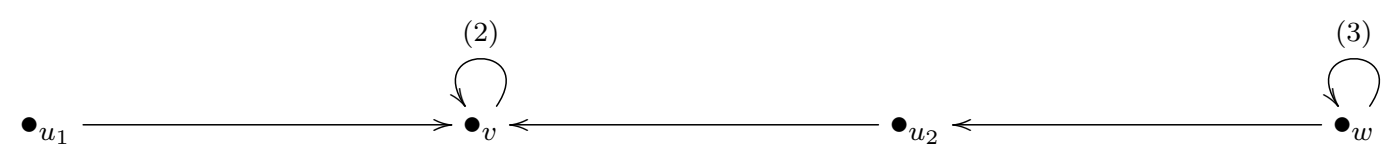

with adjacency matrix and components

$$
G=\left[\begin{array}{llll}
3 & 1 & 0 & 0 \\
0 & 0 & 0 & 1 \\
0 & 0 & 0 & 1 \\
0 & 0 & 0 & 2
\end{array}\right], \quad G_{1}=[3], \quad G_{2}=G_{3}=[0], \quad G_{4}=[2],
$$

with respect to the order $\left(w, u_{2}, u_{1}, v\right)$. We have $\lambda_{G_{1}}=3, \lambda_{G_{2}}=\lambda_{G_{3}}=0$ and $\lambda_{G_{4}}=2$. Therefore $h_{X}^{s}=\log 3$. We have one sink component $G_{4}$ and so $h_{X}=\log 2$. We see that $G_{1}$ is 3 -maximal and $G_{4}$ is 2-maximal. Hence we have two phase transitions at $\log 3$ and $\log 2$. Since $H_{1}=G$ we isolate the eigenvector of $G^{t}$ at 3 given by

$$
p=\frac{1}{5}\left(3 \delta_{w}+\delta_{u_{1}}+\delta_{v}\right),
$$

and the eigenvector of $H_{4}^{t}=G_{4}^{t}$ at 2 given by $\delta_{v}$. By checking the communicating vertices, for $\tau \in \mathrm{T}(A)$ we have

Therefore for $\mathcal{T}_{X}$ we have

$$
h_{X}^{\tau}= \begin{cases}\log 3 & \text { if } w \in \operatorname{supp} \tau \\ \log 2 & \text { otherwise }\end{cases}
$$

- For $\beta>\log 3$ we have $\mathrm{T}_{\beta}(A)=\mathrm{T}(A)$. Thus $\mathrm{E}_{\beta}\left(\mathcal{T}_{X}\right)=\mathrm{E}_{\beta}^{\mathrm{fin}}(A)$ is a simplex of dimension three with extreme points $\Phi\left(\delta_{w}\right), \Phi\left(\delta_{u_{2}}\right), \Phi\left(\delta_{u_{1}}\right)$ and $\Phi\left(\delta_{v}\right)$.

- For $\beta=\log 3$ we have $\mathrm{T}_{\log 3}(A)=\left\langle\delta_{u_{1}}, \delta_{u_{2}}, \delta_{v}\right\rangle$ and $\mathrm{AVT}_{\log 3}(A)=\{p\}$. So the KMS-simplex has dimension 3 , with extreme points at the finite $\Phi\left(\delta_{u_{1}}\right), \Phi\left(\delta_{u_{2}}\right), \Phi\left(\delta_{v}\right)$ and at the infinite $\Psi(p)$. 
- For $\beta \in(\log 2, \log 3)$ we have that $\mathrm{T}_{\beta}(A)=\left\langle\delta_{u_{1}}, \delta_{u_{2}}, \delta_{v}\right\rangle$ and $\operatorname{AVT}_{\beta}(A)=\emptyset$. So the KMSsimplex has dimension 2 with only finite states.

- For $\beta=\log 2$ we have $\mathrm{T}_{\log 2}(A)=\emptyset$ and $\operatorname{AVT}_{\log 2}(A)=\left\{\delta_{v}\right\}$. So the KMS-simplex has zero dimension with one infinite state $\Psi\left(\delta_{v}\right)$.

- For $\beta<\log 2$ we have no KMS-states.

Here we have one source so we need to be careful with the states that are inherited by $\mathcal{O}_{X} \neq$ $\mathcal{O}(A, X)$. For $\mathrm{T}_{\beta}(A)$ we need to intersect with traces that are annihilated at $\left\{w, u_{2}, v\right\}$. On the other hand we inherit the whole $\operatorname{AVT}_{\beta}(A)$ as $A \hookrightarrow \mathcal{O}_{X}$. Hence we have

- For $\beta>\log 3$ we have a single finite state induced by $\delta_{u_{1}}$ and no infinite states.

- For $\beta=\log 3$ we have one finite state induced by $\delta_{u_{1}}$ and one infinite state induced by $p$.

- For $\beta \in(\log 2, \log 3)$ we have a single finite state induced by $\delta_{u_{1}}$ and no infinite states.

- For $\beta=\log 2$ we have a single infinite state induced by $\delta_{u_{1}}$ and no finite states.

- For $\beta<\log 2$ we have no KMS-states.

Example 9.5. Let the graph

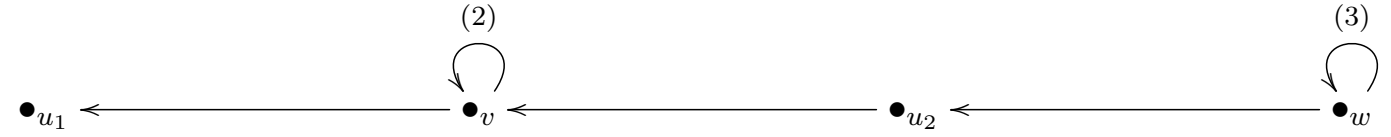

with adjacency matrix and components

$$
G=\left[\begin{array}{llll}
3 & 1 & 0 & 0 \\
0 & 0 & 1 & 0 \\
0 & 0 & 2 & 1 \\
0 & 0 & 0 & 0
\end{array}\right], \quad G_{1}=[3], \quad G_{2}=[0], \quad G_{3}=[2], \quad G_{4}=[0]
$$

with respect to the order $\left(w, u_{2}, v, u_{1}\right)$. We have $\lambda_{G_{1}}=3, \lambda_{G_{2}}=0, \lambda_{G_{3}}=2$ and $\lambda_{G_{4}}=0$. Therefore $h_{X}^{s}=\log 3$. We have one sink component $G_{4}$ and so $h_{X}=0$. We see that $G_{1}$ is 3-maximal, $G_{3}$ is 2 -maximal and $G_{4}$ is 0 -maximal. Hence we have three phase transitions at $0, \log 2$ and $\log 3$. For $G_{1}$ we have $H_{1}^{t}=G^{t}$ and we isolate the eigenvector at 3 given by

$$
p=\frac{1}{16}\left(9 \delta_{w}+3 \delta_{u_{2}}+3 \delta_{u_{1}}+\delta_{v}\right)
$$

For $G_{3}$ we have an eigenvector at 2 from

$$
H_{3}^{t}=\left[\begin{array}{ll}
2 & 0 \\
1 & 1
\end{array}\right] \quad \text { with } \quad p^{\prime}=\frac{1}{2}\left(\delta_{v}+\delta_{u_{1}}\right) .
$$

We also have the eigenvector $\delta_{u_{1}}$ for $H_{4}^{t}=G_{4}^{t}$ at 0 . By checking the communicating vertices, for $\tau \in \mathrm{T}(A)$ we have

$$
h_{X}^{\tau}= \begin{cases}\log 3 & \text { if } w \in \operatorname{supp} \tau \\ 0 & \text { if } \tau=\delta_{u_{1}} \\ \log 2 & \text { otherwise. }\end{cases}
$$

Therefore for $\mathcal{T}_{X}$ we have

- For $\beta>\log 3$ we have $\mathrm{T}_{\beta}(A)=\mathrm{T}(A)$. Thus $\mathrm{E}_{\beta}\left(\mathcal{T}_{X}\right)=\mathrm{E}_{\beta}^{\mathrm{fin}}(A)$ is a simplex of dimension three with extreme points $\Phi\left(\delta_{w}\right), \Phi\left(\delta_{u_{2}}\right), \Phi\left(\delta_{v}\right)$ and $\Phi\left(\delta_{u_{1}}\right)$.

- For $\beta=\log 3$ we have $\mathrm{T}_{\log 3}(A)=\left\langle\delta_{u_{2}}, \delta_{v}, \delta_{u_{1}}\right\rangle$ and $\operatorname{AVT}_{\log 3}(A)=\{p\}$. So the KMS-simplex has dimension 3, with extreme points at the finite $\Phi\left(\delta_{u_{2}}\right), \Phi\left(\delta_{v}\right), \Phi\left(\delta_{u_{1}}\right)$ and at the infinite $\Psi(p)$.

- For $\beta \in(\log 2, \log 3)$ we have that $\mathrm{T}_{\beta}(A)=\left\langle\delta_{u_{2}}, \delta_{v}, \delta_{u_{1}}\right\rangle$ and $\operatorname{AVT}_{\beta}(A)=\emptyset$. So the KMSsimplex has dimension 2 with only finite states.

- For $\beta=\log 2$ we have $\mathrm{T}_{\log 2}(A)=\left\{\delta_{u_{1}}\right\}$ and $\operatorname{AVT}_{\log 2}(A)=\left\{p^{\prime}\right\}$. So the KMS-simplex has dimension one with one extreme finite state $\Phi\left(\delta_{u_{1}}\right)$ and one extreme infinite state $\Psi\left(p^{\prime}\right)$. 
- For $\beta \in(0, \log 2)$ we have $\mathrm{T}_{\beta}(A)=\left\{\delta_{u_{1}}\right\}$ and $\operatorname{AVT}_{\beta}(A)=\emptyset$. So the KMS-simplex has zero dimension with one finite state at $\Phi\left(\delta_{u_{1}}\right)$.

- For $\beta=0$ we get a tracial state induced by $\Phi\left(\delta_{u_{1}}\right)$ (note that $\delta_{u_{1}}$ does not satisfy the averaging condition).

Here we have no sources so $\mathcal{O}_{X}=\mathcal{O}(A, X)$ and inherits only the infinite type states. Hence we have

- For $\beta=\log 3$ we have one infinite state induced by $p$.

- For $\beta=\log 2$ we have one infinite state induced by $p$.

- For $\beta \neq \log 2, \log 3$ we have no KMS-states.

Example 9.6. In this example one source is added to the previous graph to give

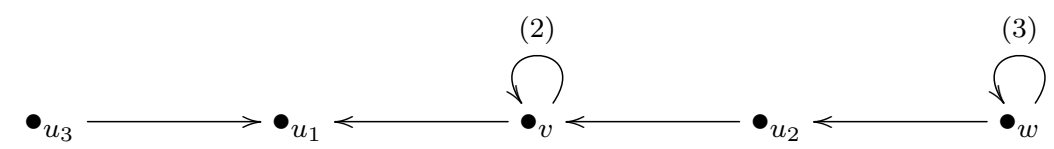

The only change with the previous examplehere KMS-simplex by a finite state. Indeed $u_{3}$ communicates just with $u_{1}$ which is a sink, and so the tracial entropy of $\delta_{u_{3}}$ is zero. As we have a source, then $\mathcal{O}_{X} \neq \mathcal{O}(A, X)$, and in particular $\Phi\left(\delta_{u_{3}}\right)$ descends to a finite state of $\mathcal{O}_{X}$ at every $\beta \in[0,+\infty)$.

Example 9.7. In this example we consider the graph

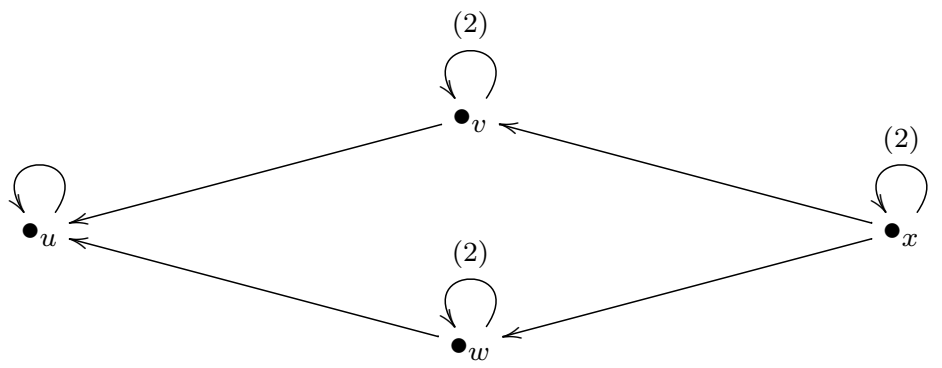

with adjacency matrix and components

$$
G=\left[\begin{array}{llll}
2 & 1 & 1 & 0 \\
0 & 2 & 0 & 1 \\
0 & 0 & 2 & 1 \\
0 & 0 & 0 & 1
\end{array}\right], \quad G_{1}=G_{2}=G_{3}=[2], \quad G_{4}=[1]
$$

with respect to the order $(x, v, w, u)$. We have $\lambda_{G_{1}}=\lambda_{G_{2}}=\lambda_{G_{3}}=2$ and $\lambda_{G_{4}}=1$. Therefore $h_{X}^{s}=\log 2$. We have one sink component $G_{4}$ and so $h_{X}=0$. We see that the components $G_{1}, G_{2}, G_{3}$ are 2-maximal and $G_{4}$ is 1-maximal. Hence we have two phase transitions at 0 and $\log 2$. Here we need to consider the eigenvectors for all $H_{1}^{t}, H_{2}^{t}, H_{3}^{t}, H_{4}^{t}$. For $H_{1}^{t}=G^{t}$ we have two eigenvectors at 2 , namely $[0,1,0,1]$ and $[0,-1,1,0]$, from which we isolate only the positive one. In particular we see that for $G_{2}$ we obtain the "same" eigenvector at 2 from

$$
H_{2}^{t}=\left[\begin{array}{lll}
2 & 0 & 0 \\
0 & 2 & 0 \\
1 & 1 & 1
\end{array}\right] \quad \text { with } \quad p=\frac{1}{2}\left(\delta_{v}+\delta_{u}\right)
$$

For $G_{3}$ we have one eigenvector $p^{\prime}$ at 2 from

$$
H_{3}^{t}=\left[\begin{array}{ll}
2 & 0 \\
1 & 1
\end{array}\right] \quad \text { with } \quad p^{\prime}=\frac{1}{2}\left(\delta_{w}+\delta_{u}\right) .
$$

Finally for $G_{4}$ we have the eigenvector $\delta_{u}$ at 2. By checking the communicating vertices, for a trace $\tau$ we have

$$
h_{X}^{\tau}= \begin{cases}0 & \text { if } \tau=\delta_{u} \\ \log 2 & \text { otherwise }\end{cases}
$$


Therefore for $\mathcal{T}_{X}$ we have

- For $\beta>\log 2$ we have $\mathrm{T}_{\beta}(A)=\mathrm{T}(A)$. Thus $\mathrm{E}_{\beta}\left(\mathcal{T}_{X}\right)=\mathrm{E}_{\beta}^{\mathrm{fin}}(A)$ is a simplex of dimension three with extreme points $\Phi\left(\delta_{x}\right), \Phi\left(\delta_{v}\right), \Phi\left(\delta_{w}\right)$ and $\Phi\left(\delta_{u}\right)$.

- For $\beta=\log 2$ we have $\mathrm{T}_{\log 2}(A)=\left\{\delta_{u}\right\}$ and $\operatorname{AVT}_{\log 2}(A)=\left\langle p, p^{\prime}\right\rangle$. So the KMS-simplex has dimension two, with extreme points at the finite $\Phi\left(\delta_{u}\right)$ and the infinite $\Psi(p)$ and $\Psi\left(p^{\prime}\right)$.

- For $\beta \in(0, \log 2)$ we have that $\mathrm{T}_{\beta}(A)=\left\{\delta_{u}\right\}$ and $\operatorname{AVT}_{\beta}(A)=\emptyset$. So the KMS-simplex has dimension zero with a single finite state $\Phi\left(\delta_{u}\right)$.

- For $\beta=0$ we get a tracial state induced by $\Psi\left(\delta_{u}\right)$.

Here we have no sources so $\mathcal{O}_{X}=\mathcal{O}(A, X)$, and so $\mathcal{O}_{X}$ inherits only the infinite type states. Hence we have

- For $\beta=\log 2$ the KMS-simplex has dimension two with extreme points induced by $\left\{p, p^{\prime}\right\}$.

- For $\beta=0$ we have a tracial state induced by $\delta_{v}$.

- For $\beta \neq 0, \log 2$ we have no KMS-states.

\section{Ground states And $\mathrm{KMS}_{\infty}$-States}

We follow [25] and make a distinction between $\mathrm{KMS}_{\infty}$-states and ground states. The following theorems make that difference clear. The form of the ground states has been identified in [23, Theorem 2.2].

Theorem 10.1. Let $X$ be a $C^{*}$-correspondence of finite rank over $A$. Then there exists an affine weak ${ }^{*}$-homeomoprhism $\tau \mapsto \varphi_{\tau}$ between the states $\tau \in \mathcal{S}(A)$ (resp. the tracial states $\tau \in \mathrm{T}(A)$ ) and the ground states (resp. the $K M S_{\infty}$-states) of $\mathcal{T}_{X}$ such that

$$
\varphi_{\tau}(\pi(a))=\tau(a) \text { for all } a \in A \quad \text { and } \quad \varphi_{\tau}\left(t\left(\xi^{\otimes n}\right) t\left(\eta^{\otimes m}\right)^{*}\right)=0 \text { when } n+m \neq 0 .
$$

Proof. For a state $\tau \in \mathcal{S}(A)$ consider the GNS-representation $\left(H_{\tau}, x_{\tau}, \rho_{\tau}\right)$. Let again $(\rho, v)$ be the induced representation of $\mathcal{T}_{X}$ on $H=\mathcal{F} X \otimes_{\rho_{\tau}} H_{\tau}$ and let $\varphi_{\tau}$ be the vector state given by

$$
\varphi_{\tau}(f):=\left\langle x_{0} \otimes x_{\tau},(\rho \times v)(f) x_{0} \otimes x_{\tau}\right\rangle_{H}=\tau\left(p_{0} f p_{0}\right) .
$$

It is immediate that $\varphi_{\tau}$ satisfies the conditions of the statement. This also shows that the map $\tau \mapsto \varphi_{\tau}$ is injective.

Next we show that equation (10.1) characterizes the ground states for $\tau \in \mathcal{S}(A)$. Then surjectivity follows by noting that if $\varphi$ is a ground state of $\mathcal{T}_{X}$ then $\varphi=\varphi_{\tau}$ for $\tau=\varphi \pi$. Let $\varphi$ be a ground state and let $m \neq 0$. Then the function

$$
r+i s \mapsto \varphi\left(t\left(\xi^{\otimes n}\right) \sigma_{r+i s}\left(t\left(\eta^{\otimes m}\right)^{*}\right)\right)=e^{-i m r} e^{m s} \varphi\left(t\left(\xi^{\otimes n}\right) t\left(\eta^{\otimes m}\right)^{*}\right)
$$

has to be bounded for all $s>0$. This can happen only if $\varphi\left(t\left(\xi^{\otimes n}\right) t\left(\eta^{\otimes m}\right)^{*}\right)=0$. Now if $m=0$ and $n \neq 0$ then we get that $\varphi\left(t\left(\xi^{\otimes n}\right)\right)=0$ by taking adjoints. In any case

$$
\varphi\left(t\left(\xi^{\otimes n}\right) t\left(\eta^{\otimes m}\right)^{*}\right)=0 \text { when } n+m \neq 0 .
$$

Since $\sigma_{z}=$ id on $\pi(a)$ we also get that $\varphi \pi \in \mathcal{S}(A)$ and so $\varphi$ satisfies equation (10.1). Conversely suppose that $\varphi$ satisfies equation (10.1). We have to show that, for any pair

$$
f=t\left(\xi^{\otimes n}\right) t\left(\eta^{\otimes m}\right)^{*} \quad \text { and } \quad g=t\left(\zeta^{\otimes k}\right) t\left(y^{\otimes l}\right)^{*},
$$

the function $r+i s \mapsto \varphi\left(f \sigma_{r+i s}(g)\right)$ is bounded when $t>0$. Indeed we have that

$$
\left|\varphi\left(f \sigma_{r+i s}(g)\right)\right|^{2}=e^{-(k-\ell) 2 s}|\varphi(f g)|^{2} \leq e^{-(k-\ell) 2 s} \varphi\left(f^{*} f\right) \varphi\left(g^{*} g\right) .
$$

This is clearly bounded when $k-l \geq 0$. Now if $k-\ell<0$ then $l>0$ and so

$$
\varphi\left(g^{*} g\right)=\varphi\left(t\left(y^{\otimes \ell}\left\langle\zeta^{\otimes k}, \zeta^{\otimes k}\right\rangle\right) t\left(y^{\otimes \ell}\right)^{*}\right)=0
$$

and thus $\varphi\left(f \sigma_{r+i s}(g)\right)=0$, which completes the proof. 
Now we pass to the $\mathrm{KMS}_{\infty}$-states. Suppose that $\varphi$ is a $\mathrm{KMS}_{\infty}$-state. Due to weak ${ }^{*}$-compactness (and after passing to subsequences), we may choose a sequence $\beta_{j} \uparrow \infty$ such that $\mathrm{w}^{*}-\lim _{j} \varphi_{\tau, \beta_{j}}$ converges to a $\mathrm{KMS}_{\infty}$-state $\varphi$. Then $\left.\varphi\right|_{\pi(A)}$ is tracial and when $n+m \neq 0$ then

$$
\varphi\left(t\left(\xi^{\otimes n}\right) t\left(\eta^{\otimes m}\right)\right)=\lim _{\beta_{j} \rightarrow \infty} e^{-\beta_{j} n} \delta_{n, m} \varphi_{\tau, \beta_{j}}\left(t\left(\eta^{\otimes m}\right)^{*} t\left(\xi^{\otimes n}\right)\right)=0,
$$

so that $\varphi$ satisfies equation (10.1). For surjectivity let $\varphi$ be a $\operatorname{KMS}_{\infty}$-state and set $\tau=\varphi \pi$. Let $\beta_{j} \uparrow \infty$ and without loss of generality assume that $\beta_{j}>h_{X}^{\tau}$ for all $j$. Then we can form $\varphi_{\tau, \beta_{j}} \in \mathrm{E}_{\beta_{j}}^{\mathrm{fin}}\left(\mathcal{T}_{X}\right)$ arising from Theorem 6.1. After passing to a subsequence let $\varphi_{\tau}=\mathrm{w}^{*}-\lim _{j} \varphi_{\tau, \beta_{j}}$. We will show that $\varphi=\varphi_{\tau}$. For $n+m \neq 0$ we have that

$$
\varphi\left(t\left(\xi^{\otimes n}\right) t\left(\eta^{\otimes m}\right)^{*}\right)=0=\varphi_{\tau}\left(t\left(\xi^{\otimes n}\right) t\left(\eta^{\otimes m}\right)^{*}\right) .
$$

Hence it suffices to show that $\varphi_{\tau} \pi=\tau$. Fix a unit decomposition $x=\left\{x_{1}, \ldots, x_{d}\right\}$. Then for $a \in A$ we have

$$
\varphi_{\tau, \beta_{j}}(\pi(a))=c_{\tau, \beta_{j}}^{-1} \tau(a)+c_{\tau, \beta_{j}}^{-1} \sum_{k=1}^{\infty} e^{-k \beta_{j}} \sum_{|\mu|=k} \tau\left(\left\langle x_{\mu}, a x_{\mu}\right\rangle\right) .
$$

Take $\varepsilon>0$ so that $h_{X}^{\tau}+\varepsilon<\beta_{1} \leq \beta_{j}$. Then there exists an $N \in \mathbb{N}$ such that $\sum_{|\mu|=k} \tau\left(\left\langle x_{\mu}, x_{\mu}\right\rangle\right) \leq$ $e^{k\left(h_{X}^{\tau}+\varepsilon\right)}$ for all $k \geq N$. Therefore we get that

$$
\begin{aligned}
1 & \leq c_{\tau, \beta_{j}} \leq 1+\sum_{k=1}^{N-1} e^{-k \beta_{j}} \sum_{|\mu|=k} \tau\left(\left\langle x_{\mu}, x_{\mu}\right\rangle\right)+\sum_{k=N}^{\infty} e^{-k \beta_{j}} e^{k\left(h_{X}^{\tau}+\varepsilon\right)} \\
& =1+e^{N\left(-\beta_{j}+h_{X}^{\tau}+\varepsilon\right)} \frac{1}{1-e^{-\beta_{j}+h_{X}^{\tau}+\varepsilon}}+\sum_{k=1}^{N-1} e^{-k \beta_{j}} \sum_{|\mu|=k} \tau\left(\left\langle x_{\mu}, x_{\mu}\right\rangle\right) .
\end{aligned}
$$

However we have that

$$
\lim _{\beta_{j} \rightarrow \infty}\left[e^{N\left(-\beta_{j}+h_{X}^{\tau}+\varepsilon\right)} \frac{1}{1-e^{-\beta_{j}+h_{X}^{\tau}+\varepsilon}}+\sum_{k=1}^{N-1} e^{-k \beta_{j}} \sum_{|\mu|=k} \tau\left(\left\langle x_{\mu}, x_{\mu}\right\rangle\right)\right]=0
$$

which gives $\lim _{\beta_{j} \rightarrow \infty} c_{\tau, \beta_{j}}=1$. Combining with positivity of $\tau$ we also derive that

$$
\begin{aligned}
\mid \sum_{k=1}^{\infty} e^{-k \beta_{j}} \sum_{|\mu|=k} \tau & \tau\left(\left\langle x_{\mu}, a x_{\mu}\right\rangle\right) \mid \leq \\
& \leq\|a\| \sum_{k=1}^{\infty} e^{-k \beta_{j}} \sum_{|\mu|=k} \tau\left(\left\langle x_{\mu}, x_{\mu}\right\rangle\right) \\
& \leq\|a\|\left[e^{N\left(-\beta_{j}+h_{X}^{\tau}+\varepsilon\right)} \frac{1}{1-e^{-\beta_{j}+h_{X}^{\tau}+\varepsilon}}+\sum_{k=1}^{N-1} e^{-k \beta_{j}} \sum_{|\mu|=k} \tau\left(\left\langle x_{\mu}, x_{\mu}\right\rangle\right)\right] \stackrel{\beta_{j} \rightarrow \infty}{\longrightarrow} 0 .
\end{aligned}
$$

Thus taking limits $\beta_{j} \uparrow \infty$ in equation (10.2) we conclude the required $\varphi(\pi(a))=\tau(a)$.

Finally we have the analogues for the ground states and the $\mathrm{KMS}_{\infty}$-states for $J$-relative CuntzPimsner algebras.

Theorem 10.2. Let $X$ be a $C^{*}$-correspondence of finite rank over $A$. Suppose that $J \subseteq \phi_{X}^{-1}(\mathcal{K} X)$. Then the mapping $\tau \mapsto \varphi_{\tau}$ for

$$
\varphi_{\tau}(\rho(a))=\tau(a) \text { for all } a \in A \quad \text { and } \quad \varphi_{\tau}\left(v\left(\xi^{\otimes n}\right) v\left(\eta^{\otimes m}\right)^{*}\right)=0 \text { when } n+m \neq 0
$$

defines an affine weak*-homeomorphism from the states on $A$ (resp. from the tracial states on $A$ ) that vanish on $J$ onto the ground states of $\mathcal{O}(J, X)$ (resp. onto the $K M S_{\infty}$-states) of $\mathcal{O}(J, X)$.

Acknowledgements. The author would like to thank Marios Bounakis, David Kimsey and Gerasimos Rigopoulos for discussions on links with Physics. The author would like also to thank Sergey Neshveyev for his very helpful remarks and corrections on a preprint of this paper. 


\section{REFERENCES}

[1] J.-B. Bost and A. Connes, Hecke algebras, type III factors and phase transitions with spontaneous symmetry breaking in number theory, Selecta Math. (N.S.) 1:3 (1995), 411-457.

[2] O. Bratteli, G.A. Elliott and A. Kishimoto, The temperature space of a $C^{*}$-dynamical system I, Yokohama Math. J. 28:1-2 (1980), 125-167.

[3] O. Bratteli and D.W. Robinson, Operator algebras and quantum statistical mechanics: $C^{*}$-and $W^{*}$-algebras. Symmetry groups. Decomposition of states, Second Edition, Texts and Monographs in Physics, vol. 1, Springer, New York, 1987.

[4] O. Bratteli and D.W. Robinson, Operator algebras and quantum statistical mechanics: Equilibrium states. Models in quantum statistical mechanics, Second Edition, Texts and Monographs in Physics, vol. 2, Springer, Berlin, 1997.

[5] L.G. Brown, Stable isomorphism of hereditary subalgebras of $C^{*}$-algebras, Pacific J. Math. 71:2 (1977), $335-348$.

[6] F. Combes and H. Zettl, Order structures, traces and weights on Morita equivalent $C^{*}$-algebras, Math. Ann. 265 (1983), 67-81.

[7] J. Cuntz, C. Deninger and M. Laca, $C^{*}$-algebras of Toeplitz type associated with algebraic number fields, Math. Ann. 355:4 (2013), 1383-1423.

[8] J. Cuntz and G.K. Pedersen, Equivalence and traces on periodic $C^{*}$-dynamical systems, J. Funct. Anal. 33 (1979), 135-164.

[9] M. Enomoto, M. Fujii and Y. Watatani, KMS states for gauge action on $\mathcal{O}_{A}$, Math. Japon. 29:4 (1984), 607-619.

[10] D.E. Evans, On $\mathcal{O}_{n}$, Publ. Res. Inst. Math. Sci. 16:3 (1980), 915-927.

[11] R. Exel, Crossed-products by finite index endomorphisms and KMS states, J. Funct. Anal. 199:1 (2003), 153183.

[12] R. Exel, A. an Huef and I. Raeburn, Purely infinite simple $C^{*}$-algebras associated to integer dilation matrices, Indiana Univ. Math. J. 60:3 (2011), 1033-1058.

[13] R. Exel and M. Laca, Partial dynamical systems and the KMS condition, Comm. Math. Phys. 232:2 (2003), $223-277$.

[14] A. an Huef, M. Laca, I. Raeburn and A. Sims, KMS states on the $C^{*}$-algebras of finite graphs, J. Math. Anal. Appl. 405:2 (2013), 388-399.

[15] A. an Huef, M. Laca, I. Raeburn and A. Sims, KMS states on the $C^{*}$-algebras of reducible graphs, Ergodic Theory Dynam. Systems 35:8 (2015), 2535-2558.

[16] M. Izumi, T. Kajiwara and Y. Watatani, KMS states and branched points, Ergodic Theory Dynam. Systems 27:6 (2007), 1887-1918.

[17] T. Kajiwara, Countable bases for Hilbert $C^{*}$-modules and classification of KMS states, Operator Structures and Dynamical Systems, Contemporary Mathematics, 503 (2009), 73-91.

[18] T. Kajiwara and Y. Watatani, KMS-states on finite graph $C^{*}$-algebras, Kyushu J. Math. 67:1 (2013), 83-104.

[19] E.T.A. Kakariadis, A note on the gauge invariant uniqueness theorem for $C^{*}$-correspondences, Israel J. Math. 215:2 (2016), 513-521.

[20] E.T.A. Kakariadis, KMS states on Pimsner algebras associated with $C^{*}$-dynamical systems, J. Funct. Anal. 269:2 (2015), 325-354.

[21] T. Katsura, On $C^{*}$-algebras associated with $C^{*}$-correspondences, J. Funct. Anal. 217:2 (2004), 366-401.

[22] A. Kumjian and J. Renalut, KMS states on $C^{*}$-algebras associated to expansive maps, Proc. Amer. Math. Soc. 134:7 (2006), 2067-2078.

[23] M. Laca and S. Neshveyev, KMS states of quasi-free dynamics on Pimsner algebras, J. Funct. Anal. 211:2 (2004), 457-482.

[24] M. Laca and S. Neshveyev, Type III 1 equilibrium states of the Toeplitz algebra of the affine semigroup over the natural numbers, J. Funct. Anal. 261:1 (2011), 169-187.

[25] M. Laca and I. Raeburn, Phase transition on the Toeplitz algebra of the affine semigroup over the natural numbers, Adv. Math. 225:2 (2010), 643-688.

[26] M. Laca, I. Raeburn and J. Ramagge, Phase transition on Exel crossed products associated to dilation matrices, J. Funct. Anal. 261:12 (2011), 3633-3664.

[27] M. Laca, I. Raeburn, J. Ramagge and M.F. Whittaker, Equilibrium states on the Cuntz-Pimsner algebras of self-similar actions, J. Funct. Anal. 266:11 (2014), 6619-6661.

[28] D. Lind and B. Marcus, An Introduction to Symbolic Dynamics and Coding, Cambridge University Press, Cambridge, 1995.

[29] K. Matsumoto, Y. Watatani and M. Yoshida, KMS states for gauge actions on $C^{*}$-algebras associated with subshifts, Math. Z. 228:3 (1998), 489-509.

[30] S. Neshveyev and E. Størmer, Dynamical entropy in operator algebras, Springer-Verlag (2006).

[31] D. Olesen and G.K. Pedersen, Some $C^{*}$-algebras with a single KMS-state, Math. Scand. 42:1 (1978), $111-118$.

[32] G.K. Pedersen, Measure theory for $C^{*}$-algebras, III, Math. Scand. 25 (1969), 71-91.

[33] M.V. Pimsner, A class of $C^{*}$-algebras generalizing both Cuntz-Krieger algebras and crossed products by $\mathbb{Z}$, Free probability theory (Waterloo, ON, 1995), 189-212, Fields Inst. Commun., 12, Amer. Math. Soc., Providence, RI, 1997. 
[34] C. Pinzari, Y. Watatani and K. Yonetani, KMS States, entropy and the variational principle in full $C^{*}$-dynamical systems, Comm. Math. Phys. 213:2 (2000), 331-379.

[35] M.A. Rieffel, Induced representations of $C^{*}$-algebras, Adv. Math. 13 (1974), 176-257.

[36] D. Robertson, J. Rout and A. Sims, KMS states on generalised Bunce-Deddens algebras and their Toeplitz extensions, Bull. Malaysian Math. Sci. Soc. 41:1 (2015), 123-157.

[37] K. Thomsen, On the $C^{*}$-algebra of a locally injective surjection and its KMS states, Comm. Math. Phys. 302:2 (2011), 403-423.

[38] K. Thomsen, KMS states and conformal measures, Comm. Math. Phys. 316:3 (2012), 615-640.

School of Mathematics, Statistics and Physics, Newcastle University, Newcastle upon Tyne, NE1 7RU, UK

E-mail address: evgenios.kakariadis@ncl.ac.uk 